LPSC14081

UCD-2014-03

\title{
Constraints on and future prospects for Two-Higgs-Doublet Models in light of the LHC Higgs signal
}

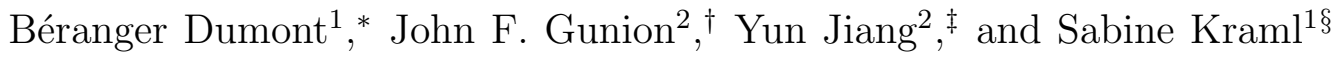 \\ (1) Laboratoire de Physique Subatomique et de Cosmologie, \\ Université Grenoble-Alpes, CNRS/IN2P3, \\ 53 Avenue des Martyrs, F-38026 Grenoble, France and \\ (2) Department of Physics, University of California, Davis, CA 95616, USA
}

\begin{abstract}
We analyze the Two-Higgs-Doublet Models (2HDMs) of Type I and Type II for consistency with the latest measurements of the $\sim 125.5 \mathrm{GeV}$ Higgs-like signal at the LHC. To this end, we perform scans of the 2HDM parameter space taking into account all relevant pre-LHC constraints as well as the most recent limits coming from searches for heavy Higgs-like states at the LHC. The current status of the 2HDMs of Type I and Type II is discussed assuming that the observed $125.5 \mathrm{GeV}$ state is one of the two $C P$-even Higgs bosons, either the lighter $h$ or the heavier $H$. Implications for future experiments, including expectations regarding other lighter or heavier Higgs bosons are given. The possible importance of heavier Higgs bosons feeding the signals for the $125.5 \mathrm{GeV}$ state is also evaluated.

PACS numbers: 12.60.Fr, 14.80.Ec, 14.80.Fd
\end{abstract}

Keywords: Higgs physics, 2-Higgs-Doublet Model, LHC

* dumont@lpsc.in2p3.fr
ifgunion@ucdavis.edu
yunjiang@ucdavis.edu
sabine.kraml@lpsc.in2p3.fr 


\section{INTRODUCTION}

Following the discovery by the ATLAS and CMS experiments at the LHC [1, 2] of a Higgs-like particle, additional measurements of its properties using the full data sets at $\sqrt{s}=7$ and $8 \mathrm{TeV}$ reveal that the observed state with a mass near $125.5 \mathrm{GeV}$ is quite Standard Model (SM)-like [3] [ It is thus clear that models with an extended Higgs sector will be significantly constrained by the data.

In particular, it is interesting to consider the simplest such extensions of the SM, namely Two-Higgs-Doublet Models (2HDMs). For comprehensive reviews see, e.g., [6 8]. These models have attracted a lot of attention recently. A large number of papers [9 18] performed fits of current data for the $125.5 \mathrm{GeV}$ Higgs-like state (as per the status post Moriond 2013) within the context of $2 \mathrm{HDMs}$, and investigated the consequent phenomenology of the other Higgs states present in the models. Among these papers, [10, 11, 13] consider 2HDMs with a conserved $Z_{2}$ symmetry, [15, 16, 19, 20] focus on the case of an aligned 2HDM, [14, 18] investigate the possibility of $C P$-violation in the Higgs sector, and [17] concentrates on the question of the triple-Higgs coupling. The general conditions for the alignment limit, in which the lightest $C P$-even Higgs boson of a 2HDM mimics the Standard Model Higgs, without decoupling the other scalars were studied in [21]. Moreover, the prospects for future LHC running and/or for other future colliders in view of the current data were investigated in [19, 20, 22, 24]. The possibility of $C P$ violation in 2 HDMs also implies an important link of such models to electroweak baryogenesis, a topic that was revisited recently in [25 28].

The present work goes beyond what was done in the above-referenced studies of $2 \mathrm{HDMs}$ in that we provide a very comprehensive and complete analysis of the status of the $C P$ conserving 2HDMs of Type I and Type II, considering both the cases where the observed Higgs particle at the LHC is the lighter $C P$-even state $h$ or the heavier $C P$-even state H. ${ }^{1}$ (The possibility of the heavier $H$ being identified with the $125.5 \mathrm{GeV}$ state was also considered in [11, 13, 16, 23, 24].) In particular, we employ all the latest results for the signal strength measurements from LHC8, include a consistent treatment of feed down (FD) from the production of heavier Higgs states, and discuss the prospects for LHC14.

\footnotetext{
${ }^{1}$ Given that the observed state clearly has $Z Z, W W$ couplings that are not far from SM-like, it cannot be identified with the $A$ which has no $V V$ tree-level couplings. In this paper, we also do not consider cases in which the observed state is a mixture of two or more nearly degenerate $2 \mathrm{HDM}$ states.
} 
In scanning the 2HDM parameter space, we use the parameter set consisting of the physical Higgs masses, $m_{h}, m_{H}, m_{H^{ \pm}}$and $m_{A}$, the $Z_{2}$ soft-breaking parameter $m_{12}$, the $C P$-even Higgs mixing parameter $\alpha$ and the ratio of the two vacuum expectation values $\tan \beta=v_{2} / v_{1}$. We impose all relevant constraints from precision electroweak data, from stability, unitarity and perturbativity of the potential, as well as from $B$ physics and from the direct searches at LEP. For this we closely follow the approach of [29]. Points are retained only when these "preLHC" constraints are all satisfied. Once the preLHC constraints have been applied, we require that the rates for channels involving heavier Higgs bosons all lie below the existing 95\% confidence level (C.L.) limits coming from the LHC.

The next step, and the most important one for this work, is to impose the restrictions on the 2HDM parameter space from the measured Higgs signal. The Higgs measurements are conventionally phrased in terms of "signal strengths", i.e. the ratios to the SM predictions for different production channels, $X$, and different decay modes, $Y$,

$$
\mu_{X}(Y)=\frac{\sigma(X) \mathrm{BR}(Y)}{\sigma_{\mathrm{SM}}(X) \mathrm{BR}_{\mathrm{SM}}(Y)},
$$

where the numerator and denominator are evaluated for the same Higgs mass. The production modes for the Higgs boson considered are ggF (gluon fusion, also denoted as $g g$ ), VBF (vector boson fusion), ttH (associated production with $t \bar{t}$ ) and VH (associated production with a vector boson). The relevant decay modes are those into $Y=\gamma \gamma, V V, b \bar{b}$, and $\tau \tau$ (where $V V \equiv Z Z, W W$ ). In practice, we employ the signal strength likelihoods in the $\mu_{\mathrm{ggF}+\mathrm{ttH}}$ versus $\mu_{\mathrm{VBF}+\mathrm{VH}}$ planes for each of the final states $Y$ as determined in [30], combining all publicly available information from ATLAS and CMS. ${ }^{2}$ The $\left[\mu_{\mathrm{ggF}+\mathrm{ttH}}, \mu_{\mathrm{VBF}+\mathrm{VH}}\right]$ approach has been systematically adopted by the experimental collaborations. It has the advantage of taking into account correlations not accounted for when individual $X \rightarrow H \rightarrow Y$ channels are treated separately. We will require that the Higgs rates for all channels fall within the $95 \%$ C.L. regions in the $\left[\mu_{\mathrm{ggF}+\mathrm{ttH}}, \mu_{\mathrm{VBF}+\mathrm{VH}}\right]$ plane. Points which satisfy the preLHC constraints, the heavy Higgs limits and the Higgs fitting constraints will be labelled

\footnotetext{
${ }^{2}$ Combining VBF and VH is motivated in models where the couplings of the Higgs to $W W$ and to $Z Z$ are scaled equally, as is the case in any $2 \mathrm{HDM}$ because of custodial symmetry. Combining ggF and ttH is more a matter of convenience, partially motivated by the fact that the current LHC measurements do not probe $\mathrm{ggF}$ and $\mathrm{ttH}$ in any given final state at the same time: $H \rightarrow b \bar{b}$ is probed via $\mathrm{ttH}$, not ggF, whereas all the other final states are probed quite precisely via ggF and with much poorer precision via ttH.
} 
as "postLHC8" points.

There is a further issue arising from the fact that there are various ways in which the 125.5 GeV Higgs boson can be produced as a result of feed down from the production of heavier states - in the case of $m_{h}=125.5 \mathrm{GeV}$ this includes the $H, A, H^{ \pm}$[31] while if $m_{H}=125.5 \mathrm{GeV}$ only the $A$ and $H^{ \pm}$can feed the $125.5 \mathrm{GeV}$ signal. If such FD processes occur at a significant rate, the fit to the Higgs measurements using only direct $h$ or $H$ production processes may no longer be valid. For most of our plots, we will show only those postLHC8 points for which the production rate from FD will not distort the fits to the $125.5 \mathrm{GeV}$ resonance. Such points are called "FDOK." The detailed feed down limits employed for a point to be FDOK will be given later. These FD processes may be tested by a variety of means. For example, for the important FD sources of $A \rightarrow Z h$ and $H \rightarrow h h$, the final state mass can be reconstructed and $m_{A}$ and $m_{H}$ will be determined should the rates be significant; see the current limits from CMS in [32. Then, data points lying within the relevant mass windows can be separated off. For the $H \rightarrow h h$ case, the decay products from the second $h$ are visible for most $h$ decays and events with this extra final state "activity" can be separated off. More complicated FD chains will have even more extra particles and constraints that will allow their separation. Feed down must also be considered in the $m_{H}=125.5 \mathrm{GeV}$ case - the process $g g \rightarrow A \rightarrow Z H$ adds events to the $Z H$ final state beyond those from $Z^{*} \rightarrow Z H$; we will comment on this possibility in Section IV.

The rest of the paper is organized as follows. In Section II we give details on the scan ranges and the constraints incorporated. The case of the lighter $C P$-even Higgs $h$ being the observed state near $125.5 \mathrm{GeV}$ is discussed in Section III, and the case of the heavier $C P$ even Higgs $H$ in Section IV] Section V contains our conclusions. Details on the feed down of a heavier Higgs to the $125.5 \mathrm{GeV}$ state are discussed in Appendix A. Appendix B gives details regarding the nondecoupling charged-Higgs contributions to the Higgs- $\gamma \gamma$ coupling and the relationship to wrong-sign Higgs Yukawa couplings for down-type quarks.

\section{PROCEDURAL DETAILS}

In this section we provide some details regarding the parameter scans, the fitting of the signal strengths, and the incorporation of limits related to the Higgs bosons that are heavier than the $125.5 \mathrm{GeV}$ state. 


\begin{tabular}{|c|c|c|c|c|c|}
\hline & Type I and Type II & \multicolumn{2}{|c|}{ Type I } & \multicolumn{2}{c|}{ Type II } \\
\hline Higgs & $C_{V}$ & $C_{U}$ & $C_{D}$ & $C_{U}$ & $C_{D}$ \\
\hline$h$ & $\sin (\beta-\alpha)$ & $\cos \alpha / \sin \beta$ & $\cos \alpha / \sin \beta$ & $\cos \alpha / \sin \beta$ & $-\sin \alpha / \cos \beta$ \\
\hline$H$ & $\cos (\beta-\alpha)$ & $\sin \alpha / \sin \beta$ & $\sin \alpha / \sin \beta$ & $\sin \alpha / \sin \beta$ & $\cos \alpha / \cos \beta$ \\
\hline$A$ & 0 & $\cot \beta$ & $-\cot \beta$ & $\cot \beta$ & $\tan \beta$ \\
\hline
\end{tabular}

TABLE I: Tree-level vector boson couplings $C_{V}(V=W, Z)$ and fermionic couplings $C_{F}(F=U, D)$ normalized to their SM values for the Type I and Type II 2HDMs.

\section{A. Scan ranges and procedures}

As in [29], we employ a modified version of the code 2HDMC [33, 34] for our numerical calculations. All relevant contributions to loop-induced processes are taken into account, in particular those with heavy quarks $(t$ and $b), W^{ \pm}$and $H^{ \pm}$. A number of different input sets can be used in the 2HDMC context. We have chosen to use the "physical basis" in which the inputs are the physical Higgs masses $\left(m_{h}, m_{H}, m_{A}, m_{H^{ \pm}}\right)$, the vacuum expectation value ratio $(\tan \beta)$, and the $C P$-even Higgs mixing angle, $\alpha$, supplemented by $m_{12}^{2}$. The additional parameters $\lambda_{6}$ and $\lambda_{7}$ are assumed to be zero as a result of a $Z_{2}$ symmetry being imposed on the dimension- 4 operators under which $H_{1} \rightarrow H_{1}$ and $H_{2} \rightarrow-H_{2} \cdot m_{12}^{2} \neq 0$ is allowed as a "soft" breaking of the $Z_{2}$ symmetry. With the above inputs, $\lambda_{1,2,3,4,5}$ as well as $m_{11}^{2}$ and $m_{22}^{2}$ are determined (the latter two via the minimization conditions for a minimum of the vacuum) [7]. We scan over the following ranges: ${ }^{3}$

$$
\begin{gathered}
\alpha \in[-\pi / 2,+\pi / 2], \quad \tan \beta \in[0.5,60], \quad m_{12}^{2} \in\left[-(2 \mathrm{TeV})^{2},(2 \mathrm{TeV})^{2}\right], \\
m_{A} \in[5 \mathrm{GeV}, 2 \mathrm{TeV}], \quad m_{H^{ \pm}} \in\left[m^{*}, 2 \mathrm{TeV}\right],
\end{gathered}
$$

where $m^{*}$ is the lowest value of $m_{H^{ \pm}}$allowed by LEP direct production limits and $B$ physics constraints. The LEP limits on the $H^{ \pm}$are satisfied by requiring $m_{H^{ \pm}} \geq 90 \mathrm{GeV}$. The lower bounds from $B$ physics are shown as a function of $\tan \beta$ in Fig. 15 of [8] in the case of the Type II model (roughly $m^{*} \sim 300 \mathrm{GeV}$ in this case) and in Fig. 18 of [8] in the case of the

\footnotetext{
3 The upper and lower bounds on $\tan \beta$ are chosen to ensure that the bottom and top Yukawa couplings, respectively, lie within the perturbative region. Unlike the $Z_{2}$ symmetric $2 \mathrm{HDM}$ which constrains $\tan \beta \lesssim$ 7 [22], high $\tan \beta$ values are allowed when the $Z_{2}$ symmetry is softly broken. A safe upper limit, as adopted here, is $\tan \beta \leq 60$.
} 
Type I model.

The couplings, normalized to their SM values, of the Higgs bosons to vector bosons $\left(C_{V}\right)$ and to up- and down-type fermions $\left(C_{U}\right.$ and $\left.C_{D}\right)$ are functions of $\alpha$ and $\beta$ as given in TableI; see e.g. [6] for details. The Type I and Type II models are distinguished only by the pattern of their fermionic couplings. We note that the range of $\alpha$ employed guarantees that the top quark Yukawa coupling is always positive in our convention. For the most part, in particular for the case of $m_{h} \sim 125.5 \mathrm{GeV}$, one also finds that $\sin (\beta-\alpha)>0$.

For the remaining physical Higgs masses, we consider

$$
\left.\left.m_{h} \in[123 \mathrm{GeV}, 128 \mathrm{GeV}], \quad m_{H} \in\right] 128 \mathrm{GeV}, 2 \mathrm{TeV}\right]
$$

for the case that $h$ is the observed state near $125.5 \mathrm{GeV}$, or

$$
m_{H} \in[123 \mathrm{GeV}, 128 \mathrm{GeV}], \quad m_{h} \in[10 \mathrm{GeV}, 123 \mathrm{GeV}[,
$$

for the case that $H$ is the observed state near $125.5 \mathrm{GeV}$. The window of $125.5 \pm 2.5 \mathrm{GeV}$ is adopted to account for theoretical uncertainties. However, we do not consider the cases where the $A$ and/or the other $C P$-even Higgs are close to $125.5 \mathrm{GeV}$ and possibly contribute to the

observed signal. Thus, for $m_{h} \sim 125.5 \mathrm{GeV}\left(m_{H} \sim 125.5 \mathrm{GeV}\right)$ we require that $m_{H}\left(m_{h}\right)$ and $m_{A}$ not be within the $[123,128] \mathrm{GeV}$ window nor within a $m_{h} \pm 4 \mathrm{GeV}\left(m_{H} \pm 4 \mathrm{GeV}\right)$ mass window where $m_{h}\left(m_{H}\right)$ is the particular mass value generated within the $[123,128] \mathrm{GeV}$ range.

We should note that our scans were performed in a manner that provides adequate point density in all regions of the various plots and predictions of interest. This means that, besides very broad (usually flat) scans in the input parameters mentioned above, we also used scans designed to focus on "hard-to-reach" regions of parameter space of particular interest and importance.

\section{B. Limits imposed by nonobservation of Higgs bosons other than the $125.5 \mathrm{GeV}$ state}

As noted in the Introduction, it is necessary to take into account LHC exclusion limits for Higgs bosons that are heavier than $125.5 \mathrm{GeV}$. In the case that the $h$ is identified with the $\sim 125.5 \mathrm{GeV}$ state the relevant channels are $g g \rightarrow H \rightarrow 4 \ell, 2 \ell 2 \nu, g g \rightarrow H, A \rightarrow \tau \tau$ and 
$g g \rightarrow b \bar{b} H, b \bar{b} A \rightarrow b \bar{b} \tau \tau$ at the LHC. When these limits have been applied the points will be denoted by the phrase " $H / A$ limits." In the case that $m_{H} \sim 125.5 \mathrm{GeV}$ is assumed, the only LHC limits that apply are those on $g g \rightarrow A \rightarrow \tau \tau$ and $g g \rightarrow b \bar{b} A \rightarrow b \bar{b} \tau \tau$. Points that remain acceptable are denoted by " $A$ limits". Direct search limits on the $H^{ \pm}$at the LHC do not impact the parameter space once the $B$ physics limits described below are imposed. In addition, the impact of the CMS search for $H \rightarrow h h$ and $A \rightarrow Z h$ [32] and its relation with FD will be discussed in Sections III B and C.

For $H / A \rightarrow \tau \tau$, we employ the recent CMS limits based on the $8 \mathrm{TeV}$ data [35], which are presented separately for the bbH ( $b \bar{b}$ associated production of the Higgs) and ggF production modes. In taking these limits into account, it will be important to note that in Type II models the coupling of the $H$ and $A$ to down-type fermions can be dramatically enhanced at large $\tan \beta$ compared to the SM expectation and that this enhancement will influence both bbH and ggF production. When $m_{A}$ and $m_{H}$ are within $15 \%$ of one another, which is the approximate resolution in the invariant mass of a pair of $\tau$ leptons, we will add their signals together.

Turning to $H \rightarrow Z Z$, we employ the latest ATLAS and CMS searches for heavy Higgs-like states in the $H \rightarrow Z Z \rightarrow 4 \ell$ channel [36, 37] ${ }^{4}$ and the CMS search in the $H \rightarrow Z Z \rightarrow 2 \ell 2 \nu$ channel [39]. In the context of $2 \mathrm{HDMs}$, there are two important considerations associated with using the limits as presented by the ATLAS and CMS collaborations. First, the $V V$ couplings shown in Table Iimply $\left(C_{V}^{h}\right)^{2}+\left(C_{V}^{H}\right)^{2}=1$ for the coupling strengths relative to the SM Higgs. Thus, if the $h$ is the $125.5 \mathrm{GeV}$ state, the $H$ will have a small coupling to $W, Z$ due to the fact that the $h$ must be very SM-like in order to describe the data at $\sim 125.5 \mathrm{GeV}$, as shown, e.g., in Fig. 10 of [30]. Thus, only ggF production is relevant for $H$. However, only ATLAS presents constraints on a high mass Higgs arising purely from the ggF initial state with the full statistics at $7+8 \mathrm{TeV}$, for $m_{H}>200 \mathrm{GeV}$ [36]. All the other results, i.e. (i) ATLAS $H \rightarrow 4 \ell$ for $m_{H} \in[130,180] \mathrm{GeV}$, (ii) CMS $H \rightarrow 4 \ell$ and (iii) CMS $H \rightarrow 2 \ell 2 \nu$ are implemented in our analysis under the assumption that the experimental search is fully inclusive. The limit is thus rescaled by a factor $\sigma_{H_{\mathrm{SM}}}^{\text {tot }} / \sigma_{g g \rightarrow H_{\mathrm{SM}}}$. Second, the width of the $H$

\footnotetext{
${ }^{4}$ Note that the CMS results on $H \rightarrow Z Z$ are presented after combination of the $4 \ell$ (where $\ell=e, \mu$ ) and $2 \ell 2 \tau$ channels. However, the limit is almost uniquely driven by the $4 \ell$ channels as can be seen from the result based on $H \rightarrow Z Z \rightarrow 2 \ell 2 \tau$ only, which is available as supplementary material on the TWiki page [38.
} 
in the 2HDM can be much smaller than the large SM Higgs widths assumed in the ATLAS and CMS analyses. We correct for the width difference by rescaling the observed limits on $\sigma \times$ BR by the factor $f=\sqrt{\frac{\Gamma_{H}^{2}+(4 \mathrm{GeV})^{2}}{\Gamma_{H_{\mathrm{SM}}}^{2}+(4 \mathrm{GeV})^{2}}}$, where $4 \mathrm{GeV}$ is the experimental resolution in the $4 \ell$ final state [40].

\section{Constraints from the signal strength measurements at $125.5 \mathrm{GeV}$}

For each scan point that passes the constraints explained above, we compute the predictions for $\mu_{\mathrm{ggF}+\mathrm{ttH}}(Y)$ and $\mu_{\mathrm{VBF}+\mathrm{VH}}(Y)$ for the main decay modes $Y(\gamma \gamma, V V, b \bar{b}$, and $\tau \tau)$ in terms of the reduced couplings $C_{U}, C_{D}$, and $C_{V}$, see Table II. To this end, we also need the loop-induced $\gamma \gamma$ and $g g$ couplings of the Higgs boson with mass around $125.5 \mathrm{GeV}$; for these we employ the full 1-loop amplitudes in 2HDMC (including the contribution from the charged Higgs bosons in the $\gamma \gamma$ case), where SM contributions are scaled according to the values of $C_{U}, C_{D}$ and $C_{V}$.

To combine the information provided by ATLAS, CMS and the Tevatron experiments on the $\gamma \gamma, Z Z^{(*)}, W W^{(*)}, b \bar{b}$ and $\tau \tau$ final states including the error correlations among the $(\mathrm{VBF}+\mathrm{VH})$ and $(\mathrm{ggF}+\mathrm{ttH})$ production modes, we follow the approach of [30]. Concretely, we fit the likelihood from the $68 \%$ C.L. contour provided by the experiments for each decay mode $Y$ in the $\mu_{\mathrm{ggF}+\mathrm{ttH}}(Y)$ versus $\mu_{\mathrm{VBF}+\mathrm{VH}}(Y)$ plane, using a Gaussian approximation. For each experiment, $-2 \log L_{Y}=\chi_{Y}^{2}$ can then be expressed as

$$
\begin{aligned}
\chi_{Y}^{2} & =\left(\boldsymbol{\mu}_{Y}-\hat{\boldsymbol{\mu}}_{Y}\right)^{T}\left(\begin{array}{cc}
\sigma_{\mathrm{ggF}, Y}^{2} & \rho_{Y} \sigma_{\mathrm{ggF}, Y} \sigma_{\mathrm{VBF}, Y} \\
\rho_{Y} \sigma_{\mathrm{ggF}, Y} \sigma_{\mathrm{VBF}, Y} & \sigma_{\mathrm{VBF}, Y}^{2}
\end{array}\right)^{-1}\left(\boldsymbol{\mu}_{Y}-\hat{\boldsymbol{\mu}}_{Y}\right) \\
& =\left(\boldsymbol{\mu}_{Y}-\hat{\boldsymbol{\mu}}_{Y}\right)^{T}\left(\begin{array}{cc}
a_{Y} & b_{Y} \\
b_{Y} & c_{Y}
\end{array}\right)\left(\boldsymbol{\mu}_{Y}-\hat{\boldsymbol{\mu}}_{Y}\right) \\
& =a_{Y}\left(\mu_{\mathrm{ggF}, Y}-\hat{\mu}_{\mathrm{ggF}, Y}\right)^{2}+2 b_{Y}\left(\mu_{\mathrm{ggF}, Y}-\hat{\mu}_{\mathrm{ggF}, Y}\right)\left(\mu_{\mathrm{VBF}, Y}-\hat{\mu}_{\mathrm{VBF}, Y}\right)+c_{Y}\left(\mu_{\mathrm{VBF}, Y}-\hat{\mu}_{\mathrm{VBF}, Y}\right)^{2},
\end{aligned}
$$

where the indices $\mathrm{ggF}$ and $\mathrm{VBF}$ stand for $(\mathrm{ggF}+\mathrm{ttH})$ and $(\mathrm{VBF}+\mathrm{VH})$, respectively, and $\hat{\mu}_{\mathrm{ggF}, Y}$ and $\hat{\mu}_{\mathrm{VBF}, Y}$ denote the best-fit points obtained from the measurements [30]. The twodimensional (2D) covariance matrix is explicitly shown in the first line of Eq. (5), with $\rho_{Y}$ corresponding to the correlation between the measurement of $(\mathrm{ggF}+\mathrm{ttH})$ and $(\mathrm{VBF}+\mathrm{VH})$. From a digitized version of the $68 \%$ C.L. contour, it is possible to fit simultaneously the 
parameters $a_{Y}, b_{Y}, c_{Y}, \hat{\mu}_{\mathrm{ggF}, Y}$ and $\hat{\mu}_{\mathrm{VBF}, Y}$. A combination of ATLAS and CMS can then be made for each decay mode $Y$ and expressed again in terms of $a_{Y}, b_{Y}, c_{Y}, \hat{\mu}_{\mathrm{ggF}, Y}$ and $\hat{\mu}_{\mathrm{VBF}, Y}$. Adding up the individual $\chi_{Y}^{2}$, we thus obtain a "combined likelihood," which can be used in a simple, generic way to constrain nonstandard Higgs sectors and new contributions to the loop-induced processes, provided they have the same Lagrangian structure as the SM. In this paper, for each scan point that passes the constraints of Sections IIA and IIB, we demand that each $\chi_{Y}^{2}$ (for ATLAS and CMS combined) be smaller than 6.18, which corresponds to 95\% C.L. in two dimensions. These points are labelled as "postLHC8." 


\section{III. $m_{h} \sim 125.5 \mathrm{GeV}$ SCENARIOS}

In this section we focus on the case that the observed $\sim 125.5 \mathrm{GeV}$ state is the $h$.

\section{A. Current constraints}

To cover the case $m_{h}=125.5 \pm 2.5 \mathrm{GeV}$, we scan over $m_{H}, m_{A}$ and $m_{H^{ \pm}}$as discussed earlier in section III. As regards Yukawa couplings, the scan range of $|\alpha| \leq \pi / 2$ implies that $C_{U}^{h}=C_{D}^{h}>0$ for Type I, whereas for Type II $C_{D}^{h}<0$ is possible when $\sin \alpha>0$. Note that with this scanning range for $\alpha, \sin (\beta-\alpha)<0$ is in principle possible, but is excluded by LHC data in the case of $m_{h}=125.5 \mathrm{GeV}$. Thus, for both Type I and Type II models, $C_{V}^{h}$ and $C_{U}^{h}$ are always positive.

As regards the possibilities for $\sin \alpha$, which determines the sign of $C_{D}^{h}$ in Type II, there are important constraints from perturbativity of the quartic Higgs couplings (even in the case of the Type I model). Often the strongest constraint is associated with the $\lambda_{A A A A}$ quartic coupling of four $A$ Higgs bosons. Figure 1 shows the values of $\lambda_{A A A A}$ that arise after the preLHC conditions listed in the Introduction are satisfied. From the figure we see that perturbativity of this quartic coupling creates a boundary of maximal $\sin (\beta-\alpha)$ values for $\sin \alpha>0$ and of minimal $\sin (\beta-\alpha)$ values for $\sin \alpha \lesssim-0.3$. The other boundaries arise as a result of constraining other quartic couplings to their perturbative domain. Note in particular that in both Type I and Type II the maximal value of $\sin (\beta-\alpha)$ decreases as $\sin \alpha$ increases starting from $\sin \alpha \sim 0$, whereas $\sin (\beta-\alpha) \sim 1$ is possible for a broad range of $\sin \alpha<0$ values. This will impact many phenomenological results. In particular, even though the $g g \rightarrow h$ fusion production rate is insensitive (not very sensitive) to the sign of $\sin \alpha$ for Type I (Type II), the $\gamma \gamma$ partial width is - as $\sin (\beta-\alpha)$ declines, the $W$-loop contribution to the $h \gamma \gamma$ coupling decreases, resulting in a decrease in $\operatorname{BR}(h \rightarrow \gamma \gamma)$. Hence, the rate for $g g \rightarrow h \rightarrow \gamma \gamma$ quickly falls below the level acceptable for LHC precision Higgs results. This is also the case for $H \rightarrow W W^{*}$ originating from VBF or VH: while probed with poorer precision, the $\sin (\beta-\alpha)$ factor associated with the $H V V$ vertex is present in both production and decay.

Let us now turn to the constraints on the model parameter space that originate from requiring that the signal strengths of the observed Higgs are matched at 95\% C.L. in each 

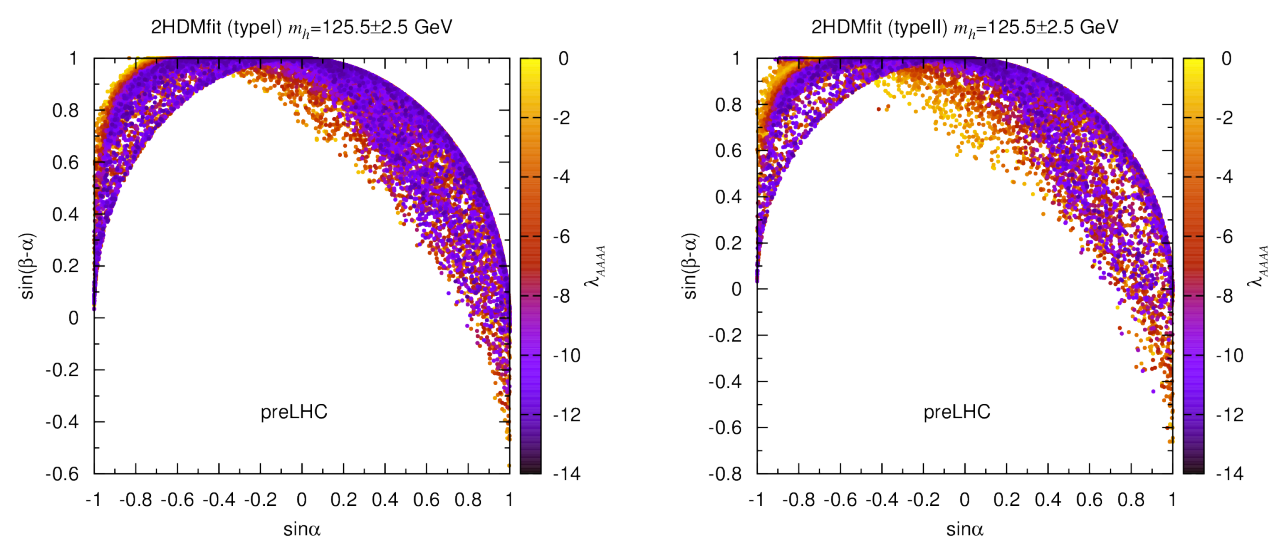

FIG. 1: Values for the quartic coupling $\lambda_{A A A A}$ for 2HDMs of Type I (left) and Type II (right) in the $\sin (\beta-\alpha)$ versus $\sin \alpha$ plane for $m_{h} \sim 125.5 \mathrm{GeV}$ shown as "temperature plots". For all points, the full set of preLHC constraints is satisfied, including all quartic couplings having absolute values below $4 \pi$.

final state. We begin with the plots of Fig. 2 showing points in the $\cos (\beta-\alpha)$ vs. $\tan \beta$ plane. Recall that if the $h$ is SM-like, then $\sin (\beta-\alpha)$ will be large and $\cos (\beta-\alpha)$ will be constrained to smaller values. The color scheme is the following:

- grey points are those that survive the preLHC constraints;

- green points are those for which all LHC limits related to the heavier Higgs bosons are obeyed at 95\% C.L. — we employ the label "H/A limits" for such points;

- blue points are those for which, in addition, the $h$ predictions fall within the 95\% C.L. regions in the $\left[\mu_{\mathrm{ggF}+\mathrm{ttH}}, \mu_{\mathrm{VBF}+\mathrm{VH}}\right]$ plane for all final state channels $(\gamma \gamma, V V, b \bar{b}$, and $\tau \tau)$ - we term points at this level postLHC8 - and in addition the effects of feed down on the 95\% C.L. ellipses is small, which we call "postLHC8-FDOK."

We will discuss FD in more detail later, giving the precise criteria required for a point to be "FDOK". For now, let us note the following features of the plots. Looking at the blue points that survive at the postLHC8-FDOK level, we observe that for $m_{h} \sim 125.5 \mathrm{GeV}$ in Type I models $|\cos (\beta-\alpha)|$ cannot be too large, especially if $\tan \beta \sim 1$. In the Type II models, either $|\cos (\beta-\alpha)|$ can be quite close to 0 or it can fall in a second branch where fairly large positive $\cos (\beta-\alpha) \gtrsim 0.3$ is allowed if $\tan \beta \lesssim 7$. It turns out that this branch is associated with $\sin (\beta+\alpha) \approx 1$ and $\sin \alpha>0$ [for which the $b$-quark Yukawa coupling has 

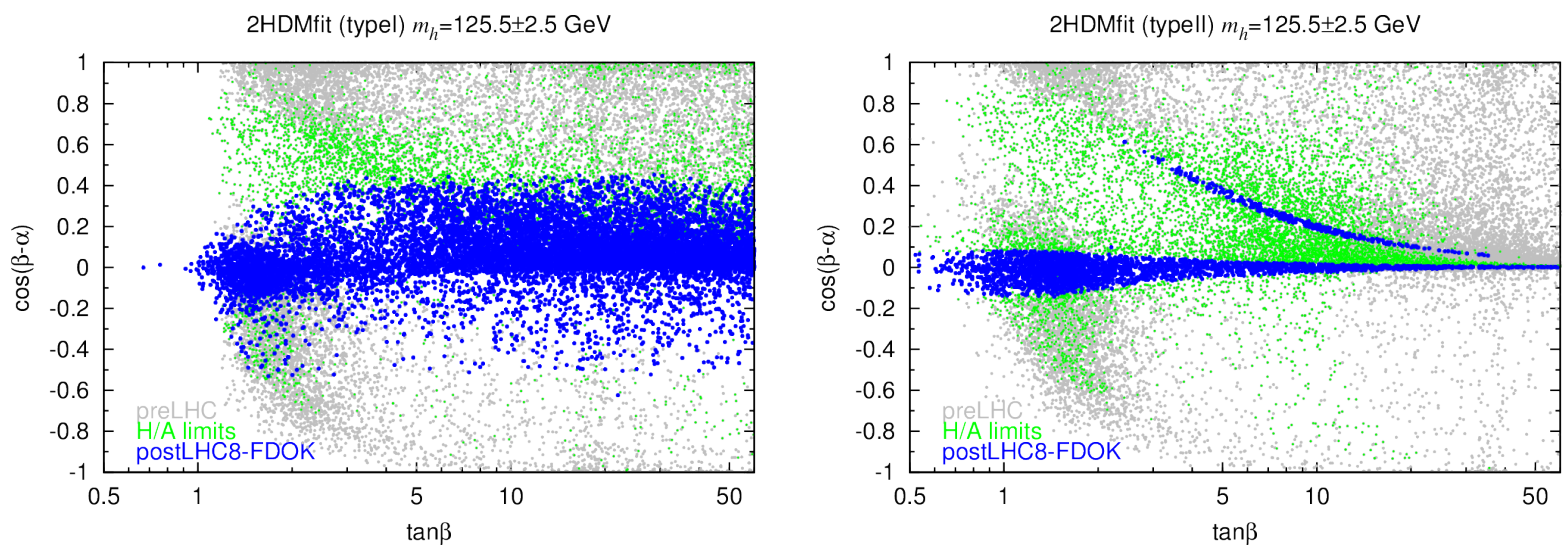

FIG. 2: Constraints in the $\cos (\beta-\alpha)$ versus $\tan \beta$ plane for $m_{h} \sim 125.5 \mathrm{GeV}$. Grey points satisfy preLHC constraints, while green points satisfy in addition the LHC limits on $H$ and $A$ production. Blue points fall moreover within the $7+8 \mathrm{TeV} 95 \%$ C.L. ellipses in the $\left[\mu_{\mathrm{ggF}+\mathrm{ttH}}(Y), \mu_{\mathrm{VBF}+\mathrm{VH}}(Y)\right]$ plane for each of the final states considered $(Y=\gamma \gamma, V V, b \bar{b}, \tau \tau)$, and the amount of FD from $H$ or $A$ production is small.

the opposite sign relative to the $\sin (\beta-\alpha) \rightarrow 1$ limit]. This "wrong-sign" Yukawa coupling, $C_{D}^{h} \sim-1$ is the focus of [41].

Insight into the underlying couplings, $C_{V}^{h}, C_{U}^{h}$ and $C_{D}^{h}$ is provided by Fig. 3. There, we see that for both Type I and Type II, $C_{V}^{h} \sim+1$ is required for a decent fit to the Higgs data. Further, $C_{U}^{h} \sim+1$ is needed in order to describe the observed $\gamma \gamma$ final state rates (i.e. a SM-like cancellation between the $W$ and $t$ loops contributing to the $h \gamma \gamma$ coupling is required). In Type I, $C_{D}^{h}=C_{U}^{h}$ and therefore both must also be close to +1 . However, this is not required in the Type II models. In fact, the second branch apparent in Fig. 2 corresponds to the $C_{D}^{h} \sim-1$ region of the right-hand Type II plot of Fig. 3 - note that the magnitude, $\left|C_{D}^{h}\right| \sim 1$, is approximately fixed by the need for acceptable fits to the $b \bar{b}$ and $\tau \tau$ final state rates.

Also of interest for the $m_{h} \sim 125.5 \mathrm{GeV}$ case is the range of the heavy Higgs masses as a function of $\cos (\beta-\alpha)$, shown in Fig. 4. Clearly, once $m_{A}$ is above about $800 \mathrm{GeV}$ we are deep into the small $|\cos (\beta-\alpha)|$ decoupling region, whereas for masses below $\sim 800 \mathrm{GeV}$ there is considerable spread in the allowed $|\cos (\beta-\alpha)|$ values, in particular in Type I. Thus, if an $A$ (or $H$ or $H^{ \pm}$) is found above $\sim 800 \mathrm{GeV}$, the $2 \mathrm{HDM}$ require that the $h$ is very SMlike, but if $m_{A}$ is found to be lower in mass, then the $h$ need not be so SM-like. Conversely, 

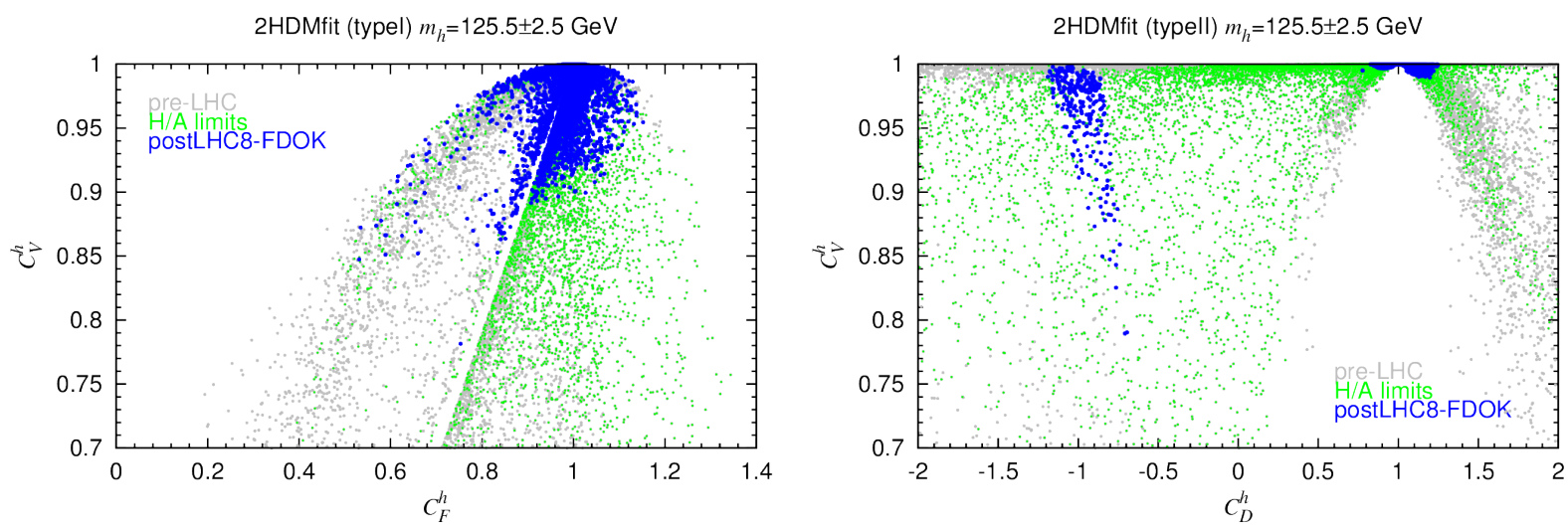

FIG. 3: $C_{V}$ versus $C_{F}=C_{U}=C_{D}$ for Type I (left) and $C_{V}$ versus $C_{D}$ for Type II (right). Color scheme as in Fig. 2. We have restricted the plots to $C_{V} \geq 0.7$ so as to most clearly display the postLHC8-FDOK points.
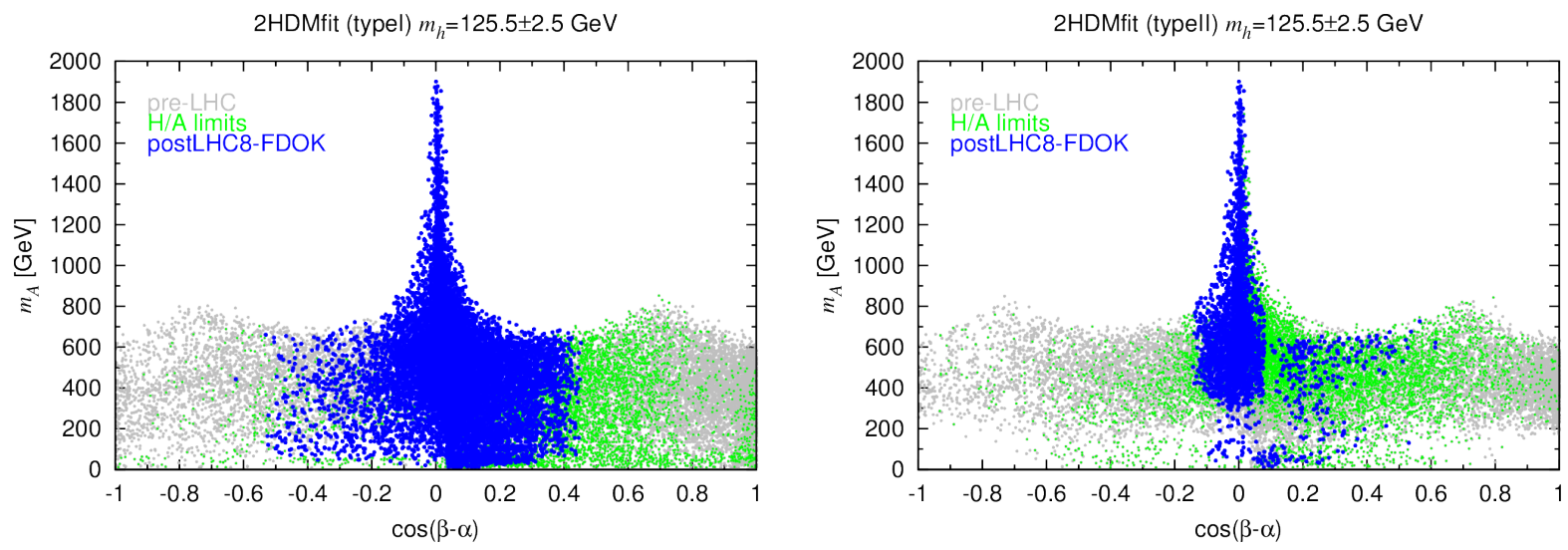

FIG. 4: Constraints in the $m_{A}$ versus $\cos (\beta-\alpha)$ plane for $m_{h} \sim 125.5 \mathrm{GeV}$. Color scheme as in Fig. 2. The corresponding results in the $m_{H}$ vs. $\cos (\beta-\alpha)$ and $m_{H^{ \pm}}$vs. $\cos (\beta-\alpha)$ planes give essentially the same picture as for $m_{A}$, except that $m_{H}$ and $m_{H^{ \pm}}$do not go below $125 \mathrm{GeV}$ and $100 \mathrm{GeV}(300 \mathrm{GeV})$ for Type I (Type II), respectively.

if the $h$ is found to have very SM-like $V V$ coupling, i.e. if $|\cos (\beta-\alpha)| \approx 0$, then $m_{A}, m_{H}$ and $m_{H^{ \pm}}$could each take a large range of values. The larger $\cos (\beta-\alpha)>0$ points in the Type II case are the same as the $C_{D}^{h} \sim-1$ points of Fig. 3. That they cannot occur at high $m_{H}$ is associated with nondecoupling perturbativity limits, see [41].

To illuminate the precision with which individual channels are being fit, we show in Fig. 5 the signal strengths for $\mu_{g g}^{h}(Z Z)$ vs. $\mu_{g g}^{h}(\gamma \gamma)$ (upper row) as well as for $\mu_{\mathrm{VBF}}^{h}(\gamma \gamma)$ vs. $\mu_{g g}^{h}(\gamma \gamma)$ 

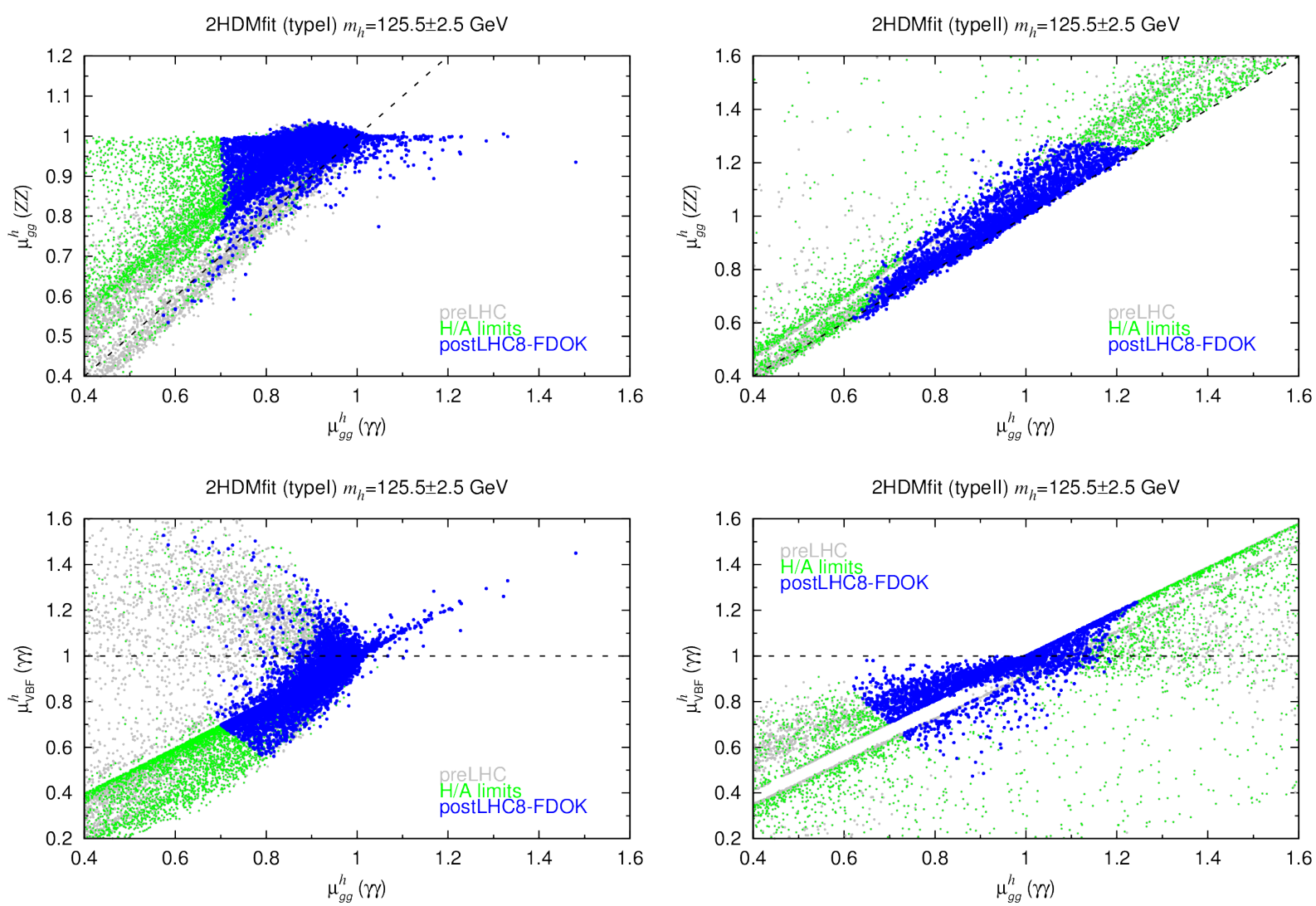

FIG. 5: Correlations of signal strengths for the $m_{h} \sim 125.5 \mathrm{GeV}$ scenario following the color scheme of Fig. 2] in the upper row $\mu_{g g}^{h}(Z Z)$ vs. $\mu_{g g}^{h}(\gamma \gamma)$, in the lower row $\mu_{\mathrm{VBF}}^{h}(\gamma \gamma)$ vs. $\mu_{g g}^{h}(\gamma \gamma)$.

(lower row). From this figure, it is apparent that requiring the $\mu$ values to lie within $\pm 10 \%$ of unity would have a strong impact. Even $\pm 20 \%$ measurements will remove many parameter choices. Note also that with sufficiently precise measurements of $\mu_{g g}^{h}(Z Z)$ and $\mu_{g g}^{h}(\gamma \gamma)$ there is a chance to distinguish Type I from Type II models; for most of the blue points if $\mu_{g g}^{h}(\gamma \gamma)$ is $>1$, then $\mu_{g g}^{h}(Z Z) / \mu_{g g}^{h}(\gamma \gamma)<1$ for Type I, whereas for Type II $\mu_{g g}^{h}(Z Z) / \mu_{g g}^{h}(\gamma \gamma)>1$ always. Likewise, there are complementary correlations between the ggF and VBF modes, as illustrated for the $\gamma \gamma$ final state in the lower row of Fig. 5 . In particular, if $\mu_{\mathrm{VBF}}^{h}(\gamma \gamma)>1$, then $\mu_{g g}^{h}(\gamma \gamma)<1$ is required in Type I, whereas just the opposite statement applies in Type II. In general, the cross correlations between different production $\times$ decay modes carry interesting information because of the dependences in particular of the $h g g$ and $h \gamma \gamma$ couplings on $C_{U}$, $C_{V}$ (and in Type II for large $\tan \beta$ also on $C_{D}$ ) and thus can be useful for distinguishing scenarios. 

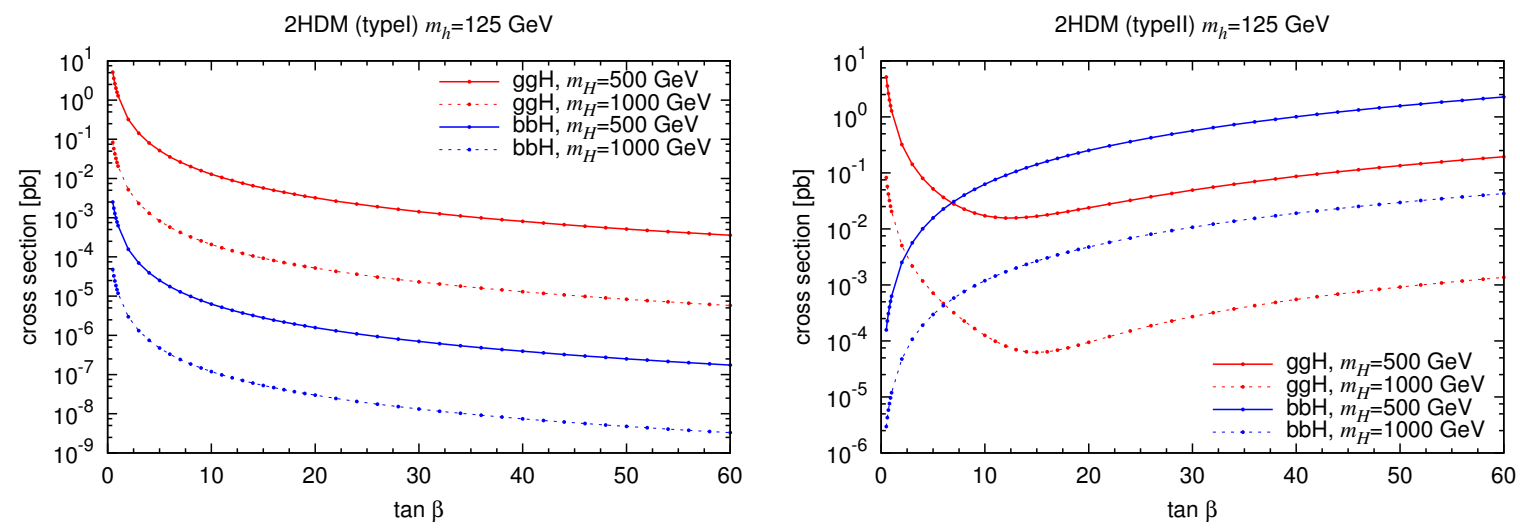

FIG. 6: Cross sections (in pb) for $g g \rightarrow H$ and $b b H$ production at $\sqrt{s}=8 \mathrm{TeV}$ as a function of $\tan \beta$ for $m_{H}=500 \mathrm{GeV}$ and $1 \mathrm{TeV}$. The results shown are for the SM limit of $\sin (\beta-\alpha)=1$.

With regard to the $H / A$ limits coming from heavy Higgs bosons, we find that they have significant impact. In the case of Type I, the limits coming from $g g \rightarrow H, A \rightarrow \tau \tau$ are always stronger (even at high $\tan \beta$ ) than those coming from $g g \rightarrow b \bar{b} H, b \bar{b} A$ with $H, A \rightarrow \tau \tau$. This is because, in Type I models, all the fermionic couplings are the same and it is only a question of the $\tan \beta$-independent ratio of the $g g \rightarrow H, A$ cross section to the $g g \rightarrow b \bar{b} H, b \bar{b} A$ cross section. This ratio is always quite a bit larger than 1 (by typically a factor of at least 100) at any given mass. For Type II models, we note that the down-type coupling is enhanced by $\tan \beta$ and affects both production modes, in the $g g \rightarrow H, A$ case by enhancing the $b$-quark contribution to the one-loop coupling. To illustrate the comparison between these two production modes, we plot for two representative masses (500 GeV and $1 \mathrm{TeV})$ the two cross sections vs. $\tan \beta$ in Fig. 6 with $\alpha$ chosen so that $\sin (\beta-\alpha)=1$. In the case of Type II, we observe that at low $\tan \beta$ it is $\sigma(g g \rightarrow H)$ that is biggest while at high $\tan \beta$ it is $\sigma(b b H)$ that is biggest by a factor of $\sim 10$ to 100 .

\section{B. Implications for the future}

At this point, we turn to a consideration of what future measurements at LHC 13/14 or a linear collider might be most revealing. Let us first quantify the extent to which future higher precision measurements at the next LHC run might be able to restrict the model parameter space. Typical results are illustrated in Fig. 7. To make clear the impact of increased precision in the future, we will show points that survive if the observed values of 

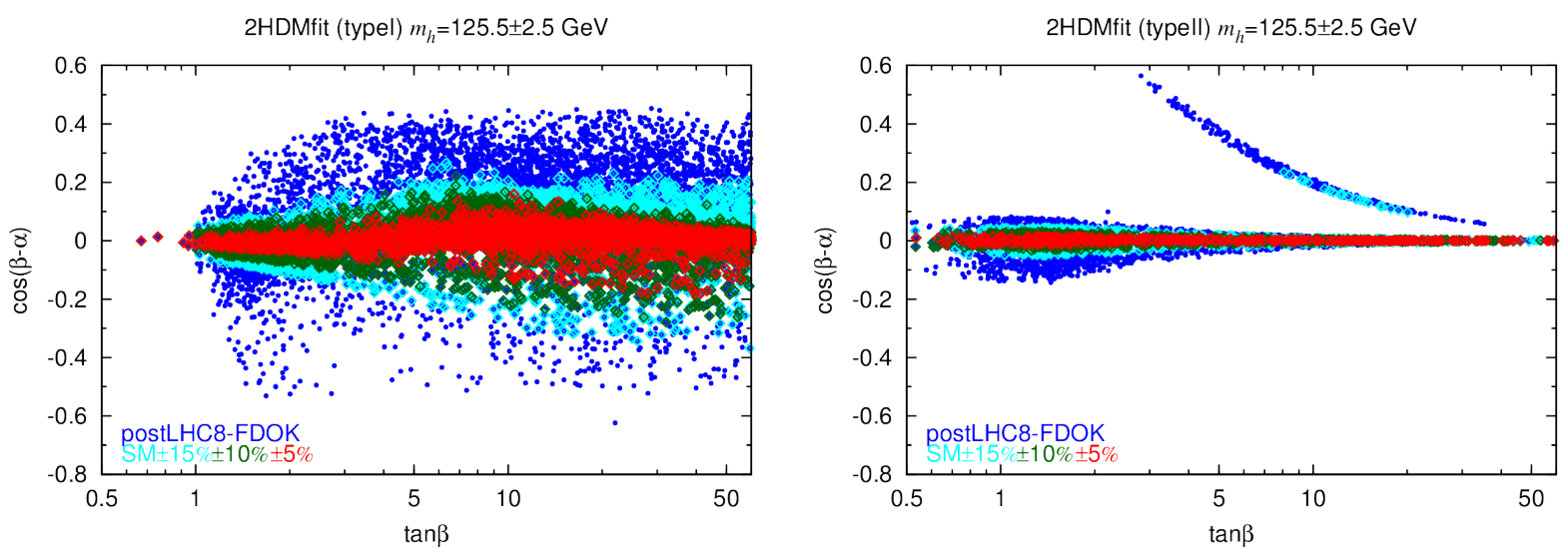

FIG. 7: The postLHC8-FDOK points in the $\cos (\beta-\alpha)$ vs. $\tan \beta$ plane for the $m_{h} \sim 125.5 \mathrm{GeV}$ scenario comparing current $h$ fits (blue) to the case that the rates for all the channels listed in Eq. (6) are within $\pm 15 \%$ (cyan), $\pm 10 \%$ (green) or $\pm 5 \%$ (red) of the SM Higgs prediction. FDOK is also required for these latter points.

$\mu_{X}^{h}(Y)$ all lie within $P \%$ of the SM prediction for the following channels $(X, Y):^{5}$

$$
(g g, \gamma \gamma),(g g, Z Z),(g g, \tau \tau),(\mathrm{VBF}, \gamma \gamma),(\mathrm{VBF}, Z Z),(\mathrm{VBF}, \tau \tau)=(\mathrm{VH}, b b),(\mathrm{ttH}, b b)
$$

Here, we will consider $P= \pm 15 \%, \pm 10 \%$ and $\pm 5 \%$. For this we use the shorthand notation $\mathrm{SM} \pm 15 \%, \mathrm{SM} \pm 10 \%$ and $\mathrm{SM} \pm 5 \%$, respectively. Not unexpectedly, as increasingly precise agreement with the SM is imposed in the various channels, one is quickly pushed to small $|\cos (\beta-\alpha)|$, but $\tan \beta$ remains unrestricted. Note that even $\mathrm{SM} \pm 10 \%$ on each of the individual $\mu$ 's will have eliminated the "wrong-sign" down-quark Yukawa region (which corresponds to $\sin \alpha>0$ or $\left.C_{D}^{h}<0\right)$ of the Type II model.

The reason for this is clarified by Fig. 8. Here, we require that the rates for the $b b$ and $\tau \tau$ final states are consistent at 95\% C.L. with current data and then examine the implications for $g g \rightarrow h \rightarrow \gamma \gamma$ when requiring that the $g g \rightarrow h \rightarrow Z Z$ and $V V \rightarrow h \rightarrow Z Z$ rates be progressively closer to the $\mathrm{SM}$ prediction. We see that once $\mathrm{SM} \pm 10 \%$ is required for the $Z Z$ final state, then $\mu_{g g}^{h}(\gamma \gamma)$ is at least $5 \%$ below the SM in the $\sin \alpha>0$ region. As explained in [41] this is because for $\sin \alpha>0$ the charged-Higgs loop contribution to the $h \gamma \gamma$ coupling

\footnotetext{
${ }^{5}$ It is important to note that the $(\mathrm{VBF}, \tau \tau)=(\mathrm{VH}, b b)$ channels have exactly the same scaling factor in 2HDMs. We mention them together since they are experimentally very different channels and although individually they may not be measurable with a certain level of accuracy, in combination they should be able to determine the common $\mu$ to the specified accuracy.
} 

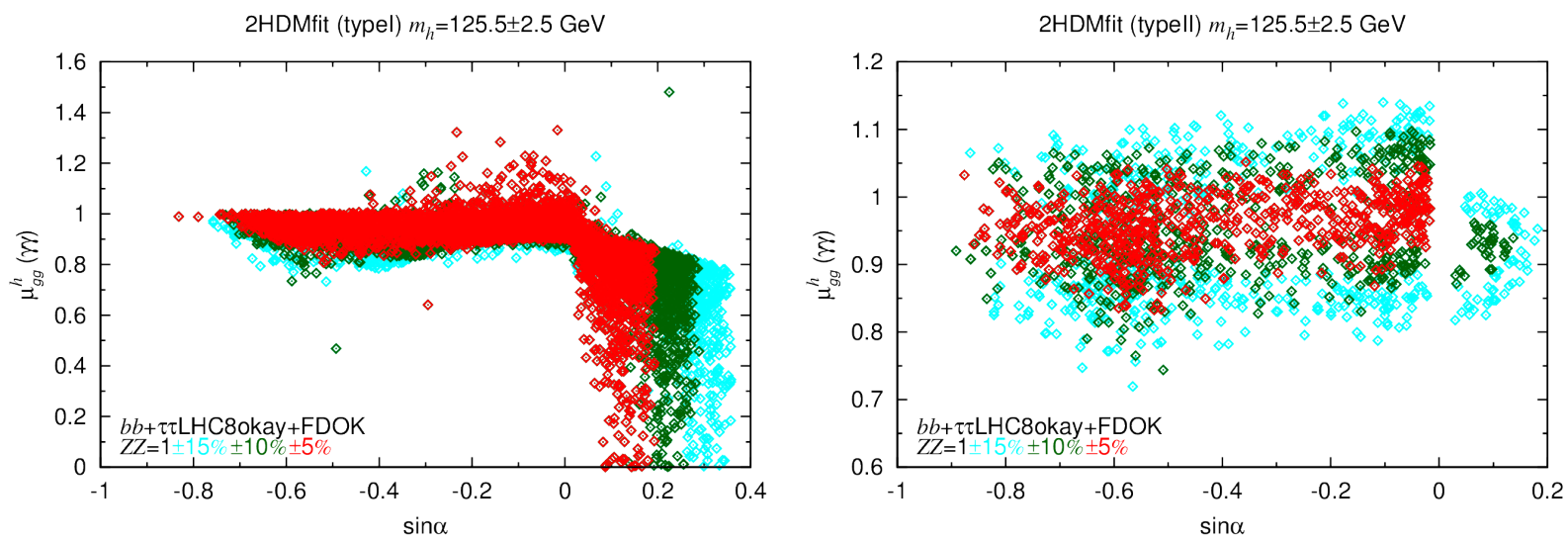

FIG. 8: The postLHC8-FDOK points in the $\mu_{g g}^{h}(\gamma \gamma)$ vs. $\sin \alpha$ plane for the $m_{h} \sim 125.5 \mathrm{GeV}$ scenario, requiring that the $g g \rightarrow Z Z$ and $V V \rightarrow Z Z$ rates are both within $\pm 15 \%$ (cyan), $\pm 10 \%$ (green) and $\pm 5 \%$ (red) of the SM predictions. The individual rates for the $\tau \tau$ and $b b$ final states are consistent with current 95\% CL limits.

does not decouple and causes a decrease in $\Gamma(h \rightarrow \gamma \gamma)$ of order $10 \%$. This decrease is only partially compensated by a $6 \%$ increase in the cross section for $g g \rightarrow h$ that arises due to the fact that the interference between the top and bottom loops has a sign that is opposite the normal sign in this $\sin \alpha>0$ region. As regards the Type I model, the sign of the up-type and down-type Yukawa couplings is independent of the $\operatorname{sign}$ of $\sin \alpha$. Nonetheless, $\sin \alpha>0$ values are disfavored since the $\mu_{g g}^{h}(\gamma \gamma)$ rate reduces as $\sin \alpha$ increases past zero. This is in fact also true in Type II models. As noted in relation to Fig. 1, this is a result of requiring that all quartic Higgs couplings remain perturbative, defined by having absolute values below $4 \pi$.

One quantity of particular interest for a SM-like $h$ is the triple-Higgs coupling strength $\lambda_{h h h}$. We plot the current and possible future expectations in Fig. 9 for $C_{h h h}$ (defined as the value of $\lambda_{h h h}$ relative to the SM value). We observe that if the $\mu_{X}(Y)$ measurements were to have excursions from the SM predictions at the currently allowed $95 \%$ level extreme, then measurement of a large deviation from $C_{h h h}=1$ would be quite likely (also see [42]). For example, at the high-luminosity LHC14, with $L=3000 \mathrm{fb}^{-1}$ one can measure $\lambda_{h h h}$ to the $50 \%$ level [43], and given the limited constraints on the model implied by current Higgs data, deviations from $C_{h h h}=1$ of this order, indeed up to $100 \%$ or more, are possible. However, if future LHC measurements imply increasingly smaller deviations from $\mu_{X}(Y)=1$ in the 

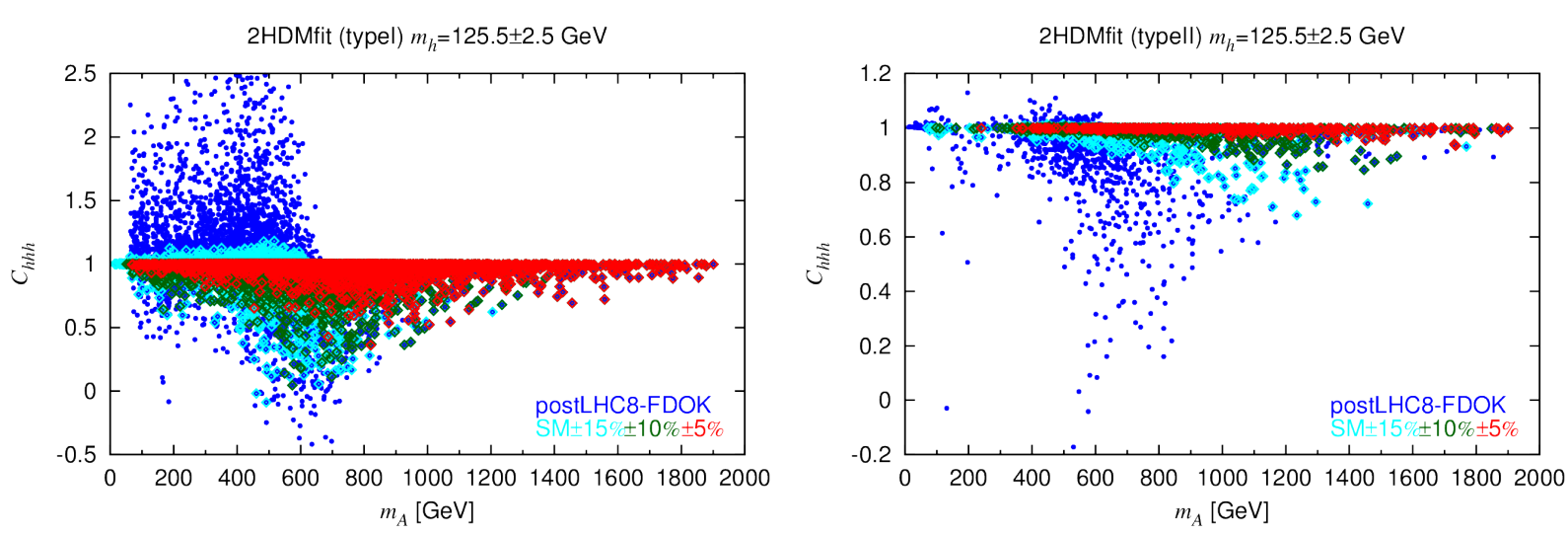

FIG. 9: The postLHC8-FDOK points in the $C_{h h h}$ vs. $m_{A}$ plane for the $m_{h} \sim 125.5 \mathrm{GeV}$ scenario comparing current $h$ fits to the case where future measurements show that the rates for all the channels listed in Eq. (6) are within $\pm 15 \%, \pm 10 \%, \pm 5 \%$ of the SM Higgs prediction; FDOK is required in all cases. Color scheme is as for Fig. 7 .

various channels, then observing a deviation from $C_{h h h}=1$ becomes increasingly difficult, even at the ILC. For example, from [43] we find that the predicted precision on $\lambda_{h h h}$ for ILC1000 with $L=500-1000 \mathrm{fb}^{-1}$ of $21 \%$ and for ILC1000 with $L=1600-2500 \mathrm{fb}^{-1}$ is of order $13 \%$. At CLIC3000 with $L=2000 \mathrm{fb}^{-1}$ the accuracy achievable would be about $10 \%$.

Comparing to the deviations shown in Fig. 9, we see that in Type I a determination of $\mu_{X}(Y)$ rates at the level of $\mathrm{SM} \pm 10 \%$ still allows $C_{h h h}$ as small as $\sim 0$, while $\mathrm{SM} \pm 5 \%$ allows $C_{h h h}$ as small as 0.3, either of which will be observable for any of the listed machines and integrated $L$ values. In contrast, for Type II, even $\mathrm{SM} \pm 15 \%$ would already imply that $C_{h h h}$ must lie below 1. This agrees with the conclusion reached in [17] where it is stated that current $68 \%$ C.L. $(1 \sigma)$ limits (which are very close to our $\mathrm{SM} \pm 15 \%$ constraint) imply $C_{h h h} \leq 1$ for Type II. We note further that the smallest $C_{h h h}$ for $\mathrm{SM} \pm 10 \%$ is $\sim 0.9$, while for $\mathrm{SM} \pm 5 \%$ it is $\sim 0.95$. The former would require CLIC3000 while the latter would be beyond the reach of any of the above $e^{+} e^{-}$colliders. Thus, it is clear that future LHC Higgs data could have a very significant impact on the prospects for seeing an interesting deviation from $C_{h h h}=1$ at ILC/CLIC. As an aside, we note from Fig. 9 that for Type II (but not Type I) models $\mathrm{SM} \pm 5 \%$ is only possible for $m_{A} \gtrsim 250-300 \mathrm{GeV}$ depending on $\tan \beta$.

With this in mind, it is important to consider implications of the current and future $h$ fits for the heavier Higgs bosons. Hopefully, one will retain a significant possibility of detecting 

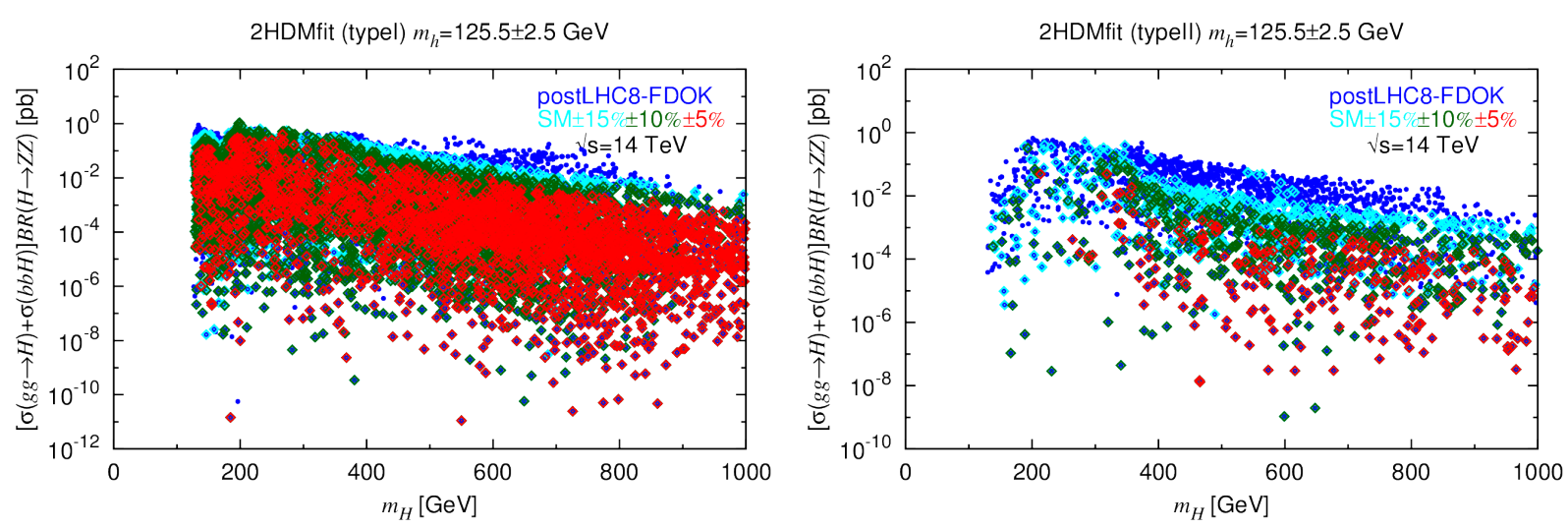

FIG. 10: We plot $[\sigma(g g \rightarrow H)+\sigma(b b H)] \mathrm{BR}(H \rightarrow Z Z)$ as functions of $m_{H}$, for Type I (left) and Type II (right) 2HDMs. Only FDOK points are shown. Implications of various levels of precision for future $h$ measurements are displayed. Color scheme is as for Fig. 7 .

the heavier Higgs bosons even if the $h$ is shown to be very SM-like. To assess the situation, we consider only the high-rate gluon-fusion and $b b$ associated production processes for the $H$ and $A .{ }^{6}$ There are many final states of potential interest. These include $H \rightarrow Z Z$, $H, A \rightarrow \tau \tau, \gamma \gamma, t \bar{t}$ as well as the $H \rightarrow h h$ and $A \rightarrow Z h$ final states.

Results for $H \rightarrow Z Z$ at $\sqrt{s}=14 \mathrm{TeV}$ are shown in Fig. 10. We observe that substantial $\sigma \times \mathrm{BR}$ values (as high as $\sim 1 \mathrm{pb}$ at $m_{H} \sim 150 \mathrm{GeV}$ and $\sim 1 \mathrm{fb}$ at $m_{H} \sim 1 \mathrm{TeV}$ ) are possible, but certainly not guaranteed. In the case of the Type II model, if the $h$ is determined to have SM-like rates within $\pm 10 \%$ or, especially, $\pm 5 \%$ then the maximum possible $\sigma \times \mathrm{BR}$ is substantially reduced and the minimum allowed $m_{H}$ for $\pm 5 \%$ is of order $200 \mathrm{GeV}$.

Results for $g g+b b \rightarrow H, A$ production in the $\tau \tau$ final state are displayed in Fig. 11 assuming $\sqrt{s}=14 \mathrm{TeV}$. Overall, the range of possible cross sections is quite large, with maximum values of order 1 to $10 \mathrm{pb}$ and minimum values below $10^{-10} \mathrm{pb}$ in the case of Type I (although this range is somewhat narrowed on average as the $h$ is required to be more and more SM-like) and minimum values of order $10^{-4}-10^{-5} \mathrm{pb}$ in the case of Type II. It is worth noting that for lower values of $m_{H}$ and $m_{A},[\sigma(g g \rightarrow H)+\sigma(b b H)] \mathrm{BR}(H \rightarrow \tau \tau)$ and $[\sigma(g g \rightarrow A)+\sigma(b b A)] \operatorname{BR}(A \rightarrow \tau \tau)$ are typically quite substantial in the Type II case,

\footnotetext{
${ }^{6}$ For reasons of space, we will mainly present results for the inclusively summed ggF and $b b$ associated production of a given Higgs for $\sqrt{s}=14 \mathrm{TeV}$. Of course, it will be possible and of interest to separate these experimentally.
} 

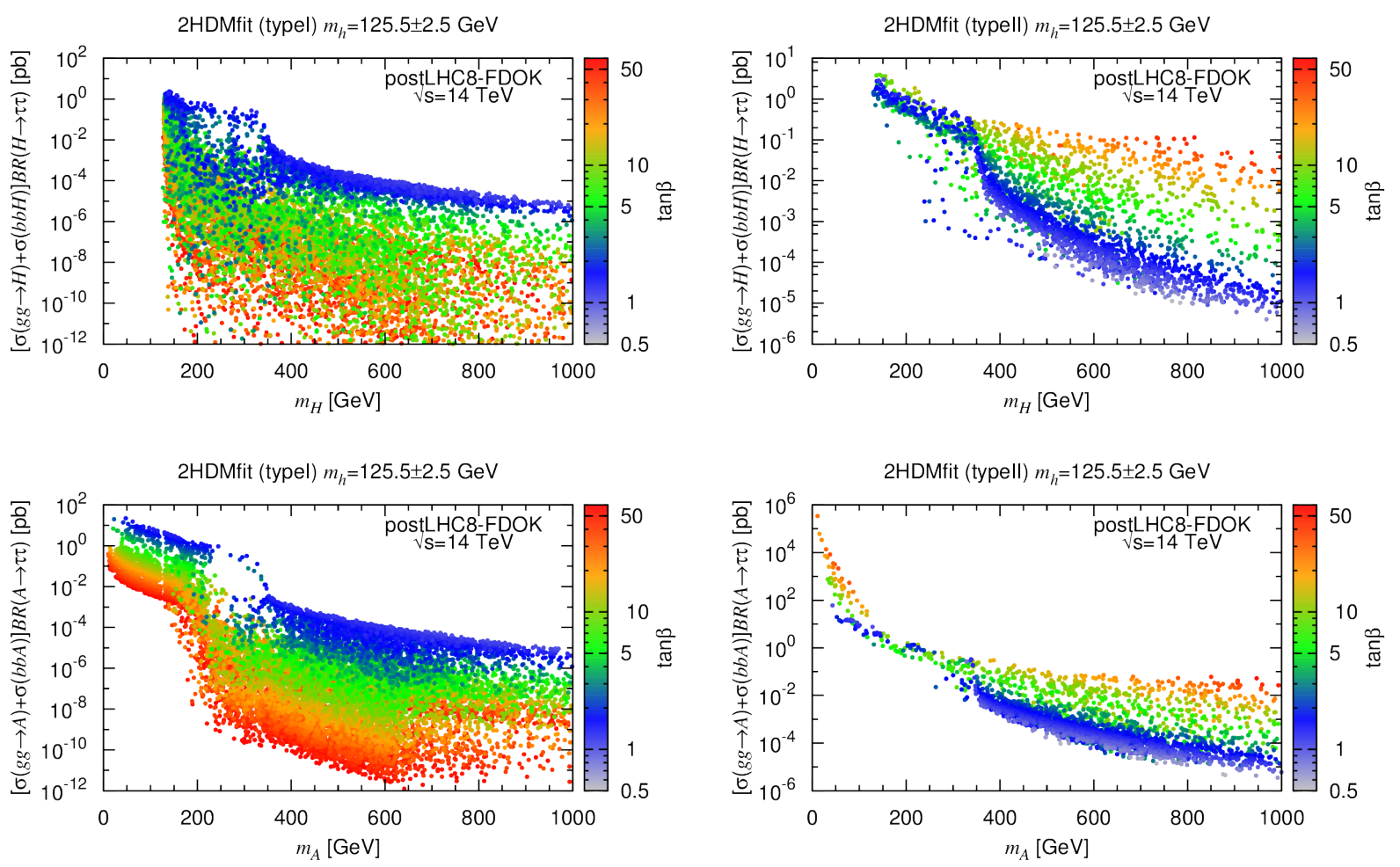

FIG. 11: Scatter plots of $[\sigma(g g \rightarrow H)+\sigma(b b H)] \mathrm{BR}(H \rightarrow \tau \tau)$ and $[\sigma(g g \rightarrow A)+\sigma(b b A)] \mathrm{BR}(A \rightarrow$ $\tau \tau$ ), in pb, as functions of $m_{H}$ (top row) and $m_{A}$ (bottom row), respectively, for postLHC8-FDOK points with $m_{h} \sim 125.5 \mathrm{GeV}$. The values of $\tan \beta$ are color-coded as indicated on the plots.

but that few points survive below $m_{H} \sim 300 \mathrm{GeV}$ if the $125.5 \mathrm{GeV}$ state rates lie within $5 \%$ of the SM Higgs predictions. We comment on one particular feature of the plots, namely the fact that the $t \bar{t}$ threshold is not apparent for Type II in the case of the $A$. This is a direct consequence of the fact that the LHC8 constraints include the limits from [35] on $g g \rightarrow A$ and $b b A$ with $A \rightarrow \tau \tau$. The predicted 2HDM cross sections can significantly exceed these limits in the region below $2 m_{t}$. Since these limits are included in obtaining the postLHC8 results the $t \bar{t}$ threshold that would otherwise be apparent is not present. Note that in the case of the $H$, the limits of [35] do not have a strong impact because the predicted values of $\sigma(g g \rightarrow H) \mathrm{BR}(H \rightarrow \tau \tau)$ are smaller due to the fact that $H \rightarrow Z Z$ decays are also present.

It is also interesting to consider the $\tan \beta$ dependence of the cross sections, indicated by a color code in Fig. 11. As expected from the fermionic couplings in Table I, this dependence is opposite in Type I and Type II. Concretely, in Type I $[\sigma(g g \rightarrow H)+\sigma(b b H)] \mathrm{BR}(H \rightarrow \tau \tau)$ and $[\sigma(g g \rightarrow A)+\sigma(b b A)] \mathrm{BR}(A \rightarrow \tau \tau)$ increase as $\tan \beta$ gets smaller, while in Type II larger 

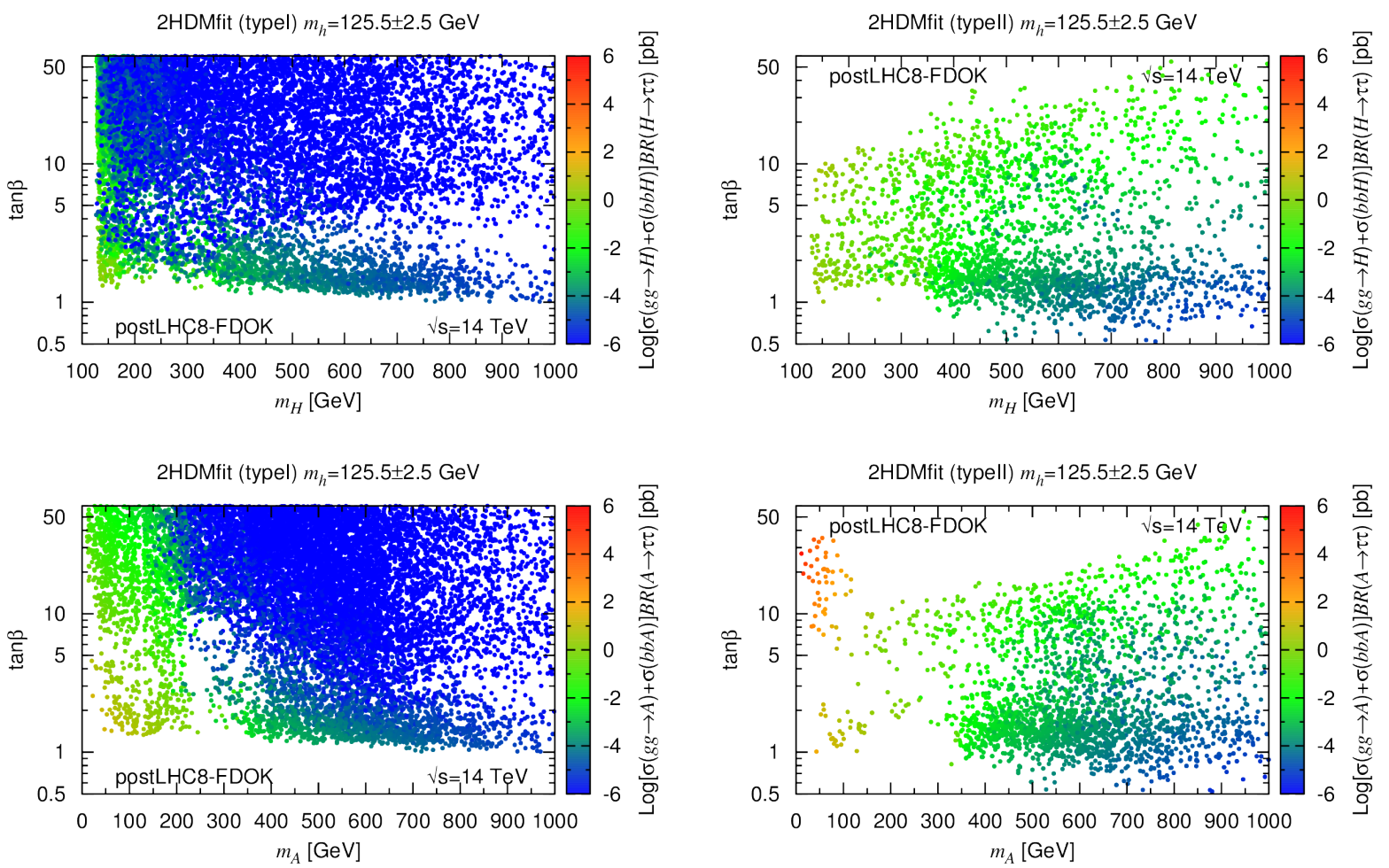

FIG. 12: Same information as in Fig. 11 but in the $\tan \beta$ vs. $m_{H}$ (top row) and $\tan \beta$ vs. $m_{A}$ (bottom row) planes with the $14 \mathrm{TeV}$ cross sections color coded as indicated by the scales on the right of the plots. Only FDOK points are shown.

cross sections are obtained for larger $\tan \beta$. Note also that in Type II the $t \bar{t}$ threshold is visible for small $\tan \beta \lesssim 3$ but not for larger values.

In the case of the Minimal Supersymmetric Standard Model (MSSM), which is a special case of a Type II model, limits on $H, A \rightarrow \tau \tau$ are often presented in the $\tan \beta$ vs. $m_{A}$ plane. For the sake of comparison, we show in Fig. 12 the $H, A \rightarrow \tau \tau$ rates (in pb) in the $\tan \beta$ vs. $m_{H}$ (top row) and $\tan \beta$ vs. $m_{A}$ (bottom row) planes for both Type I and Type II, plotting, however, only points with $\sigma>10^{-6} \mathrm{pb}$. We note a very interesting difference with the MSSM case. In the $2 \mathrm{HDM}$, it is possible to have small $m_{A}$ independent of the other Higgs masses. Further, small $m_{A}$ can escape LEP limits provided, in particular, that $h A$ production is sufficiently suppressed. This is natural in the present case to the extent that $\sin (\beta-\alpha) \sim 1$ for a SM-like $h$ given that the $Z A h$ coupling is $\alpha \cos (\beta-\alpha)$. This is the origin of the points in the bottom right plots of Figs. 11 and 12 with $m_{A} \lesssim 100 \mathrm{GeV}$ and a very large cross section. (Note that CMS and ATLAS $8 \mathrm{TeV}$ constraints on the $\tan \beta$ vs. $m_{A}$ 

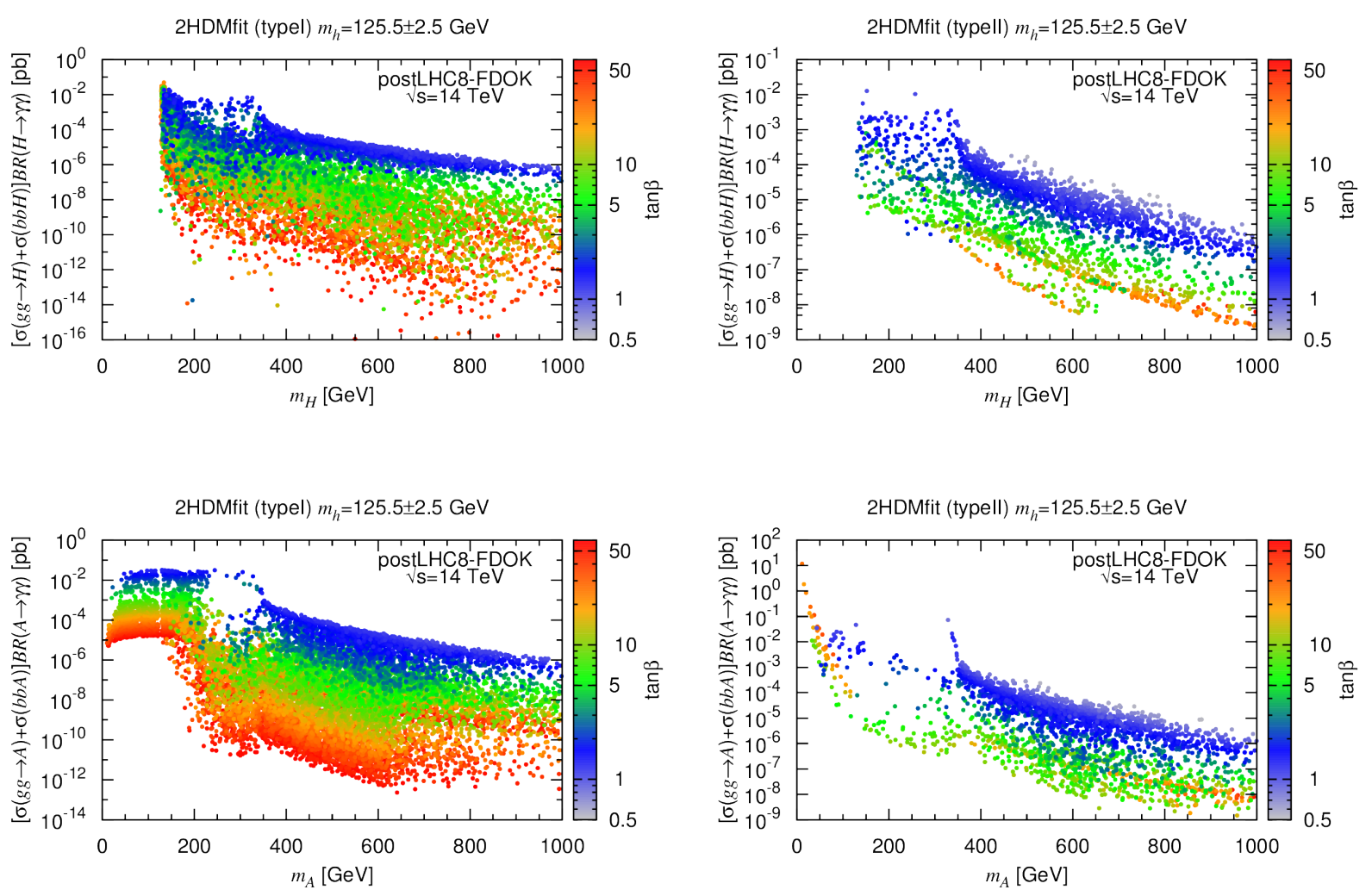

FIG. 13: Scatterplots of $[\sigma(g g \rightarrow H)+\sigma(b b H)] \mathrm{BR}(H \rightarrow \gamma \gamma)$ as function of $m_{H}$ (top row) and $[\sigma(g g \rightarrow A)+\sigma(b b A)] \operatorname{BR}(A \rightarrow \gamma \gamma)$ as function of $m_{A}$ (bottom row), for postLHC8-FDOK points with $m_{h} \sim 125.5 \mathrm{GeV}$. The values of $\tan \beta$ are color coded as indicated by the scales on the right of the plots.

plane (not shown) do not exist below $\sim 90 \mathrm{GeV}$. Furthermore, we have explicitly checked that these limits do allow the few points shown with $m_{A} \gtrsim 90 \mathrm{GeV}$.)

As an aside, we note that in both the $H$ and $A$ cases the $\mu \mu$ final state rates are obtained by simply multiplying by the relevant ratio of branching ratios, $\mathrm{BR}(H$ or $A \rightarrow$ $\mu \mu) / \operatorname{BR}(H$ or $A \rightarrow \tau \tau)$, which is essentially independent of $\tan \beta$ in either Type I or Type II with a value of order $3.5 \times 10^{-3}$. Looking at Fig. 11, it would appear that prospects for detecting the $H$ and $A$ in the $\mu \mu$ final state are significant for $m_{A}$ and $m_{H}$ below the top threshold, especially in the case of $A \rightarrow \mu \mu$ in Type II when $m_{A} \lesssim 150 \mathrm{GeV}$.

Corresponding results for the $H \rightarrow \gamma \gamma$ and $A \rightarrow \gamma \gamma$ final states are shown in Fig. 13 , Aside from $m_{A} \lesssim 50 \mathrm{GeV}$ in Type II, the largest $\sigma \times$ BR's are of order $0.05 \mathrm{pb}$, with much 
lower values being more typical. If the $\gamma \gamma$ continuum background is sufficiently small, $\sigma \times \mathrm{BR}$ values as low as $10^{-3}-10^{-4} \mathrm{pb}$ might well be observable, although it must be kept in mind that the total width of the $H$ or $A$ will be of order several to a few tens of $\mathrm{GeV}$.

In the preceding plots, we have not displayed the impact of future $h$ measurements that lie within $\mathrm{SM} \pm 15 \%, \mathrm{SM} \pm 10 \%$ and $\mathrm{SM} \pm 5 \%$. In the case of Type I, agreement with the SM of $\pm 10 \%$ or better implies that $m_{A} \lesssim 80 \mathrm{GeV}$ is excluded (whereas at the postLHC8 level very low $m_{A}$ is allowed). This is apparent from examining the reach in $m_{A}$ in Fig. 9, In the case of $m_{H}$, which already must lie above $\sim 125.5 \mathrm{GeV}$, there is almost no impact as increasing agreement with the SM is required. For Type II, the impact is more varied. In the case of the $A$, as one moves through $\mathrm{SM} \pm 15 \%, 10 \%, 5 \%$ fewer and fewer points are found at lower $m_{A}$, as can again be read from Fig. 9, but determining precise boundaries would require dedicated scanning. In the case of the $H, \mathrm{SM} \pm 15 \%$ and $\pm 10 \%$ do not restrict $m_{H}$ beyond the postLHC8 range, but heavier $m_{H}$ is preferred by $\mathrm{SM} \pm 5 \%$.

Of course, once $m_{A}$ or $m_{H}$ is above the $t \bar{t}$ threshold, the rates in the $t \bar{t}$ final state will be of great interest. These are shown in Fig. 14. Large $\sigma \times$ BR values are certainly possible, but so also are very small values, although in the case of Type II the smallest values found at $m_{H}$ or $m_{A}$ of order $1 \mathrm{TeV}$ is $\sim 10^{-4} \mathrm{pb}$. This latter might be detectable for full Run2 luminosity of $L=300 \mathrm{fb}^{-1}$, and is certainly of great interest for the high-luminosity run of the LHC which might accumulate $L=3000 \mathrm{fb}^{-1}$.

Perhaps most interesting are the rates for $H \rightarrow h h$ and $A \rightarrow Z h$. First, we show in Fig. 15 the results for ggF at $\sqrt{s}=8 \mathrm{TeV}$. These results should be compared to the $\operatorname{ggF}$ limits obtained recently in [32, which are shown as black lines in the plots of Fig. 15, see also projections at the $14 \mathrm{TeV}$ LHC in [44]. Moreover, we show the points that have significant FD (as discussed in detail in the following subsection). We distinguish between points for which the amount of FD violates the FDOK criteria, but is still moderate in size (orange points, labelled "Low FD"), as defined below in Section IIIC, and points with a high level of FD (red points, labelled "High FD"). In the case of $H \rightarrow h h$, none of the points are excluded by [32], not even the High FD ones. In the case of $\sigma(g g \rightarrow A) \operatorname{BR}(A \rightarrow Z h)$, on the other hand, the experimental limits exclude a significant fraction of the High FD points in the case of Type I models. In the case of Type II, only a few of the High FD points are excluded. Nonetheless, the nearness of the black line limits to the High FD points indicates 

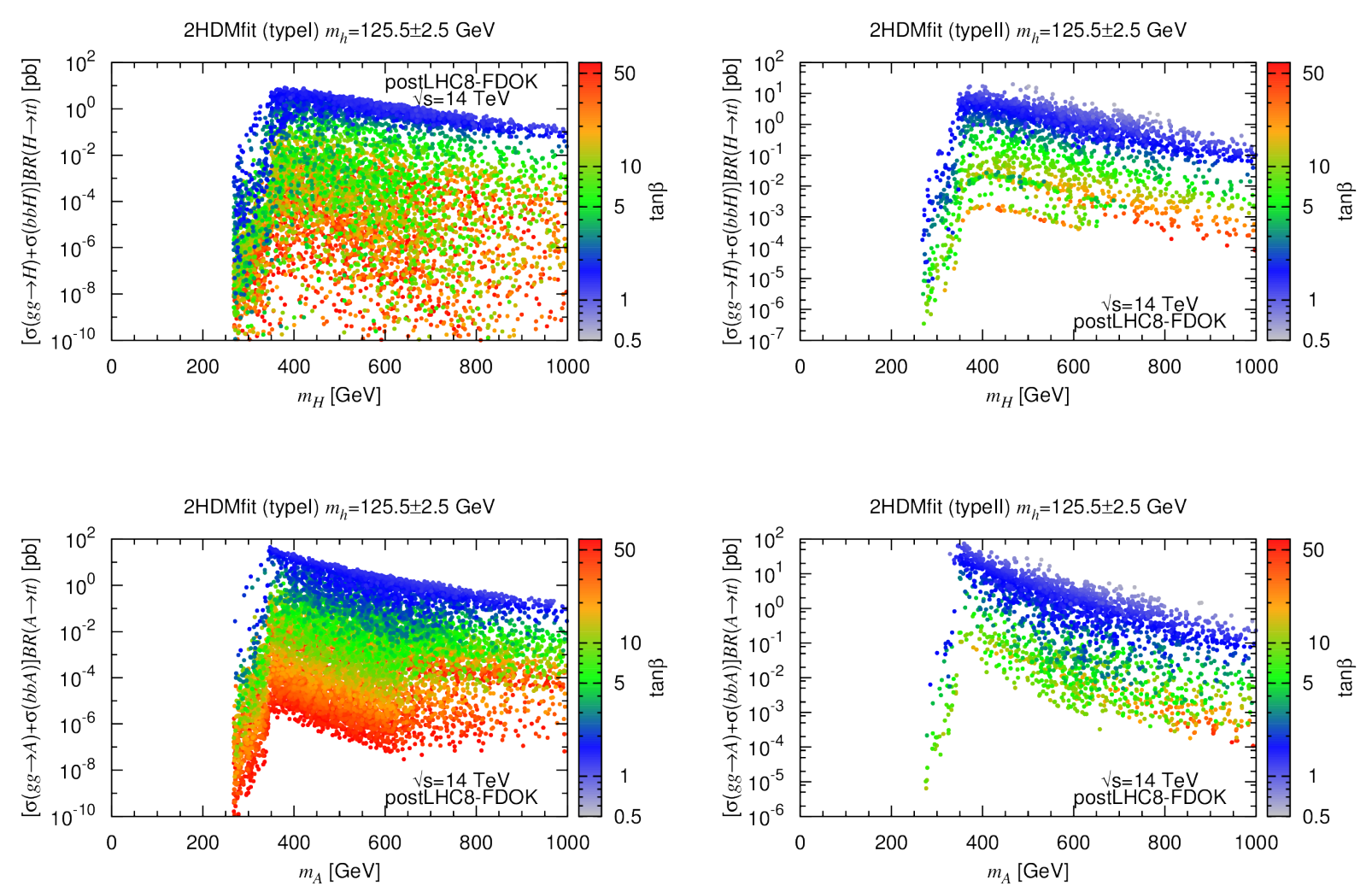

FIG. 14: Scatter plots of $[\sigma(g g \rightarrow H)+\sigma(b b H)] \mathrm{BR}(H \rightarrow t \bar{t})$ as function of $m_{H}$ (top row) and $[\sigma(g g \rightarrow A)+\sigma(b b A)] \mathrm{BR}(A \rightarrow t \bar{t})$ as function of $m_{A}$ (bottom row) at $\sqrt{s}=14 \mathrm{TeV}$, for postLHC8FDOK points with $m_{h} \sim 125.5 \mathrm{GeV}$. The values of $\tan \beta$ are color coded as indicated by the scales on the right of the plots.

that we should not be surprised if the above two processes are the dominant sources of feed down, as we shall describe in the next section.

From Fig. 15 we see that the points with unacceptable FD levels are prominent in the $m_{H} \sim 250-350 \mathrm{GeV}$ region and, especially, in the $m_{A} \sim 200-350 \mathrm{GeV}$ region. Looking back at, for example, Figs. 11 and 13 , which include only postLHC8-FDOK points, we observe corresponding "holes" and depleted regions at low $\tan \beta$ in precisely these mass regions. FD is largest at low $\tan \beta$ where $\sigma(g g \rightarrow A, H)+\sigma(b b A, b b H)$ is largest, as is especially true in the case of Type I models. In these figures, the surviving points that surround or outline the depleted regions are ones with very small $\cos (\beta-\alpha)$ which implies very small $A Z h$ coupling and therefore very small $\mathrm{BR}(A \rightarrow Z h)(A \rightarrow Z h$ being the feed down mechanism of primary 



FIG. 15: We plot $\sigma(g g \rightarrow H) \operatorname{BR}(H \rightarrow h h)$ and $\sigma(g g \rightarrow A) \operatorname{BR}(A \rightarrow Z h)$ as functions of $m_{H}$ and $m_{A}$, respectively, for Type I (left) and Type II (right) 2HDMs. Blue points fulfill all constraints, including the FDOK requirement. Also shown are the points that have Low FD (in orange) and High FD (in red) as defined in Section IIIC. The black lines show the current limits from the CMS analysis of Ref. [32].

importance, see Section III C).

It is interesting to note that to the extent that the $g g \rightarrow A \rightarrow Z h$ process contaminates direct $Z h$ production whereas feed down to the $W h$ final state is substantially smaller (see later discussion), one might observe an apparent violation of custodial symmetry when extracting the $C_{Z}$ and $C_{W}$ effective coupling strengths independently of one another using $Z h$ and $W h$ production, respectively. The fact that the direct limits in Fig. 15 are above the points that have large feed down indicates that apparent custodial symmetry violation might actually be a more sensitive probe of the presence of $H \rightarrow h h$ and, especially, $A \rightarrow Z h$ decays. 

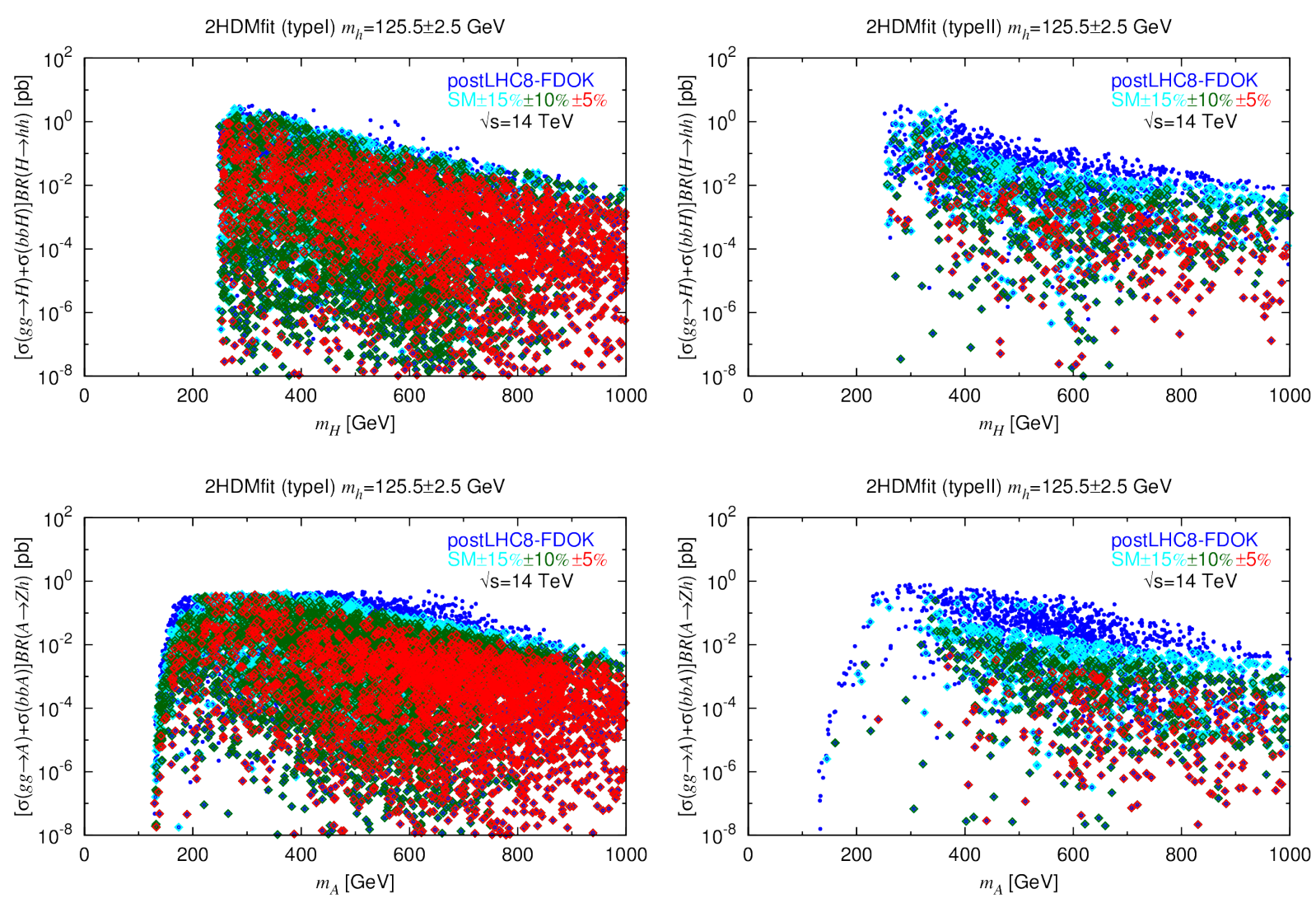

FIG. 16: We plot $[\sigma(g g \rightarrow H)+\sigma(b b H)] \mathrm{BR}(H \rightarrow h h)$ and $[\sigma(g g \rightarrow A)+\sigma(b b A)] \mathrm{BR}(A \rightarrow Z h)$ as functions of $m_{H}$ and $m_{A}$, respectively, for Type I (left) and Type II (right) 2HDMs. For this figure, only FDOK points are shown. Implications of various levels of precision for future $h$ measurements are displayed. Color scheme as in Fig. 7. Values of $\sigma \times \mathrm{BR}$ below $10^{-8}$ are not plotted.

In Fig. 16 we show results for the $h h$ and $Z h$ final states for $\sqrt{s}=14 \mathrm{TeV}$, this time indicating the impact of $\mathrm{SM} \pm 15 \%, \pm 10 \%, \pm 5 \%$ requirements. We note that if the $h$ measurements approach SM values, then this will limit only somewhat the maximum values achievable for the cross section in the $h h$ and $Z h$ final states in the case of Type I models - which means that there is a significant, although not large, probability of seeing the $H \rightarrow h h$ and $A \rightarrow Z h$ final states in gluon fusion and in associated production with $b$ quarks. However, in the case of Type II models, increasingly SM-like $h$ results imply much smaller cross sections than those shown (as allowed by current Higgs fitting for both $h h$ and $Z h$ final states). 


\section{Feed down of heavier Higgs to the $125.5 \mathrm{GeV} h$}

Let us now turn to the issue of whether feed down from heavier Higgs decaying to the $\sim 125.5 \mathrm{GeV}$ Higgs could invalidate our fitting. All the blue points plotted earlier are such that FD is neglectable in all channels of interest. The reason to be concerned is that heavy Higgs bosons have a propensity for decaying to a vector boson plus a Higgs boson or to two Higgs bosons. The most direct cases are $H \rightarrow h h$ and $A \rightarrow Z h$, but there are also chains like $H \rightarrow A A$ followed by $A \rightarrow Z h$ or $H \rightarrow H^{+} H^{-}$with $H^{ \pm} \rightarrow W^{ \pm} h$ and so forth. The full formalism for the feed down calculations is given in Appendix $\mathrm{A}$. For the present purposes, we will consider the most important FD sources and associated ratios

$$
\begin{aligned}
\mu_{\mathrm{ggF} h+\mathrm{bb} h}^{\mathrm{FD}} & \equiv \frac{\sum_{\mathcal{H}=H, A}\left(\sigma_{\mathrm{ggFH}}+\sigma_{b b \mathcal{H}}\right) P_{\mathrm{FD}}(\mathcal{H} \rightarrow h+X)}{\sigma_{\mathrm{ggF} h}+\sigma_{b b h}}, \\
\mu_{Z h}^{\mathrm{FD}} & \equiv \frac{\sigma_{\mathrm{ggF} A} \mathrm{BR}(A \rightarrow Z h)}{\sigma_{Z h}},
\end{aligned}
$$

where $P_{\mathrm{FD}}(\mathcal{H} \rightarrow h+X)$ is the net branching ratio to produce one (or more) $h$ in the $\mathcal{H}=H$ or $A$ decay chains - see Appendix A. Above, $\sigma_{\mathrm{ggFH}}$ and $\sigma_{b b \mathcal{H}}$ refer to the cross sections for $g g \rightarrow \mathcal{H}$ and $b b \mathcal{H}$ associated production respectively, where $\mathcal{H}$ can in the present case be $H$ or $A$.

We emphasize that the amount of FD is computed without accounting for any reduced efficiency for accepting such events into the $125.5 \mathrm{GeV}$ signal as a result of the experimental cuts used to define the $g g \rightarrow h, b b h$ or $Z^{*} \rightarrow Z h$ channels. In practice, it could be that the actual FD after the experimental cuts currently employed to define the various channels is considerably smaller than this maximally conservative estimate.

In Fig. 17, we exhibit a few features of $\mu_{Z h}^{\mathrm{FD}}$ and $\mu_{\mathrm{ggF} h+\mathrm{bb} h}^{\mathrm{FD}}$. The upper two plots show the relative importance of $\mu_{Z h}^{\mathrm{FD}}$ compared to $\mu_{\mathrm{ggF} h+\mathrm{bb} h}^{\mathrm{FD}}$. We observe that the FD to the $Z h$ final state from $A \rightarrow Z h, \mu_{Z h}^{\mathrm{FD}}$, is almost always the largest due to the large $g g \rightarrow A$ production rate compared to the $Z^{*} \rightarrow Z h$ rate that defines the $\mu_{V \mathrm{H}}^{h}(Z h)$ ratio that is the fundamental LHC measurement of interest.

We must now ask what amount of feed down is too large in the ggF $h+\mathrm{bb} h$ and the $Z h$ cases. This, of course, depends upon the accuracy with which the ggF $h+\mathrm{bb} h$ and $Z h$ channels are measured. At LHC8, very roughly, ggF $h+\mathrm{bb} h$ and $Z h$ channels are measured to accuracies of order $15 \%$ and of order $50 \%$, respectively. Thus, we adopt the following criteria: 

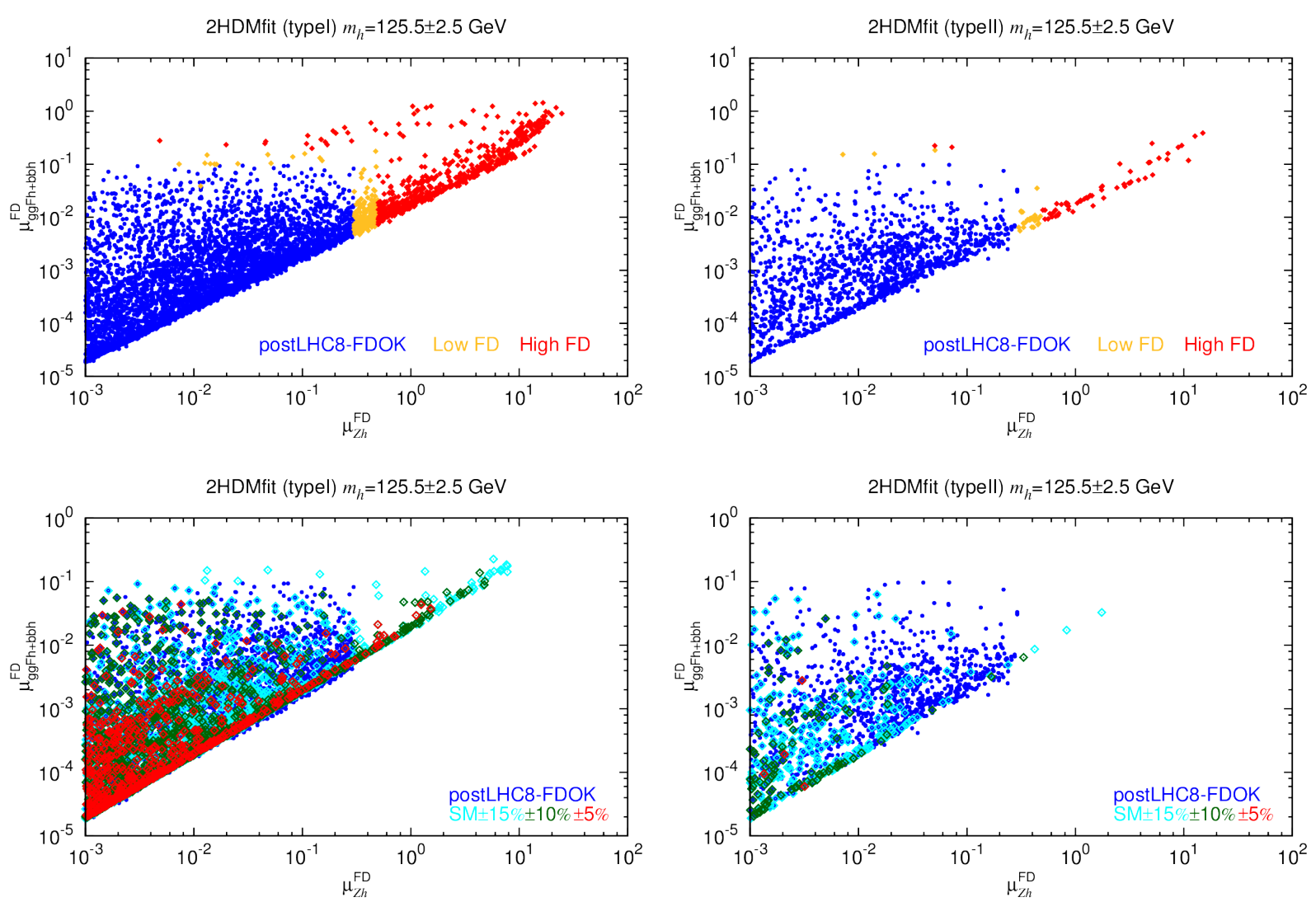

FIG. 17: Scatterplots of $\mu_{\mathrm{ggF} h+\mathrm{bb} h}^{\mathrm{FD}}$ vs. $\mu_{Z h}^{\mathrm{FD}}$. Top plots illustrate how high the FD fractions can go for postLHC8 points (not imposing any FD limit). The plots in the lower row illustrate how convergence of the $h$ properties to SM-like values would limit the maximum possible FDs. We display only points with $\mu_{Z h}^{\mathrm{FD}} \geq 10^{-3}$ - there are many points with much lower values.

- In order for FD not to affect our fits we should have $\mu_{\mathrm{ggF} h+\mathrm{bb} h}^{\mathrm{FD}} \leq 0.1$ and $\mu_{Z h}^{\mathrm{FD}} \leq 0.3$. Points satisfying both criteria were already denoted as "FDOK" above.

- We further define "Low FD" as being cases such that FDOK criteria are violated by virtue of $0.1<\mu_{\mathrm{ggF} h+\mathrm{bb} h}^{\mathrm{FD}} \leq 0.2$ and/or $0.3<\mu_{Z h}^{\mathrm{FD}} \leq 0.5$.

- Finally, "High FD" is defined as $\mu_{\mathrm{ggF} h+\mathrm{bb} h}^{\mathrm{FD}}>0.2$ and $/$ or $\mu_{Z h}^{\mathrm{FD}}>0.5$.

The lower two plots of Fig. 17 illustrate the fact that as the $125.5 \mathrm{GeV}$ resonance is shown to be closer and closer to SM-like in all the various channels the maximum amount of FD that is possible is greatly reduced, becoming quite small for the $\mathrm{SM} \pm 5 \%$ case. Increased precision in the signal strength measurements thus reduces the "danger" of FD contamination. 
To illustrate the effect of FD, we repeat the plots of Figs. 2 and 4 in Fig. 18 showing additional points that have Low FD or High FD in at least one of the channels discussed above. In these plots, the orange (red) points are those for which we have Low FD (High FD). Note that the points in the plots of Fig. 18 with Low FD or High FD all correspond to moderate $\tan \beta$ (up to $\sim 10$ ). However, these points overlap those having an acceptable FD level. Thus, imposing the FD limits does not actually constrain the parameter spaces of the Type I or Type II models. 

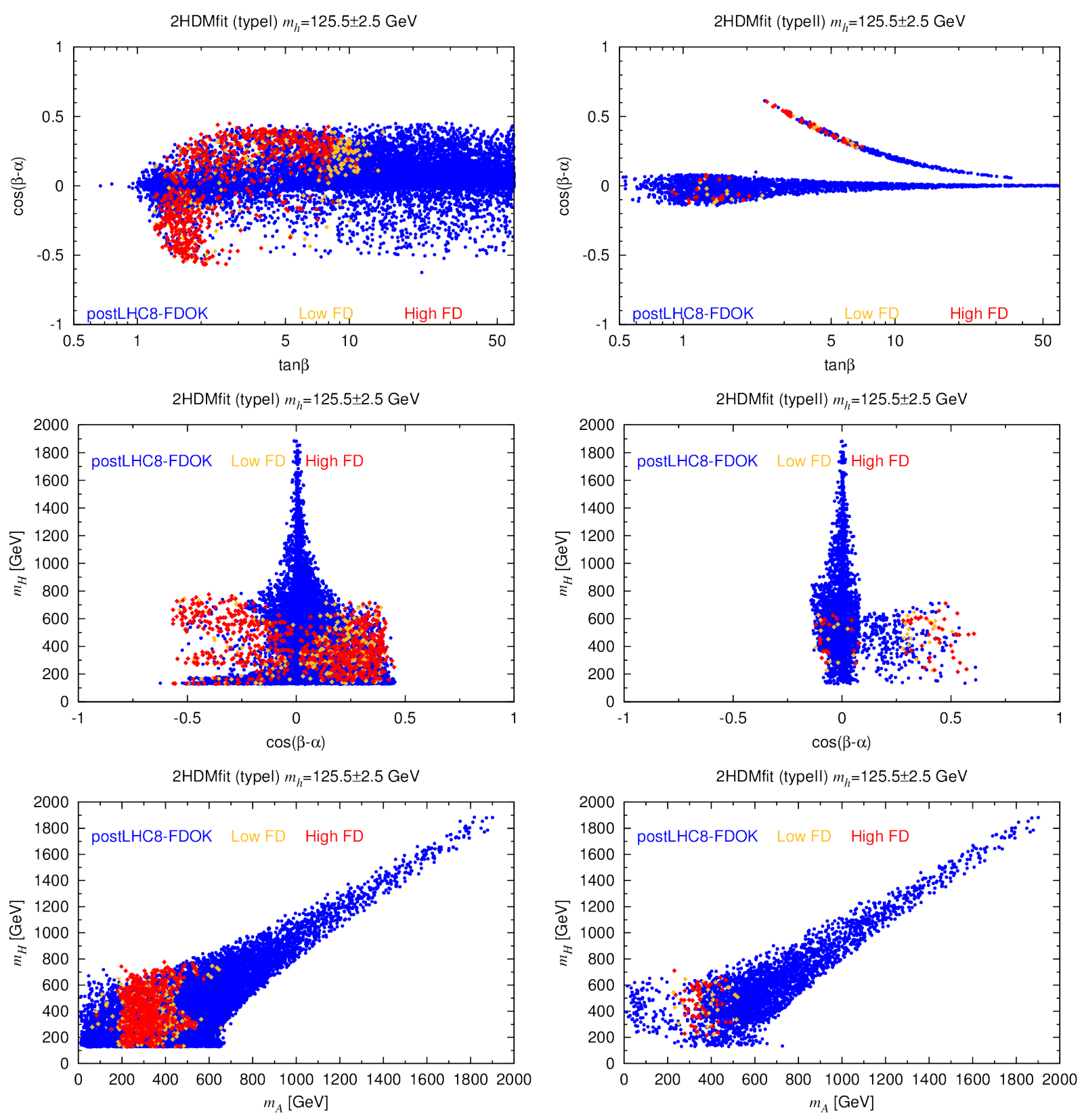

FIG. 18: Constraints in the planes $\cos (\beta-\alpha)$ vs. $\tan \beta$ (top row), $m_{H}$ vs. $\cos (\beta-\alpha)$ (middle row) and $m_{H}$ vs. $m_{A}$ (bottom row) for $m_{h} \sim 125.5 \mathrm{GeV}$. The blue points are the postLHC8-FDOK points. The orange (red) points are allowed postLHC8, but have Low FD (High FD). Many, but not all, Low FD and High FD points have postLHC8-FDOK points hidden below. Regarding the $m_{H}$ vs. $\cos (\beta-\alpha)$ plots, replacing $m_{H}$ by $m_{A}$ or $m_{H^{ \pm}}$gives essentially the same picture. 


\section{IV. $m_{H} \sim 125.5 \mathrm{GeV}$ SCENARIOS}

Let us now turn to the case that the observed SM-like Higgs near $125.5 \mathrm{GeV}$ is the heavier $C P$-even state of the $2 \mathrm{HDM}, H$. In this case, the lighter state, $h$, must have escaped LEP searches. However, we will see that a signal for the $h$ could be hiding in the present data from the LHC and might be revealed in several focused analyses. The pseudoscalar $A$ can be either lighter or heavier than the $H$. Since perturbativity for the quartic couplings prevents the $A$ from being heavier than about $1 \mathrm{TeV}$, it can also give interesting signatures at LHC14.

We begin by presenting in Fig. 19 plots for the $m_{H} \sim 125.5 \mathrm{GeV}$ scenarios analogous to those of Fig. 2 for the $m_{h} \sim 125.5 \mathrm{GeV}$ scenarios. Here, we have again required small FD (from $A$ production and decays); possible FD contributions will be discussed later in this section. In the case of Type I, consistency with the observed $125.5 \mathrm{GeV}$ signal restricts $\sin (\beta-\alpha)$ less than was the case for $\cos (\beta-\alpha)$ in the $m_{h} \sim 125.5 \mathrm{GeV}$ case. In contrast, for Type II the constraints on $\sin (\beta-\alpha)$ are similar in nature to the limits on $\cos (\beta-\alpha)$ in the case of the $h$. There is, however, an important difference. Namely, if $\pm 5 \%$ agreement with the SM can be verified in all the channels listed in Eq. (6), then $m_{H}=125.5 \mathrm{GeV}$ is eliminated in Type II but not in Type I. This can be traced to the fact that the charged-
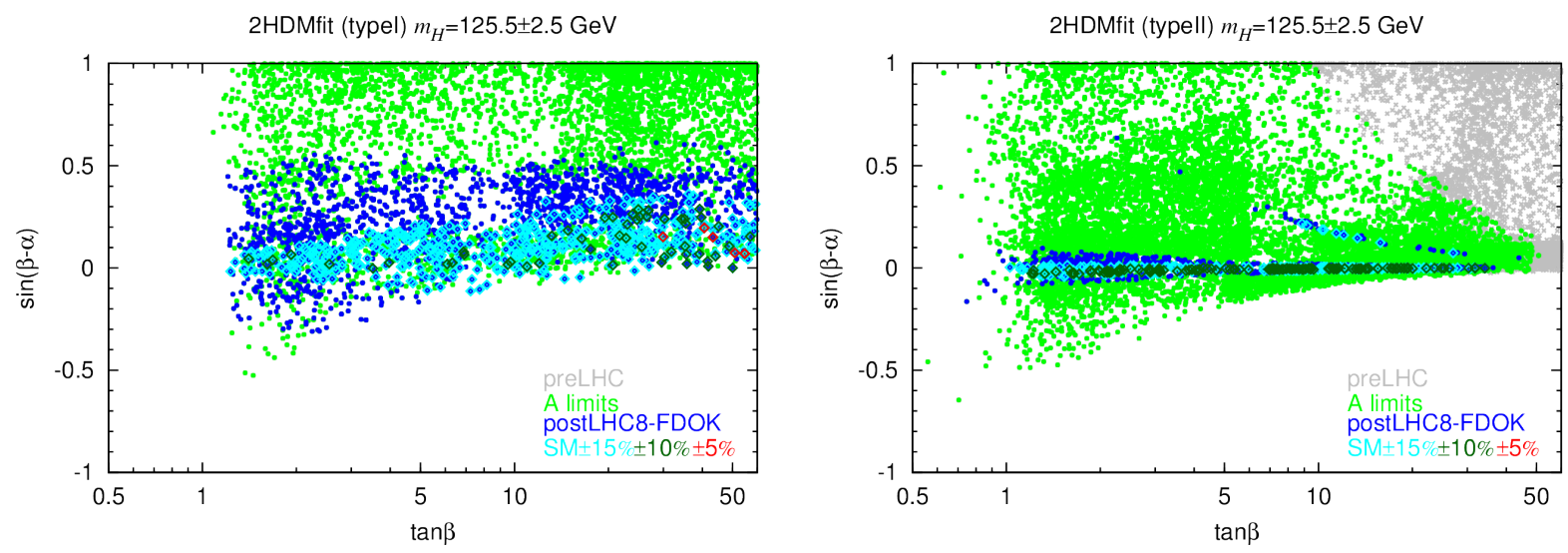

FIG. 19: Constraints on the $2 \mathrm{HDM}$ of Type I and Type II in the $\sin (\beta-\alpha)$ versus $\tan \beta$ plane for $m_{H} \sim 125.5 \mathrm{GeV}$. We show points that survive at the preLHC (grey), A-limits (green), postLHC8FDOK (blue), $\mathrm{SM} \pm 15 \%$ (cyan), $\mathrm{SM} \pm 10 \%$ (dark green), and $\mathrm{SM} \pm 5 \%$ (red) levels. There are no FDOK requirements imposed on the preLHC and A-limits points. The $\mathrm{SM} \pm 15 \%, \pm 10 \%, \pm 5 \%$ points are subjected to FDOK requirements. 

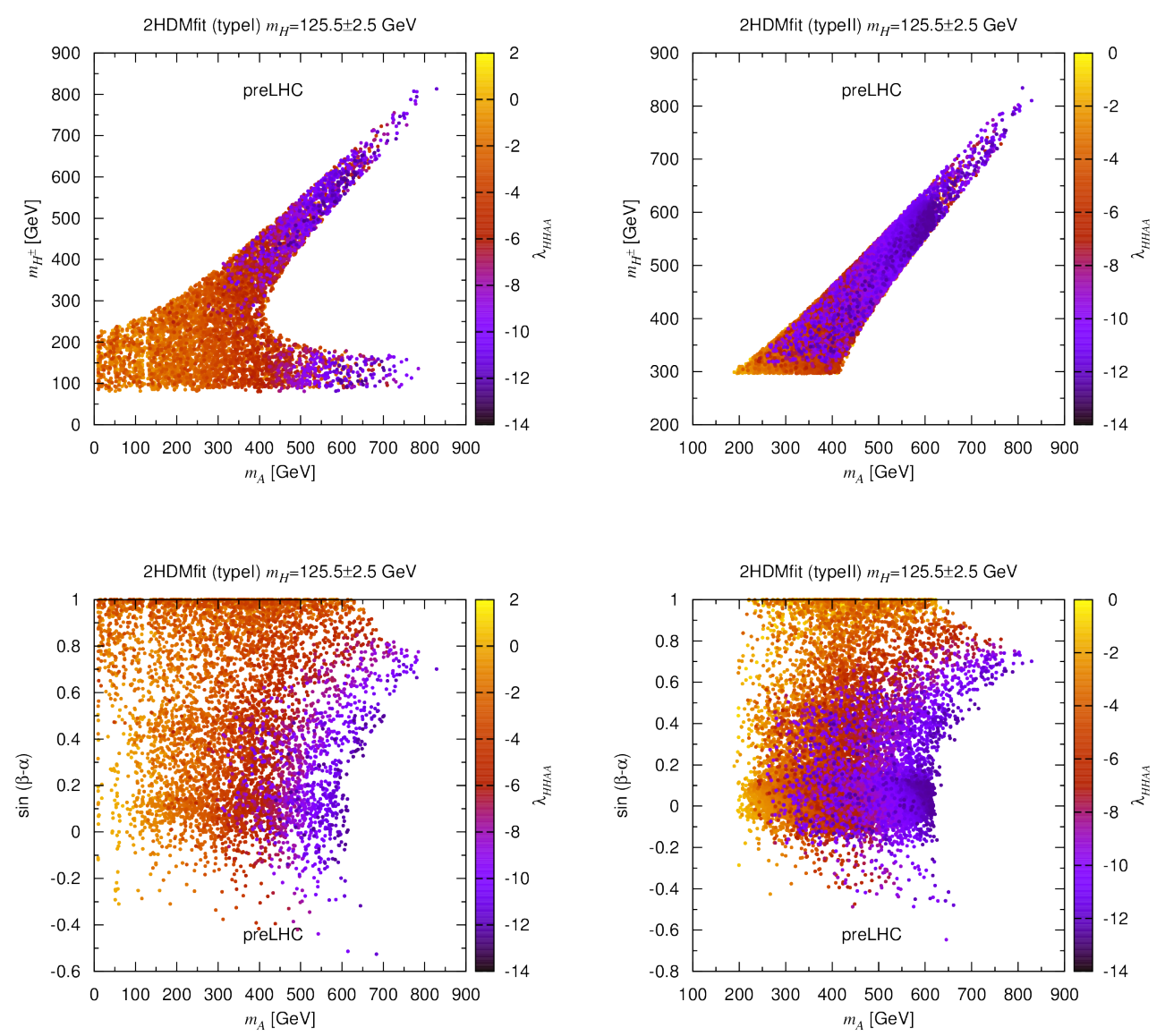

FIG. 20: Scatterplots of $\lambda_{H H A A}$ in the $m_{H^{ \pm}}$vs. $m_{A}$ and $m_{A}$ vs. $\sin (\beta-\alpha)$ planes for the case of $m_{H} \sim 125.5 \mathrm{GeV}$. The values of $\lambda_{H H A A}$ are color coded as indicated by the scales on the right of the plots. The full set of preLHC conditions is satisfied for all points shown.

Higgs loop does not decouple at large $m_{H^{ \pm}}$and ends up suppressing the $H \gamma \gamma$ coupling and therefore the $\gamma \gamma$ final state rates. More details regarding the nondecoupling of the charged Higgs loop contribution to the $H \gamma \gamma$ coupling are presented in Appendix B.

There is a maximum $m_{H^{ \pm}}$that can be achieved before perturbativity is violated, but this maximum applies for all parameter choices, unlike the $m_{h}=125.5 \mathrm{GeV}$ case for which there is a true decoupling limit. To illustrate this, we present in Fig. 20 (upper row) "temperature" plots showing the quartic coupling $\lambda_{H H A A}$ in the plane of $m_{H^{ \pm}}$vs. $m_{A} \cdot \lambda_{H H A A}$ is one of a few that most frequently encounter the perturbativity bound. We see that $\lambda_{H H A A}$ hits its perturbativity bound of $\sim 4 \pi$ at about $m_{A} \sim m_{H^{ \pm}} \sim 800 \mathrm{GeV}$ for both Type I and Type II. In the Type I case the perturbativity limit is also reached at low $m_{H^{ \pm}}$if $m_{A}$ is as heavy as 

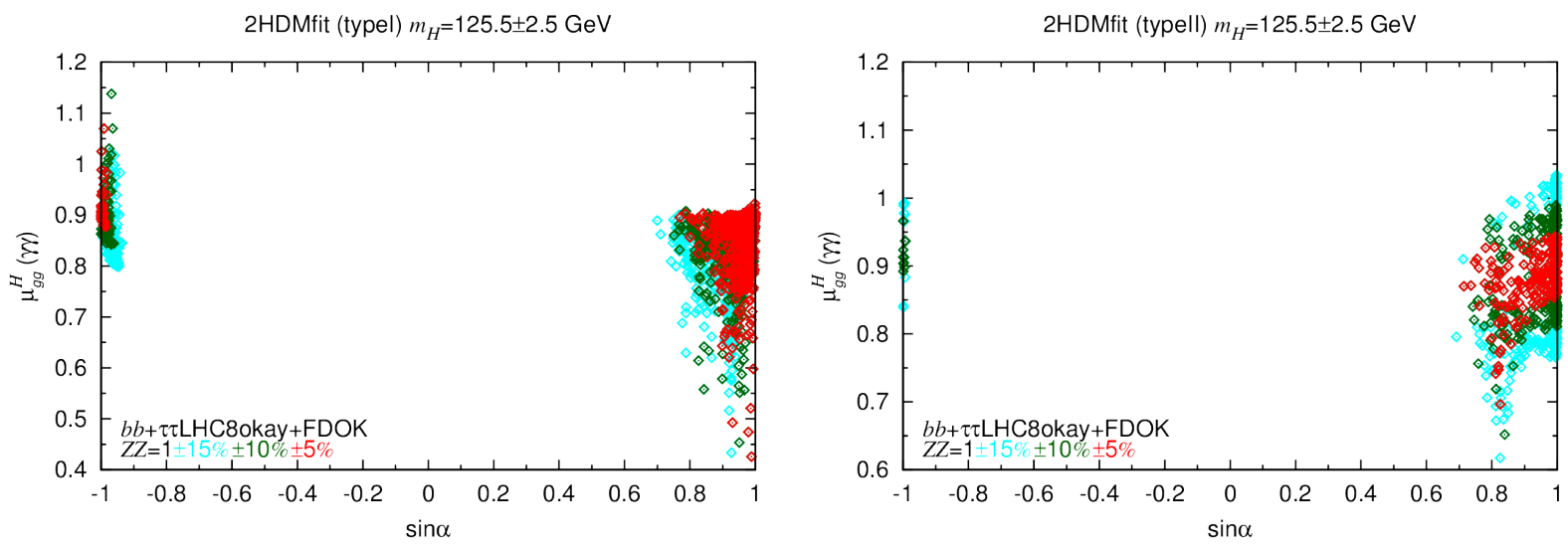

FIG. 21: We plot $\mu_{g g}^{H}(\gamma \gamma)$ as a function of $\sin \alpha$ for points having $g g \rightarrow Z Z$ and $V V \rightarrow Z Z$ rates within $\pm 15 \%, \pm 10 \%$ or $\pm 5 \%$ of the SM predictions.

$\sim 800 \mathrm{GeV}$. This wing of the $m_{H^{ \pm}}$vs. $m_{A}$ plot is not present for Type II because of the lower bound of about $300 \mathrm{GeV}$ from $B$ physics constraints. In the bottom row of Fig. 20 we present temperature plots of $\lambda_{H H A A}$ in the $m_{A}$ vs. $\sin (\beta-\alpha)$ plane, showing that at small to moderate $|\sin (\beta-\alpha)|$ the perturbative bound is already exceeded by $m_{A} \sim 600 \mathrm{GeV}$. In any case, the bottom line is that there is no decoupling limit for the $m_{H}=125.5 \mathrm{GeV}$ case and nondecoupling effects are inevitably of importance.

The resulting $\gamma \gamma$ final state rates are illustrated in Fig. 21. There, we see that consistency with $\pm 5 \%$ for the $Z Z$ final state rates and simultaneously for the $g g \rightarrow H \rightarrow \gamma \gamma$ rate is only possible on the $\sin \alpha<0\left(\right.$ i.e. $\left.C_{U}^{H}<0\right)$ branch in the Type I model. Most of this mismatch can, as said earlier, be traced to the nondecoupling of the charged-Higgs loop contribution to the $H \gamma \gamma$ coupling. In the end only the few red points on the $\sin \alpha<0$ branch of the Type I model having $\mu_{g g}^{H}(\gamma \gamma) \gtrsim 0.95$ can survive if $\leq \pm 5 \%$ deviations from the SM are required for both the $Z Z$ and $\gamma \gamma$ final states.

As regards the $h$ and $A$ masses associated with a good fit by the $H$ to the LHC data and other limits we refer to Fig. 22, There, we see that a proper fit at the postLHC8 level is easily achieved if $m_{h} \gtrsim 60 \mathrm{GeV}$, for which $H \rightarrow h h$ decays are kinematically forbidden. However, there is also a scattering of points for which small values of $m_{h}$ are possible. Such points correspond to parameters for which the Hhh coupling is small. A very "fine-tuned" scan is necessary to find these low- $m_{h}$ points for which $\mathrm{BR}(H \rightarrow h h)$ is small enough that the $H$ signals fit the LHC data at an adequate level. 

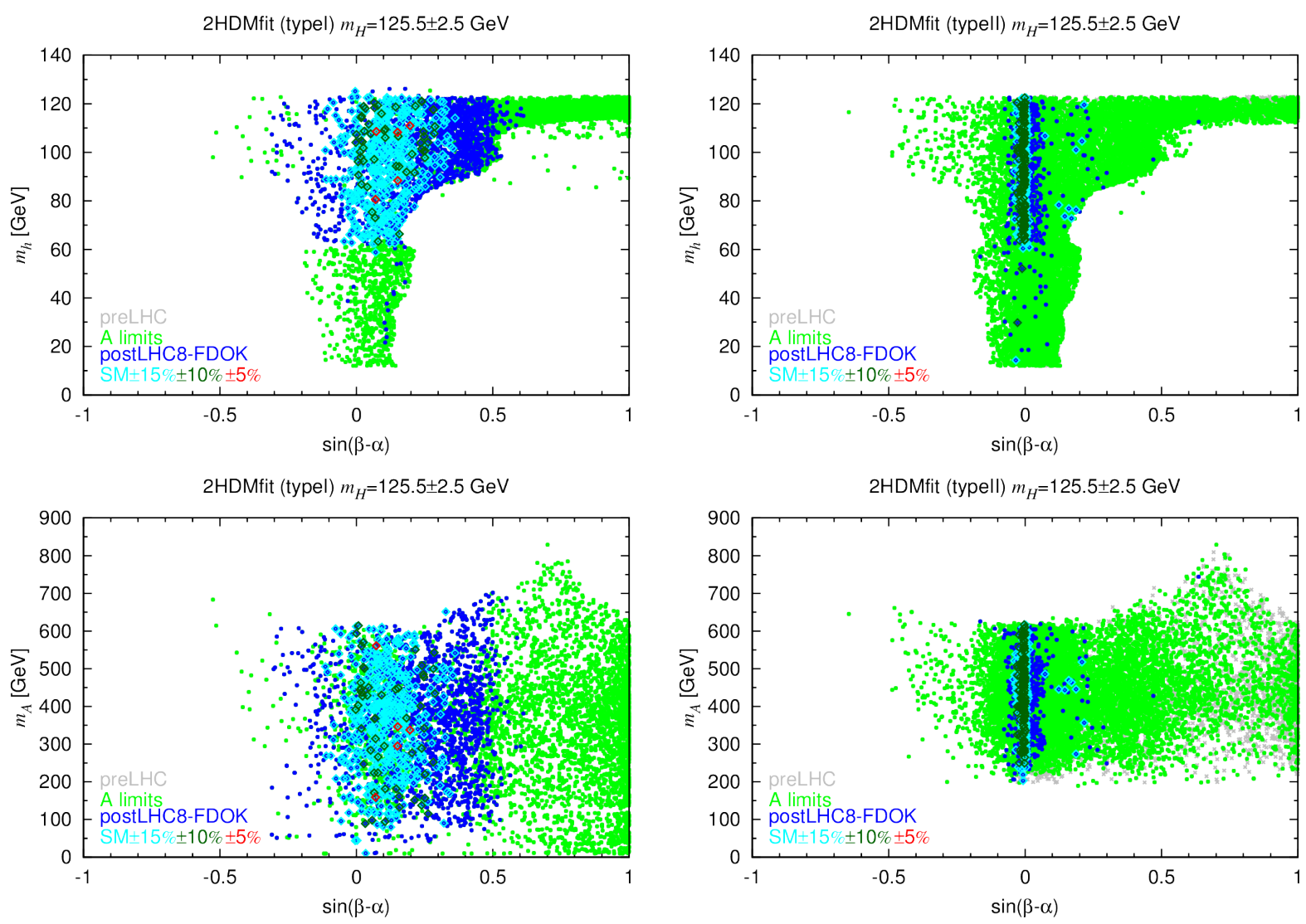

FIG. 22: Constraints in the $m_{h}$ vs. $\sin (\beta-\alpha)$ and $m_{A}$ vs. $\sin (\beta-\alpha)$ planes for the $m_{H} \sim 125.5 \mathrm{GeV}$ scenarios. Results for $m_{H^{ \pm}}$vs. $\sin (\beta-\alpha)$ are very similar to those for $m_{A}$ vs. $\sin (\beta-\alpha)$. There are no FDOK requirements imposed on the preLHC and A-limits points. The $\mathrm{SM} \pm 15 \%, \pm 10 \%, \pm 5 \%$ points are subjected to FDOK requirements.

Let us now address the issue of feed down. Given that $m_{A}$ can be quite large, there is certainly the possibility of $A \rightarrow Z H$ feed down contributions to the $H$ signals. The $H A Z$ coupling is proportional to $\sin (\beta-\alpha)$, which the fits require to be $\lesssim 0.5$ in magnitude. What is important, however, is $\operatorname{BR}(A \rightarrow Z H)=\Gamma(A \rightarrow Z H) / \Gamma_{\text {tot }}(A)$, which can still be large. Figure 23 shows the FD $\mu$ values analogous to those considered for the $m_{h} \sim 125.5 \mathrm{GeV}$ scenario. To be precise, we consider

$$
\begin{aligned}
\mu_{\mathrm{ggF} H+\mathrm{bb} H}^{\mathrm{FD}} & \equiv \frac{\left(\sigma_{\mathrm{ggF} A}+\sigma_{\mathrm{bb} A}\right) P_{\mathrm{FD}}(A \rightarrow H+X)}{\sigma_{\mathrm{ggF} H}+\sigma_{\mathrm{bb} H}}, \\
\mu_{Z H}^{\mathrm{FD}} & \equiv \frac{\sigma_{\mathrm{ggF} A} \mathrm{BR}(A \rightarrow Z H)}{\sigma_{Z H}} .
\end{aligned}
$$

We observe that substantial FD is indeed possible. The ratio defining $\mu_{Z H}^{\mathrm{FD}}$ above, has 

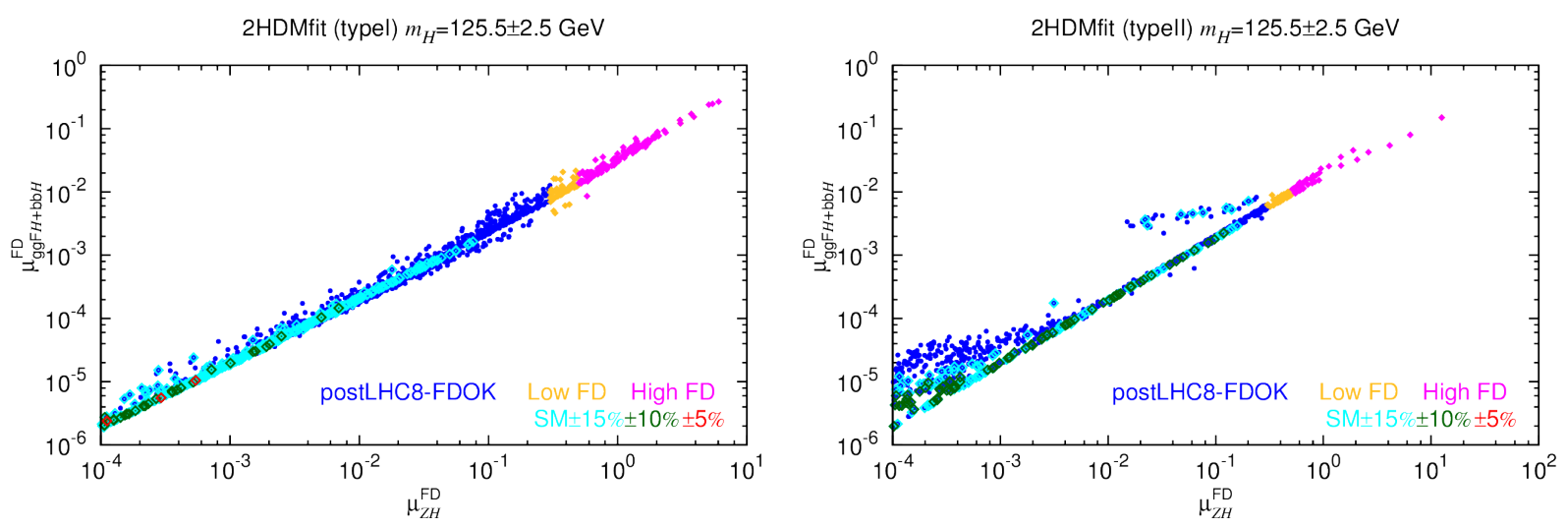

FIG. 23: We plot $\mu_{\mathrm{ggF} H+\mathrm{bb} H}^{\mathrm{FD}}$ vs. $\mu_{Z H}^{\mathrm{FD}}$ illustrating how high FD fractions can go for postLHC8 points for the $m_{H} \sim 125.5 \mathrm{GeV}$ scenarios. Also shown is how convergence of the $H$ properties to SM-like values would limit the maximum possible feed downs. We display only points with $\mu_{Z H}^{\mathrm{FD}} \geq 10^{-4}$ - there are many points with much lower values.

the greatest potential for being large because of the large $g g \rightarrow A$ production rate in the numerator compared to the $Z^{*} \rightarrow Z H$ rate appearing in the denominator. In contrast, in $\mu_{\mathrm{ggF} H+\mathrm{bb} H}^{\mathrm{FD}}$ both numerator and denominator are ggF-dominated. As in the $m_{h}=125.5 \mathrm{GeV}$ case, we exclude from subsequent plots those points which have FD levels that exceed $10 \%$ relative to the $g g \rightarrow H+b b H$ production modes and $30 \%$ in the $Z H$ associated production mode.

As in the case of $m_{h} \sim 125.5 \mathrm{GeV}$, it is interesting to assess the prospects for detecting a deviation in the triple-Higgs coupling as one goes from the current data set to $H$ rates that are increasingly SM-like. In Fig. 24, we plot $\operatorname{sgn}\left(C_{V}^{H}\right) C_{H H H}$, i.e. the ratio of the triple-Higgs coupling $\lambda_{H H_{H}}$ to the value it should have in the SM limit, as a function of $m_{A}$. [We include $\operatorname{sgn}\left(C_{V}^{H}\right)$ because some of the points have $C_{V}^{H}<0$ for our scanning procedure.] We observe that as the LHC signals become increasingly SM-like, the deviations of $\operatorname{sgn}\left(C_{V}^{H}\right) C_{H H H}$ from unity are even more tightly limited than in the case of $C_{h h h}$ for $m_{h} \sim 125.5 \mathrm{GeV}$. Of course, we also see (again) that very few (no) points survive the $\mathrm{SM} \pm 5 \%$ constraint in the case of Type I (Type II).

Let us next assess the feasibility for detecting the lighter $h$. As already noted, finding points with $m_{h} \lesssim 60 \mathrm{GeV}$ for which $\mathrm{BR}(H \rightarrow h h)$ is small enough to still allow the $H$ rates in the various channels to fit the $125.5 \mathrm{GeV}$ signal is highly nontrivial and this scenario 

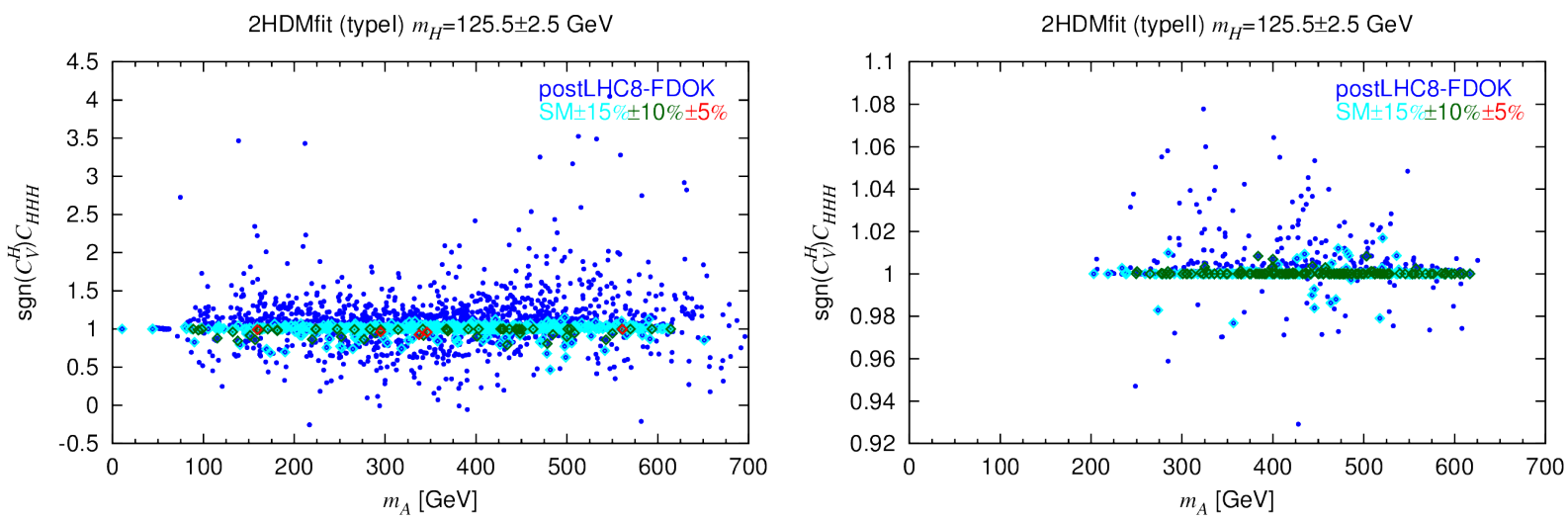

FIG. 24: We display points in the $\operatorname{sgn}\left(C_{V}^{H}\right) C_{H H H}$ vs. $m_{A}$ plane for the $m_{H} \sim 125.5 \mathrm{GeV}$ scenario comparing current $H$ fits to the case where future measurements show that all channel rates are within $\pm 15 \%, \pm 10 \%, \pm 5 \%$ of the SM Higgs prediction; FDOK is required in all cases. Color scheme is as for Fig. 19, except that preLHC and A-limits points are not displayed.

will be discussed in detail elsewhere. The most interesting modes for $h$ detection may be $g g \rightarrow h \rightarrow \gamma \gamma$ and $V^{*} h$ with $h \rightarrow b \bar{b}$. In the context of the current $8 \mathrm{TeV}$ data, only the latter mode is of interest - expected signal strengths as a function of $m_{h}$ are plotted in Fig. 25. While for many points the expected rates are obviously too small to have allowed detection of the $h$, there also exist postLHC8-FDOK points for which detection in the $V h(b \bar{b})$ final state might be on the edge. We speculate that for the $V h(b \bar{b})$ final state, a leptonic trigger on the $V$ might still allow the predicted signal to emerge for the higher $\mu_{\mathrm{VH}}^{h}(b \bar{b})$ values.

As an aside, it is easily inferred from Fig. 25 that the postLHC8 and, even more so, the $\mathrm{SM} \pm 15 \%, \pm 10 \%, \pm 5 \%$ requirements eliminate a large swath of the points that survive the $A$-limits constraint. It is also noteworthy that in the case of Type I all preLHC points automatically satisfy the $A$-limits requirement, whereas some preLHC (grey) points get excluded by the A limits in the case of Type II.

Considering Fig. 25, it is moreover interesting to ask whether the $\gtrsim 2 \sigma$ LEP excess in the $Z b \bar{b}$ final state at $M_{b \bar{b}} \sim 98 \mathrm{GeV}$ could be explained by $m_{h} \sim 98 \mathrm{GeV}$ and $\mu_{\mathrm{VH}}^{h}(b \bar{b}) \sim 0.1-0.3$. We see that this is indeed possible in both the Type I and Type II models given current postLHC8 constraints on the $H$ properties. Of course, the scatterplots suggest that this explanation is more fine-tuned in the Type II case. Furthermore, if the $H$ rates are found to be within $\pm 15 \%$ of the SM rates, the value of $\mu_{\mathrm{VH}}^{h}(b \bar{b})$ is pushed well below the desired 

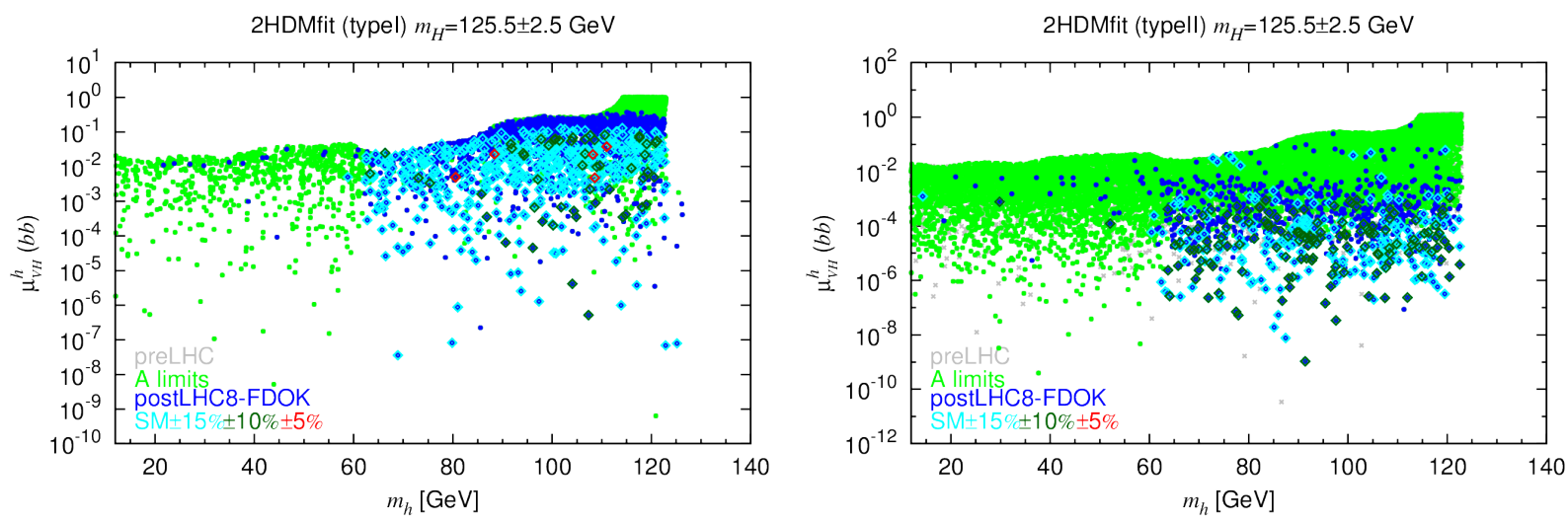

FIG. 25: $\mu_{\mathrm{VH}}^{h}(b \bar{b})$, i.e. $V^{*} \rightarrow V h$ associated production with $h \rightarrow b \bar{b}$ relative to the SM, as a function of $m_{h}$. Note that $\mu_{\mathrm{VH}}^{h}(b b)$ is actually independent of energy and that the ratio also applies to any situation where the subprocess of interest is $V^{*} \rightarrow V h$, including the LEP $Z^{*} \rightarrow Z h$ process. There are no FDOK requirements imposed on the preLHC and A-limits points. The $\mathrm{SM} \pm 15 \%, \pm 10 \%, \pm 5 \%$ points are subjected to FDOK requirements.

range in the case of Type II and is at a marginal level in the case of Type I. At the SM $\pm 5 \%$ level, the few surviving Type I points have $\mu_{\mathrm{VH}}^{h}(b \bar{b}) \lesssim 0.05$ (assuming that a more extensive scan would reveal red points with $m_{h} \sim 98 \mathrm{GeV}$ that would have a signal level comparable to those around $90 \mathrm{GeV}$ and $108 \mathrm{GeV}$ plotted), a value that is not very consistent with the LEP $\sim 2.3 \sigma$ excess observed.

At $14 \mathrm{TeV}$, there is also potential for detecting the $h$ in the $g g \rightarrow h \rightarrow \gamma \gamma$ mode, as shown in Fig. 26. Of course, while a significant event yield is possible for $L \geq 300 \mathrm{fb}^{-1}$, the level of continuum irreducible and reducible backgrounds must be assessed and could prove too large for the blip at $m_{h}$ to be observable.

Finally, let us turn to the question of detecting the pseudoscalar $A$. Figure 27 shows cross sections for pseudoscalar $A$ production, concretely $[\sigma(g g \rightarrow A)+\sigma(b b A))] \times \mathrm{BR}(A \rightarrow \gamma \gamma)$ (top), $\times \mathrm{BR}(A \rightarrow \tau \tau)$ (middle) and $\times \mathrm{BR}(A \rightarrow t \bar{t})$ (bottom) at $14 \mathrm{TeV}$ as a function of $m_{A}{ }^{7}$ Again, there is a large range of possible cross section values at any given $m_{A}$, with the $\tan \beta$ dependence, of course, being the same as for the $m_{h} \sim 125.5 \mathrm{GeV}$ case. As already observed in Fig. 20, the possible range of $m_{A}$ is limited when $m_{H} \sim 125.5 \mathrm{GeV}$.

\footnotetext{
${ }^{7}$ As commented in the last section, we plot the sum as this defines the inclusive production rate. Of course, separating $g g \rightarrow A$ and $b b A$ production processes would eventually be possible.
} 

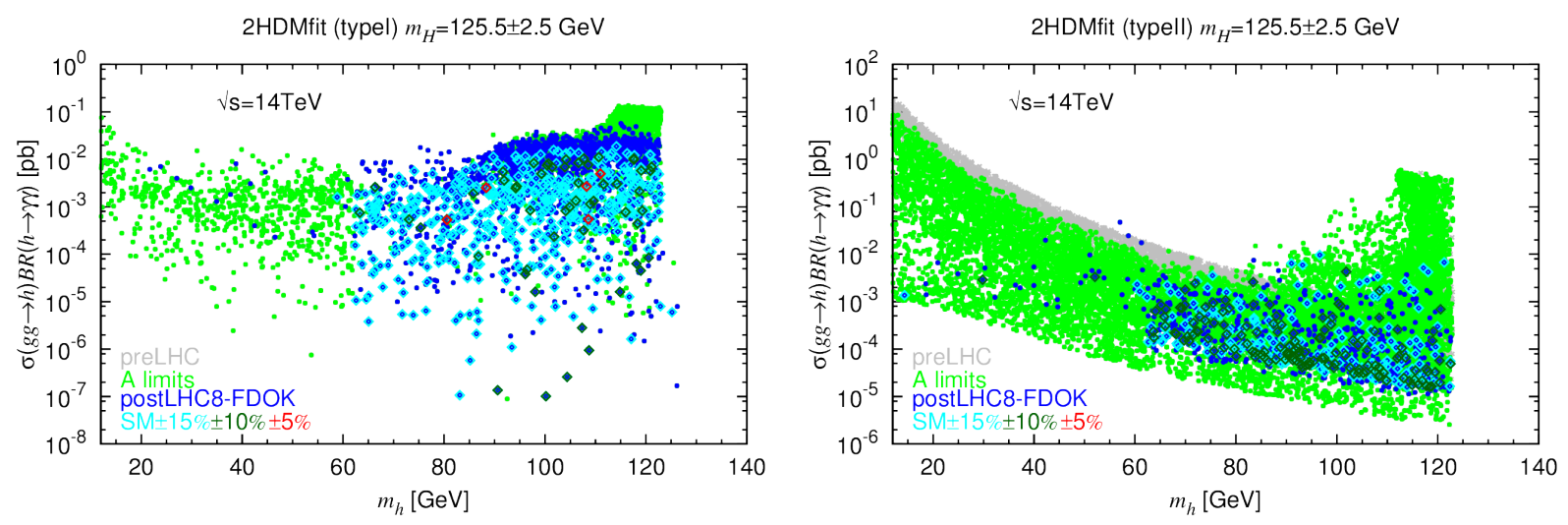

FIG. 26: $\sigma(g g \rightarrow h) \mathrm{BR}(h \rightarrow \gamma \gamma)$ for $\sqrt{s}=14 \mathrm{TeV}$ with postLHC8-FDOK constraints imposed as well as further limitations imposed by $\mathrm{SM} \pm 15 \%, \pm 10 \%, \pm 5 \%$ constraints. There are no FDOK requirements imposed on the preLHC and A-limits points. The $\mathrm{SM} \pm 15 \%, \pm 10 \%, \pm 5 \%$ points are subjected to FDOK requirements.

In the case of Type II models, $m_{A} \lesssim 200 \mathrm{GeV}$ is eliminated due to the $B$-physics limit of $m_{H^{ \pm}} \gtrsim 300 \mathrm{GeV}$ and the requirement of an acceptable $T$ parameter (which limits the $A-H^{ \pm}$ mass difference). In the case of Type I models, $m_{A} \lesssim 60 \mathrm{GeV}$ is possible but finding points with small enough $H \rightarrow A A$ to allow the $H$ to have reasonably SM-like properties requires significant fine-tuning. For most $m_{A}$, the Type II maximal and minimal cross sections tend to be substantially (by a factor of $>1000$ ) larger than for Type I. The lowest cross section values in Type I models are really very small at the largest allowed $m_{A}$ values and would not allow the detection of the $A$ boson. In contrast, in Type II models, even the very lowest cross section value of $\sim 5 \times 10^{-5}$ pb at $m_{A} \sim 630 \mathrm{GeV}$ would imply a handful of events for $L=300 \mathrm{fb}^{-1}$. The maximum Type II values imply a substantial number of events at all $m_{A}$, even at the largest masses, $m_{A} \sim 630 \mathrm{GeV}$. [We remind the reader that $630 \mathrm{GeV}$ is the upper limit allowed by perturbativity once the precision Higgs constraints, which limit $|\sin (\beta-\alpha)|$ to smaller values, have been included, cf. Fig. 20.]

As before, the rates for $A$ production in the $\mu \mu$ final state are simply obtained by the $\tan \beta$-independent rescaling factor $\operatorname{BR}(A \rightarrow \mu \mu) / \mathrm{BR}(A \rightarrow \tau \tau) \sim 3.5 \times 10^{-3}$. For $m_{A} \lesssim 2 m_{t}$, the cross section values near the upper limit obtained from such rescaling of the $\tau \tau$ final state rates shown in Fig. 27 are likely to be observable given the relatively narrow nature of the mass peak (typically of order a few $\mathrm{GeV}$ ) and the excellent $\mu \mu$ invariant mass resolution. 

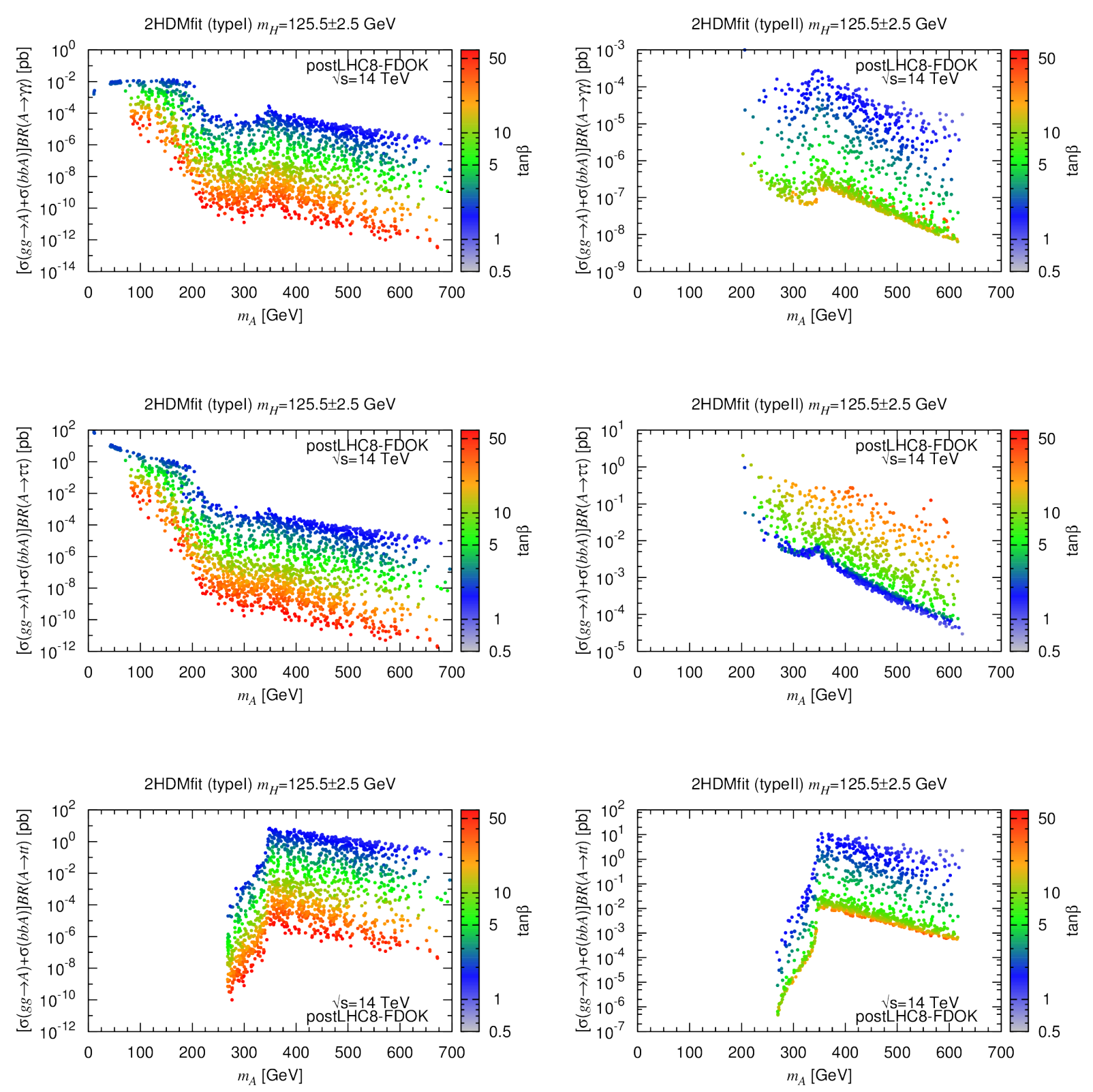

FIG. 27: Rates (in pb) of pseudoscalar $A$ production at $\sqrt{s}=14 \mathrm{TeV}$ as a function of $m_{A}$ for the $m_{H} \sim 125.5 \mathrm{GeV}$ scenarios, separated into different $A$ decay modes: $A \rightarrow \gamma \gamma$ (top), $A \rightarrow \tau \tau$ (middle) and $A \rightarrow t \bar{t}$ (bottom). In each case, we sum over $g g \rightarrow A$ and $g g \rightarrow b b A$ production. The values of $\tan \beta$ are color-coded as indicated by the scale on the right of the plots.

Last but not least, an interesting question is whether the $h, A$ (and $H^{ \pm}$) could all escape detection for some parameter choices when $m_{H} \sim 125.5 \mathrm{GeV}$. Given the upper limit discussed above of $m_{A} \lesssim 630 \mathrm{GeV}$ (after Higgs fitting constraints), a careful examination is 

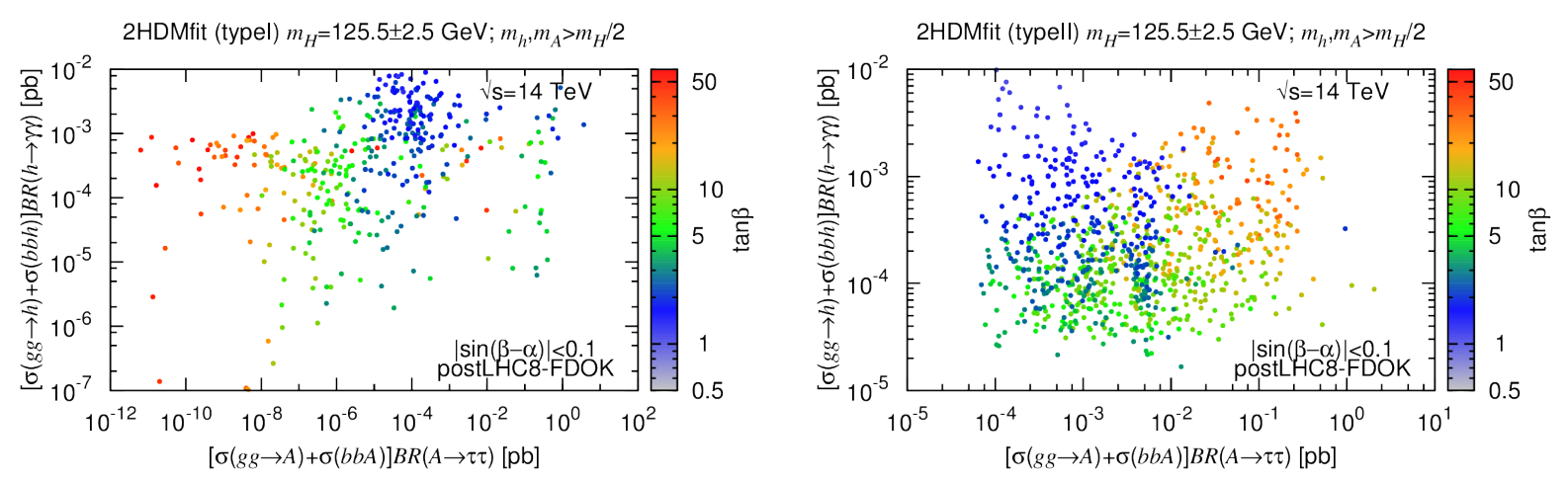

FIG. 28: We plot $[\sigma(g g \rightarrow h)+\sigma(b b h)] \operatorname{BR}(h \rightarrow \gamma \gamma)$ vs. $[\sigma(g g \rightarrow A)+\sigma(b b A)] \operatorname{BR}(A \rightarrow \tau \tau)$ for $\sqrt{s}=14 \mathrm{TeV}$ with postLHC8-FDOK constraints imposed. Colors indicate the value of $\tan \beta$. We have required $|\sin (\beta-\alpha)|<0.1$ so that the rate for $V^{*} \rightarrow V h$ will be at most 0.01 times the SM rate at the same mass.

required.

Consider first the $A$. In the case of Type I, Fig. 27 shows that for $m_{A} \in[200,300] \mathrm{GeV}$ (and most probably all the way out to the maximum allowed $m_{A}$ ) and large $\tan \beta$ the $\gamma \gamma, \tau \tau, t \bar{t}$ rates are all very small and would not allow discovery of the $A$ even with $L=$ $3000 \mathrm{fb}^{-1}$. In the case of Type II, the anticorrelation between the $\tau \tau$ and $t \bar{t}$ rates as $\tan \beta$ is varied, combined with the relatively substantial $\tau \tau$ rates for $m_{A}$ below the $t \bar{t}$ threshold combine to guarantee that the $A$ should be detectable with $L=3000 \mathrm{fb}^{-1}$ in either the $\tau \tau$ or the $t \bar{t}$ mode, and perhaps in both.

As regards the $h$, it is first of all clear that if $|\sin (\beta-\alpha)|$ is very small (as certainly both allowed and preferred by Higgs fitting) then the $V^{*} \rightarrow V h$ rates will be very tiny, as illustrated in Fig. 25 in the $m_{h}>60 \mathrm{GeV}$ region for both Type I and Type II. The other potentially viable mode is $g g \rightarrow h \rightarrow \gamma \gamma$. However, Fig. 26 shows very small rates in this case as well for Type I. In contrast, the lowest cross sections in this channel in Type II are of order $10^{-5} \mathrm{pb}$, a level which might be accessible with $L=3000 \mathrm{fb}^{-1}$.

To summarize the above, in Type II $m_{H} \sim 125.5 \mathrm{GeV}$ scenarios, the prospects for the discovery of the $A$ boson are very good, and there is an excellent chance of finding the $h$ as well in the $\gamma \gamma$ final state. In contrast, in Type I there are clearly regions of parameter space for which no Higgs boson other than the SM-like $H$ will be discoverable without going 
to still higher luminosity or energy. These results are summarized in Fig. 28 where we see from the right-hand plot that in Type II both the $h$ and $A$ have an excellent chance of being detectable in the $\gamma \gamma$ and $\tau \tau$ modes, respectively. In contrast, the left-hand plot shows that there is clearly a corner of parameter space where detection of both the $h$ and the $A$ will be extremely challenging. Of course, we have not explored prospects for $H^{ \pm}$discovery in this paper, but its production cross sections are typically substantially below those for the $A$ since $H^{ \pm}$production requires at least one top quark in the final state.

Finally, we note the regions with $m_{A} \sim 200-350 \mathrm{GeV}$ and low $\tan \beta$ of the $\gamma \gamma$ and $\tau \tau$ final state Type I plots that have been depleted by the removal of points with excessive feed down. These regions are, of course, the analogue of the FD-depleted regions discussed in the case of $m_{h} \sim 125.5 \mathrm{GeV}$.

\section{CONCLUSIONS}

The latest Higgs data from the LHC clearly favor a fairly SM-like Higgs boson with mass of about $125.5 \mathrm{GeV}$. In this paper we have quantified this in the context of Type I and Type II 2HDMs, identifying either the $h$ or the $H$ as the $125.5 \mathrm{GeV}$ state. Indeed, the vector boson pair coupling of the $125.5 \mathrm{GeV}$ state must be quite close to the SM Higgs coupling, but at low $\tan \beta$ there is significant dispersion about the SM values for the fermionic couplings. Given the constraints on the $125.5 \mathrm{GeV}$ state, we have ascertained expectations for the other Higgs bosons $H, H^{ \pm}$and $A$ in the case that it is the $h$ that gives the SM-like signal. We demonstrated that there are many parameter space points (satisfying all $95 \%$ C.L. constraints on the $h$ ) that for $\sqrt{s}=14 \mathrm{TeV}$ could allow for observation of the $H$ and $A$ in a variety of modes, including not just the $Z Z$ (for $H$ only), $\tau \tau$ and $t \bar{t}$ final states, but also $\mu \mu$ and $\gamma \gamma$. In the $m_{H} \sim 125.5 \mathrm{GeV}$ case, the $A$ can also be detected in these same modes (except $Z Z$ ). In addition, there is good probability for viable signals for the lighter $h$. In particular, the $V^{*} \rightarrow V h$ with $h \rightarrow b \bar{b}$ channel might yield a detectable signal for at least a fraction of the points surviving the $95 \%$ C.L. limits on the $H$ signal at $125.5 \mathrm{GeV}$.

Nondecoupling of the charged-Higgs loop contribution to the $h \gamma \gamma$ or $H \gamma \gamma$ coupling plays an important role once rates are required to be within $\pm 5 \%$ of the $\mathrm{SM}$ predictions. The result is elimination of all but a tiny fraction of the $m_{H} \sim 125.5 \mathrm{GeV}$ Type I scenarios and all of the $m_{H} \sim 125.5 \mathrm{GeV}$ Type II scenarios as well as all of the wrong-sign Yukawa Type II 
scenarios in the $m_{h} \sim 125.5 \mathrm{GeV}$ case.

Along the way, we clarified the important role played by constraints deriving from requirements of perturbativity in reducing the otherwise huge parameter space of the 2HDMs. We also delineated the (fortunately) rather limited impact of removing points that suffer from a large amount of feed down from heavier Higgs bosons decaying either directly or via chain decay to a final state containing the $125.5 \mathrm{GeV}$ state.

An important general conclusion is that even if the $125.5 \mathrm{GeV}$ signal rates converge to very SM-like values, the $2 \mathrm{HDM}$ s predict ample opportunity for detecting the other Higgs bosons. Still, it is also true that in the case of $m_{h} \sim 125.5 \mathrm{GeV}$ in the decoupling limit of very large $m_{A} \sim m_{H} \sim m_{H^{ \pm}}$detection of even one of the other Higgs bosons at LHC14 would not be possible. However, the case of $m_{H} \sim 125.5 \mathrm{GeV}$ is different. Because the maximum $m_{A}$ is limited, having the $h, A$ and $H^{ \pm}$all escape detection is very unlikely in Type II 2HDM. However, in Type I if $m_{A} \in[200,300] \mathrm{GeV}$ and $\tan \beta$ is large, then the $A$ will not be detectable and $h$ detection will be very marginal.

Finally, we make special note of the scenarios with low $m_{A}<100 \mathrm{GeV}$ that escape all LEP and (so far) LHC limits and yet have quite substantial $g g \rightarrow A$ and $b b A$ production cross sections. It will be interesting to probe these scenarios, which are possible for both Type I and Type II in the $m_{h} \sim 125.5 \mathrm{GeV}$ case and for Type I in the $m_{H} \sim 125.5 \mathrm{GeV}$ case, in ongoing analyses of LHC $8 \mathrm{TeV}$ data and in future LHC running at higher energy.

\section{ACKNOWLEDGEMENTS}

This work was supported in part by U.S. DOE Grant No. DE-SC-000999 and by IN2P3 under PICS FR-USA Contract No. 5872. Y.J. is also supported by LHC-TI fellowship US NSF Grant No. PHY-0969510. In addition, he thanks the "Investissements davenir, Labex ENIGMASS" for partial financial support for a research stay at LPSC Grenoble. J.F.G. and S.K. thank the Aspen Center for Physics, supported by the National Science Foundation under Grant No. PHYS-1066293, for hospitality. 


\section{Appendix A: Generic formalism for feed down processes}

We have quantitatively computed feed down as follows. Let us define the final state of interest by $Y=\gamma \gamma, V V, b \bar{b}, \tau \tau$ (the only final states quantitatively of relevance for fitting the $\sim 125.5 \mathrm{GeV}$ signal using the $7+8 \mathrm{TeV}$ data set). The production processes will be denoted by $\mathrm{XH}=\mathrm{ggFH}$ (standing for $g g \rightarrow \mathrm{H}$ ), bbH (standing for associated production of the $\mathrm{H}$ with a $b \bar{b}$ pair), ttH, VBFH (standing for $W W / Z Z \rightarrow \mathrm{H}$ ), and $\mathrm{VH}$. (Note that the regular Roman $\mathrm{H}$ is generic and we will be using these production processes for various different choices of H.) In this paper, we will consider ggFH and bbH together and compute their combined feed down. We wish to consider the feed down into $\mathbf{H}=h$ and $H$ where $\mathbf{H}$ is always the $125.5 \mathrm{GeV}$ state. In the process, we will have to consider production of all Higgses that are heavier than the $\mathbf{H}$, which Higgses we will denote by $\mathcal{H}$.

For all but VH, we can define the fraction of feed down contributing to production mode $X$ as

$$
\mu_{X \mathbf{H}}^{\mathrm{FD}} \equiv \frac{\sum_{\mathcal{H}} \sigma_{X \mathcal{H}} P_{\mathrm{FD}}(\mathcal{H} \rightarrow \mathbf{H}+\text { anything })}{\sigma_{X \mathbf{H}}} .
$$

Note that we have cancelled a common factor of $\mathrm{BR}(\mathbf{H} \rightarrow Y)$, the branching ratio for $\mathbf{H}$ to decay to any final state $Y=\gamma \gamma, V V, b \bar{b}, \tau \tau$, that is common to the numerator and denominator. In the above, we have to take into account the possibilities that the intermediating $\mathcal{H}$ can decay to two $\mathbf{H}$ 's or only one $\mathbf{H}$. Thus, we actually have

$$
P_{\mathrm{FD}}(\mathcal{H} \rightarrow \mathbf{H}+\text { anything })=2 P_{\mathcal{H}, 2 \mathbf{H}}+P_{\mathcal{H}, 1 \mathbf{H}}
$$

so that in the first case the "anything" includes the 2 nd $\mathbf{H}$. For $2 \mathbf{H}$ final states we include a multiplicative factor of 2 since both of the final $\mathbf{H}$ 's will contribute. The formulas for the various $P_{\mathcal{H}, 2 \mathbf{H}}$ and $P_{\mathcal{H}, 1 \mathbf{H}}$ 's cases can be found in [31] which we will repeat in the following context (including some corrections we found).

The VH process must be handled differently due to the fact that it is directly impacted by $g g \rightarrow A$ and $b b A$ production with $A \rightarrow Z \mathbf{H}$ in the case of $V=Z$ and by $g g \rightarrow t b H^{ \pm} \rightarrow$ $t b W^{ \pm} \mathbf{H}$ in the case of $V=W$. The largest of these fractional contaminations is that associated with $g g \rightarrow A+b b A$ since the cross sections for $H^{ \pm}$production are quite a bit smaller. In the $V=Z$ case, the leading contribution to the fractional feed down is

$$
\mu_{Z \mathbf{H}}^{\mathrm{FD}}=\frac{\left[\sigma_{\mathrm{ggF} A}+\sigma_{\mathrm{bb} A}\right] \mathrm{BR}(A \rightarrow Z \mathbf{H})}{\sigma_{\mathrm{ZH}}},
$$


where the $\mathrm{BR}(\mathbf{H} \rightarrow Y)$ branching ratios in numerator and denominator have once again cancelled and $\sigma_{\mathbf{Z H}}$ is that arising from the $Z^{*} \rightarrow Z \mathbf{H}$ subprocess. In the above, we have neglected (a good approximation) contributions to the $Z \mathbf{H}+$ anything final state due to the FD processes other than the direct $g g \rightarrow A \rightarrow Z \mathbf{H}$ production process. In particular, we neglect the terms which lead to final states containing $Z \mathbf{H}$ plus additional particles. Besides yielding small contributions to $\mu_{V \mathbf{H}}^{\mathrm{FD}}$, such terms yield final states that are much more complicated than the simple $Z \mathbf{H}$ final state, containing, for example, more than one $Z$ or a $Z+W$ pair. The selection criteria for the $Z \mathbf{H}$ final state are likely to be such that these more complicated final states were discarded or strongly discriminated against. In the following subsections, we apply these general formulas to the two 2HDM scenarios discussed in this paper, showing how to determine the magnitude of feed down associated with a given point in parameter space.

\section{Example I: Feed down of heavier Higgs bosons to a $125.5 \mathrm{GeV} h$}

In this case, the $\mathbf{H}=h$ can be produced from the chain decay of heavier Higgs bosons $\mathcal{H}=H, A, H^{ \pm}$. Since the cross section for $g g \rightarrow t b H^{ \pm}$is relatively smaller than those for $H$ and $A$ (because of the $t$ in the $t b H^{ \pm}$final state), we will not include $H^{ \pm}$as an intermediate source for the FD mechanism. In practice, important FD contributions arise from $(\mathrm{ggF}+\mathrm{bb}) H,(\mathrm{ggF}+\mathrm{bb}) A$ or $\mathrm{VBF} H$. The most important feed down decays for $g g \mathrm{~F}+\mathrm{bb}$ are $H \rightarrow h h$ and $A \rightarrow Z h$ (or $A \rightarrow Z H$ for the case of $m_{H} \sim 125.5 \mathrm{GeV}$ ) and for VBF $H \rightarrow h h$. However, the approximations of

$$
P_{H, 2 h} \simeq \mathrm{BR}(H \rightarrow h h), \quad P_{A, 1 h} \simeq \mathrm{BR}(A \rightarrow Z h)
$$

are not generally adequate.

It is convenient to consider separately the chains that can yield $2 h$ in the final state vs. those that yield only $1 h$ in the final state. Some sample net branching ratios, denoted (following [31]) by $P$ are the following. First, if $H$ is the primary then $H \rightarrow A A$ and 
$H \rightarrow H^{+} H^{-}$are possible for some $2 \mathrm{HDM}$ points in addition to $H \rightarrow h h$. Thus, we have

$$
\begin{aligned}
P_{H, 2 h}= & \mathrm{BR}(H \rightarrow h h)+\mathrm{BR}\left(H \rightarrow H^{+} H^{-}\right)\left[\mathrm{BR}\left(H^{+} \rightarrow W^{+} h\right)^{2}\right. \\
& +\mathrm{BR}\left(H^{+} \rightarrow W^{+} A\right)^{2} \mathrm{BR}(A \rightarrow Z h)^{2} \\
& \left.+2 \mathrm{BR}\left(H^{+} \rightarrow W^{+} h\right) \mathrm{BR}\left(H^{+} \rightarrow W^{+} A\right) \mathrm{BR}(A \rightarrow Z h)\right] \\
& +\mathrm{BR}(H \rightarrow A A)\left[\mathrm{BR}(A \rightarrow Z h)^{2}+4 \mathrm{BR}\left(A \rightarrow W^{-} H^{+}\right)^{2} \mathrm{BR}\left(H^{+} \rightarrow W^{+} h\right)^{2}\right. \\
& \left.+4 \mathrm{BR}(A \rightarrow Z h) \mathrm{BR}\left(A \rightarrow W^{-} H^{+}\right) \mathrm{BR}\left(H^{+} \rightarrow W^{+} h\right)\right] \\
P_{H, 1 h}= & \mathrm{BR}(H \rightarrow Z A) \operatorname{BR}(A \rightarrow Z h)+2 \mathrm{BR}\left(H \rightarrow W^{-} H^{+}\right) \mathrm{BR}\left(H^{+} \rightarrow W^{+} h\right) \\
+ & 2 \mathrm{BR}\left(H \rightarrow H^{+} H^{-}\right)\left[\mathrm{BR}\left(H^{+} \rightarrow W^{+} h\right)\right. \\
+ & \left.\mathrm{BR}\left(H^{+} \rightarrow W^{+} A\right) \operatorname{BR}(A \rightarrow Z h)\right] p\left(H^{+} \not \rightarrow h\right) \\
+ & \mathrm{BR}(H \rightarrow A A)\left[2 \mathrm{BR}(A \rightarrow Z h)+4 \mathrm{BR}\left(A \rightarrow W^{-} H^{+}\right) \mathrm{BR}\left(H^{+} \rightarrow W^{+} h\right)\right] p(A \not A h)
\end{aligned}
$$

where

$$
\begin{gathered}
p\left(H^{+} \not \rightarrow h\right)=1-\mathrm{BR}\left(H^{+} \rightarrow W^{+} h\right)-\mathrm{BR}\left(H^{+} \rightarrow W^{+} A\right) \mathrm{BR}(A \rightarrow Z h), \\
p(A \not \rightarrow h)=1-\mathrm{BR}(A \rightarrow Z h)-2 \mathrm{BR}\left(A \rightarrow W^{-} H^{+}\right) \mathrm{BR}\left(H^{+} \rightarrow W^{+} h\right) .
\end{gathered}
$$

If $A$ is the primary then possibly accessible modes at the first stage are $A \rightarrow Z H$, and $A \rightarrow W^{+} H^{-}, W^{-} H^{+}$, resulting in

$$
\begin{aligned}
P_{A, 2 h} & =\mathrm{BR}(A \rightarrow Z H) \bar{P}_{H, 2 h}+2 \mathrm{BR}\left(A \rightarrow W^{-} H^{+}\right) \mathrm{BR}\left(H^{+} \rightarrow W^{+} H\right) \mathrm{BR}(H \rightarrow h h), \\
P_{A, 1 h} & =\mathrm{BR}(A \rightarrow Z h)+\mathrm{BR}(A \rightarrow Z H) \bar{P}_{H, 1 h} \\
& +2 \mathrm{BR}\left(A \rightarrow W^{-} H^{+}\right) \mathrm{BR}\left(H^{+} \rightarrow W^{+} h\right),
\end{aligned}
$$

where the appropriate expressions for the two $P$ 's are the same as given earlier with the $A$ channels eliminated since $H$ being a secondary particle must be lighter than the $A$.

$$
\begin{aligned}
\bar{P}_{H, 2 h} & =\operatorname{BR}(H \rightarrow h h)+\mathrm{BR}\left(H \rightarrow H^{+} H^{-}\right) \mathrm{BR}\left(H^{+} \rightarrow W^{+} h\right)^{2}, \\
\bar{P}_{H, 1 h} & =2 \mathrm{BR}\left(H \rightarrow W^{-} H^{+}\right) \mathrm{BR}\left(H^{+} \rightarrow W^{+} h\right) \\
& +2 \mathrm{BR}\left(H \rightarrow H^{+} H^{-}\right) \operatorname{BR}\left(H^{+} \rightarrow W^{+} h\right) p\left(H^{+} \not \rightarrow h\right) .
\end{aligned}
$$

For the sake of completeness, we also provide the formula for the case that $\mathrm{H}^{+}$or $\mathrm{H}^{-}$is produced as the primary Higgs. In this case, the first level decays of relevance are $H^{+} \rightarrow$ 
$W^{+} h$ and $H^{+} \rightarrow W^{+} H$, leading to

$$
\begin{aligned}
& P_{H^{+}, 2 h}=\mathrm{BR}\left(H^{+} \rightarrow W^{+} H\right) P_{H, 2 h}+\mathrm{BR}\left(H^{+} \rightarrow W^{+} A\right) \mathrm{BR}(A \rightarrow Z H) \mathrm{BR}(H \rightarrow h h), \quad(\mathrm{A} 10) \\
& P_{H^{+}, 1 h}=\mathrm{BR}\left(H^{+} \rightarrow W^{+} h\right)+\mathrm{BR}\left(H^{+} \rightarrow W^{+} H\right) P_{H, 1 h}+\mathrm{BR}\left(H^{+} \rightarrow W^{+} A\right) \operatorname{BR}(A \rightarrow Z h),
\end{aligned}
$$

where the $P_{H, 2 h}$ and $P_{H, 1 h}$ are as given earlier in Eqs. A5 and (A6), respectively, with kinematics for the $H^{+}$primary situation eliminating the terms involving $H \rightarrow W^{-} H^{+}$and $H \rightarrow H^{+} H^{-}$.

Furthermore, in order to illustrate the importance of keeping the full formulas we show in Fig. 29 the fraction of $1 h$ final states coming directly from $A \rightarrow Z h$ vs. the fraction of $2 h$ final states coming directly from $H \rightarrow h h$.
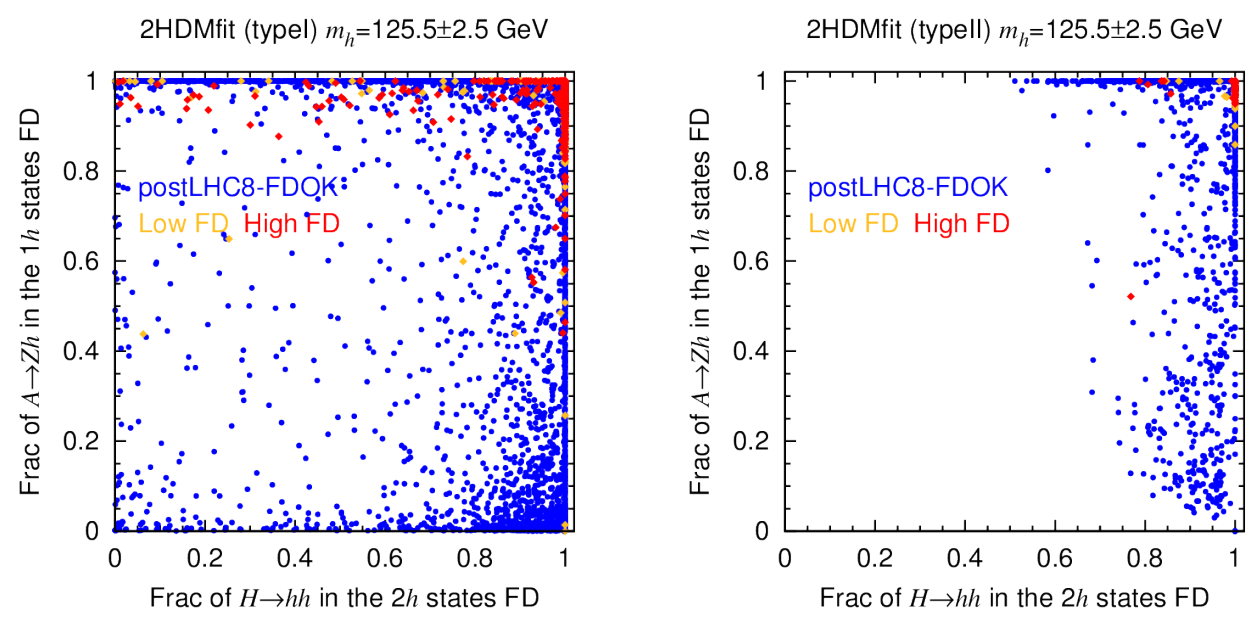

FIG. 29: We plot $[\sigma(g g \rightarrow H+b b H) 2 \mathrm{BR}(H \rightarrow h h)] /\left[\sigma(g g \rightarrow H+b b H) 2 P_{H, 2 h}+\sigma(g g \rightarrow A+\right.$ $\left.b b A) 2 P_{A, 2 h}\right]$ on the $x$ axis and $[\sigma(g g \rightarrow A+b b A) \mathrm{BR}(A \rightarrow Z h)] /\left[\sigma(g g \rightarrow H+b b H) P_{H, 1 h}+\sigma(g g \rightarrow\right.$ $\left.A+b b A) P_{A, 1 h}\right]$ on the $y$ axis. The postLHC8-FDOK points are displayed in blue. The cyan, green and red points obey FDOK constraints and have all the channel rates of Eq. (6) within $\mathrm{SM} \pm 15 \%$, $\mathrm{SM} \pm 10 \%$ and $\mathrm{SM} \pm 5 \%$, respectively; cf. Fig. 7 .

\section{Example II: Feed down of heavier Higgs to a $125.5 \mathrm{GeV} H$}

The case of $\mathbf{H}=H$ in the $2 \mathrm{HDM}$ is much simpler. Ignoring the $H^{ \pm}$as explained above, the only heavier Higgs which can feed the $H$ signal is $\mathcal{H}=A$. Therefore, the most important FD processes are $g g \rightarrow A+b b A$ with $A \rightarrow Z H$. However, the approximations of

$$
P_{A, 1 H} \simeq \mathrm{BR}(A \rightarrow Z H)
$$


are not generally applicable. In order to measure the FD precisely, one should use the full expression for $P_{A, 2 H}$ and $P_{A, 1 H}$ as follows

$$
\begin{aligned}
P_{A, 2 H} & =\operatorname{BR}\left(A \rightarrow H^{+} H^{-}\right) \operatorname{BR}\left(H^{+} \rightarrow W^{+} H\right)^{2}, \\
P_{A, 1 H} & =\operatorname{BR}(A \rightarrow Z H)+2 \operatorname{BR}\left(A \rightarrow W^{-} H^{+}\right) \operatorname{BR}\left(H^{+} \rightarrow W^{+} H\right) \\
& +2 \operatorname{BR}\left(A \rightarrow H^{+} H^{-}\right) \operatorname{BR}\left(H^{+} \rightarrow W^{+} H\right) p\left(H^{+} \not \rightarrow H\right),
\end{aligned}
$$

where

$$
p\left(H^{+} \not \rightarrow H\right)=1-\mathrm{BR}\left(H^{+} \rightarrow W^{+} H\right) .
$$

\section{Appendix B: Nondecoupling of the $H^{ \pm}$loop contribution to the $H \gamma \gamma$ coupling and wrong-sign Yukawa effects on the $g g H$ coupling.}

We have claimed that the $m_{H}=125.5 \mathrm{GeV}$ Type II scenarios can be either eliminated or confirmed when the LHC measurements reach a precision such that the rates in the various initial $\times$ final state channels can be measured to $5 \%$ accuracy. As we have said, this is because for this scenario the charged Higgs loop does not decouple in the $H \gamma \gamma$ coupling calculation and results in at least a $5 \%$ reduction in the coupling relative to the SM prediction, i.e. in $C_{\gamma}^{H}$. This can easily be explained using the results of Appendix B of [41] as translated to the current situation.

We first emphasize that in all the fits, whether Type I or Type II, the LHC data require that the sign of the top-quark Yukawa, i.e. $C_{U}^{H}$, be the same as the sign of the $H V V$ coupling $C_{V}^{H}$ in order that the $\gamma \gamma$ final state rates not be too enhanced. Referring to Table If we see that a SM-like $H$ results in $\cos (\beta-\alpha)= \pm 1$, for which $C_{U}^{H}= \pm 1$, respectively. In our scan range of $|\alpha| \leq \pi / 2$ both signs are found. For Type I, $C_{U}^{H}$ and $C_{D}^{H}$ have the same sign. For Type II, $C_{D}^{H}$ is always positive within the $\alpha$ scan range. Thus, in Type I we always have the same relative signs between all the couplings as for a SM Higgs boson, whereas in Type II there will be scan points for which $C_{D}^{H}$ has the "wrong" sign relative to $C_{V}^{H}$ and $C_{U}^{H}$. This will impact the $g g H$ 1-loop coupling because the interference between the top-quark and bottom-quark loops will change sign, thereby increasing the $g g H$ coupling by a factor of $C_{g}^{H} \sim 1.12$ relative to the SM value - see Appendix B of [41] - a level that can be probed in a future LHC run from the observation of the decay products of Higgs bosons originating from gluon fusion. In contrast, the change in sign of the bottom-quark loop contribution to 
the $H \gamma \gamma$ coupling yields a $\lesssim 1 \%$ decrease in $C_{\gamma}^{H}$ due to the dominance of the $W$ - and $t$-loop contributions. However, it turns out that for all of the Type II scenarios the charged-Higgs loop contribution to the $H \gamma \gamma$ coupling does not decouple as $m_{H^{ \pm}}$becomes large. As a result, all Type II scenarios with $m_{H} \sim 125.5 \mathrm{GeV}$ will be eliminated if future LHC data determine that $C_{\gamma}^{H}$ is within $5 \%$ of the SM prediction of unity. This happens regardless of the common sign of $C_{V}^{H}$ and $C_{U}^{H}$, denoted by $\operatorname{sgn}\left(C_{V}^{H}\right)$.

To gain intuition, we employ the results of [7] to find that in the limit where $\cos (\beta-\alpha) \rightarrow$ \pm 1 the dimensionless $\mathrm{HH}^{+} \mathrm{H}^{-}$coupling takes the form

$$
g_{H H^{+} H^{-}}=-\frac{\operatorname{sgn}\left(C_{V}^{H}\right)}{v^{2}}\left(m_{H}^{2}+2 m_{H^{ \pm}}^{2}-2 \hat{m}_{12}^{2}\right) .
$$

Further, perturbativity requires that $\hat{m}_{12}^{2}$ be of modest size, and, of course, $m_{H}$ is fixed at $\sim 125.5 \mathrm{GeV}$. Thus, we have the asymptotic result that

$$
\frac{v^{2} g_{H H^{+} H^{-}} \operatorname{sgn}\left(C_{V}^{H}\right)}{m_{H^{ \pm}}^{2}} \rightarrow-2 .
$$

As shown in Appendix B of [41] for the case of $C_{V}^{H}>0$ (but the same arguments apply with all signs changed to the $C_{V}^{H}<0 \operatorname{case}^{8}$ ), this means that $C_{\gamma}^{H}$ is reduced by $5 \%$ relative to the SM value of unity. Of course, $\hat{m}_{12}$ is not exactly zero, but one finds that $\hat{m}_{12}^{2}$ lies below $(100 \mathrm{GeV})^{2}$ while being able to reach large negative values for a large range of $m_{H^{ \pm}}$. The result is that $v^{2} g_{H H^{+} H^{-}} \operatorname{sgn}\left(C_{V}^{H}\right)$ is always at most $-2 m_{H^{ \pm}}^{2}$, implying that the decrease in $C_{\gamma}^{H}$ is at least $5 \%$. This is illustrated in Fig. 30 .

This Type II situation can be contrasted with Type I. As stated earlier, in Type I $C_{V}^{H}$, $C_{U}^{H}$ and $C_{D}^{H}$ all have the same sign, which can be either plus or minus with respect to the SM convention of $C_{V}^{H_{\mathrm{SM}}}=+1$. We plot in Fig. 31 the same quantities as in Fig. 30. We observe that the bulk of the parameter space, namely that portion with $C_{D}^{H}>0$, has $v^{2} g_{H H^{+} H^{-}} \operatorname{sgn}\left(C_{V}^{H}\right) \lesssim-2 m_{H^{ \pm}}^{2}$ leading to at least a $5 \%$ suppression of $C_{\gamma}$. In the other branch, we have $C_{D}^{H}<0$. In the end, various effects are competing and $v^{2} g_{H H^{+} H^{-}} \operatorname{sgn}\left(C_{V}^{H}\right) \gtrsim$ $-2 m_{H^{ \pm}}^{2}$, with values near zero and positive values as well being possible. The result is that $C_{\gamma}^{H} \sim 1$ is achievable and a good fit to LHC data is possible, although quite rare within our scans.

${ }^{8}$ The $b$-quark loop, which does not change sign when $C_{V}^{H}<0$, is negligible relative to other contributions. 

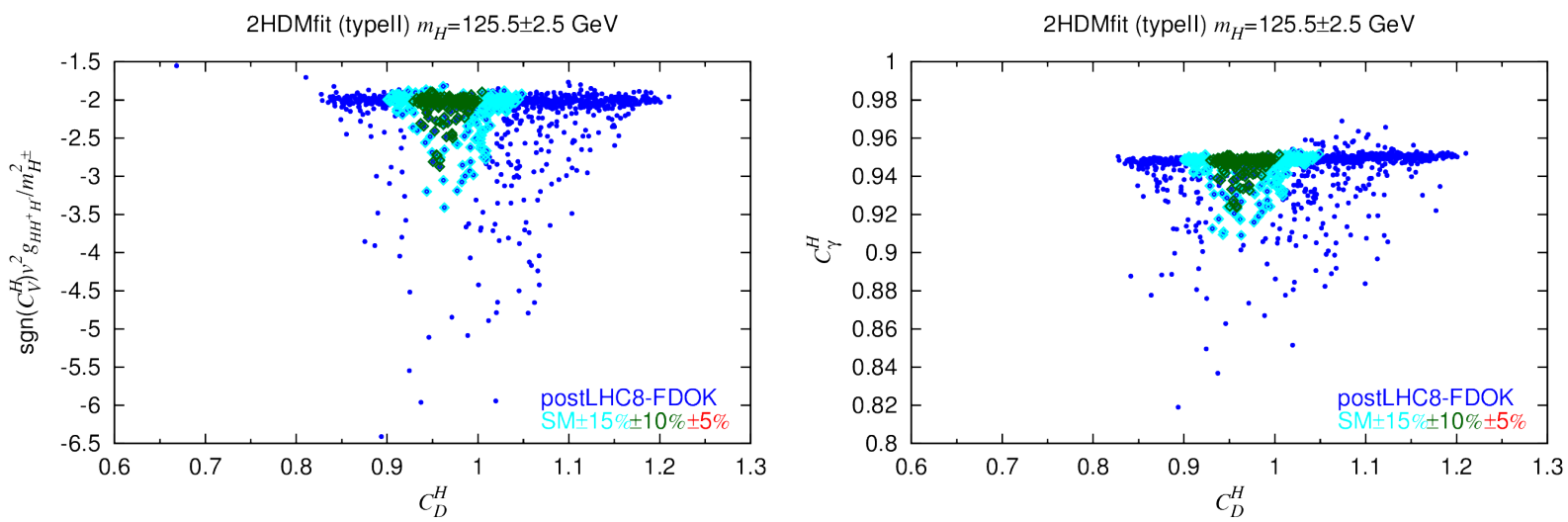

FIG. 30: For Type II $2 \mathrm{HDM}$, we plot: $v^{2} g_{H H^{+} H^{-}} \operatorname{sgn}\left(C_{V}^{H}\right) / m_{H^{ \pm}}^{2}$ vs. $C_{D}^{H}$ (left) and $C_{\gamma}^{H}$ vs. $C_{D}^{H}$ (right). We show FDOK points consistent at the postLHC8 level (blue) and after presumed LHC measurements showing SM consistency at the $\pm 15 \%$ (cyan) and $\pm 10 \%$ (dark green) level. No points survive if SM consistency is demonstrated at the $\pm 5 \%$ level.
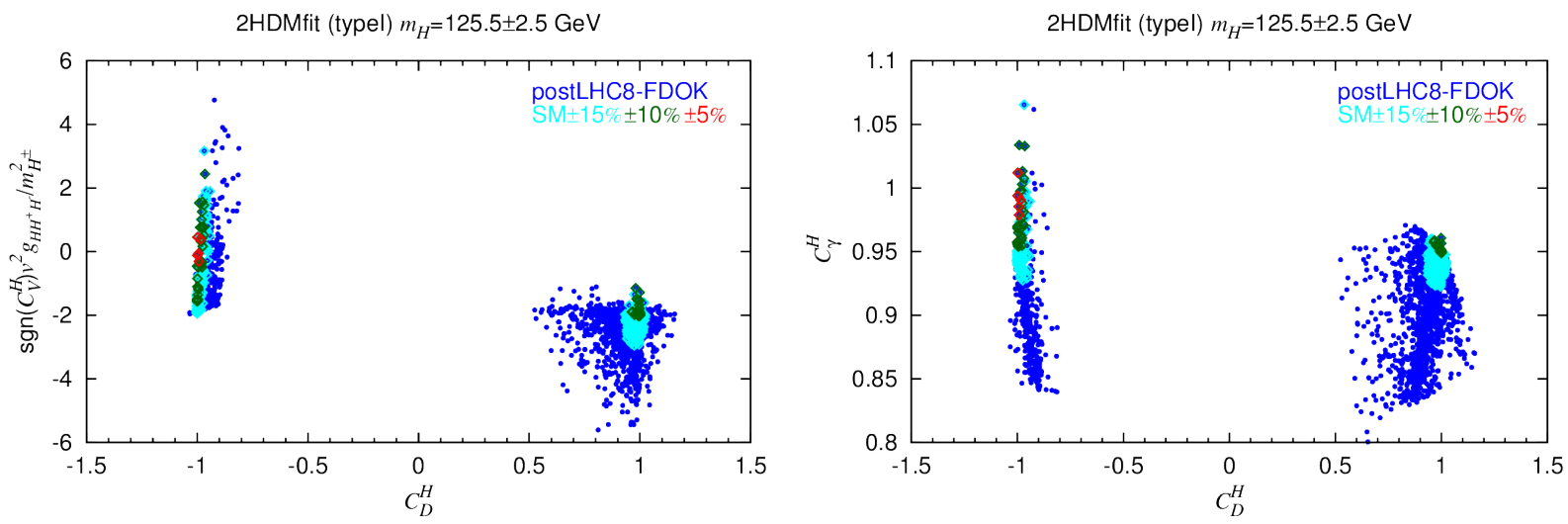

FIG. 31: For Type I 2HDM, we plot: Left, $v^{2} g_{H H^{+} H^{-}} \operatorname{sgn}\left(C_{V}^{H}\right) / m_{H^{ \pm}}^{2}$ vs. $C_{D}^{H}$ (left) and $C_{\gamma}^{H}$ vs. $C_{D}^{H}$ (right), showing FDOK points consistent at the postLHC8 level (blue) and after presumed LHC measurements showing SM consistency at the $\pm 15 \%$ (cyan), $\pm 10 \%$ (dark green) and $\pm 5 \%$ (red) levels.

A second means of discrimination relative to the SM arises in the context of the $g g H$ coupling in the case of a Type II model. As noted above, in Type II $C_{D}^{H}$ always has the same sign as in the SM, but $C_{U}^{H}$ can have either the SM sign or a sign opposite the SM, i.e. $C_{U}^{H} \sim-1$. In this latter case, the sign of the interference terms between the top-quark and bottom-quark loop contributions to the $g g H$ coupling changes. This results in a significant 
increase of about $12 \%$ in the $g g H$ coupling, as detailed in [41]. As also discussed there, $C_{g}^{H}$ can eventually be measured to an accuracy of about $5 \%$ in future LHC runs as well as at a future linear collider, which would allow either confirmation or conflict with the $C_{U}^{H} \sim-1$ Type II case. In Fig. 32, we plot $C_{g}^{H}$ as a function of $C_{U}^{H}$ for both Type I and Type II models. There, we see that $C_{g}^{H}$ is not generally a useful discriminator in the case of the Type I model nor on the $C_{U}^{H} \sim+1$ branch in the case of a Type II model.
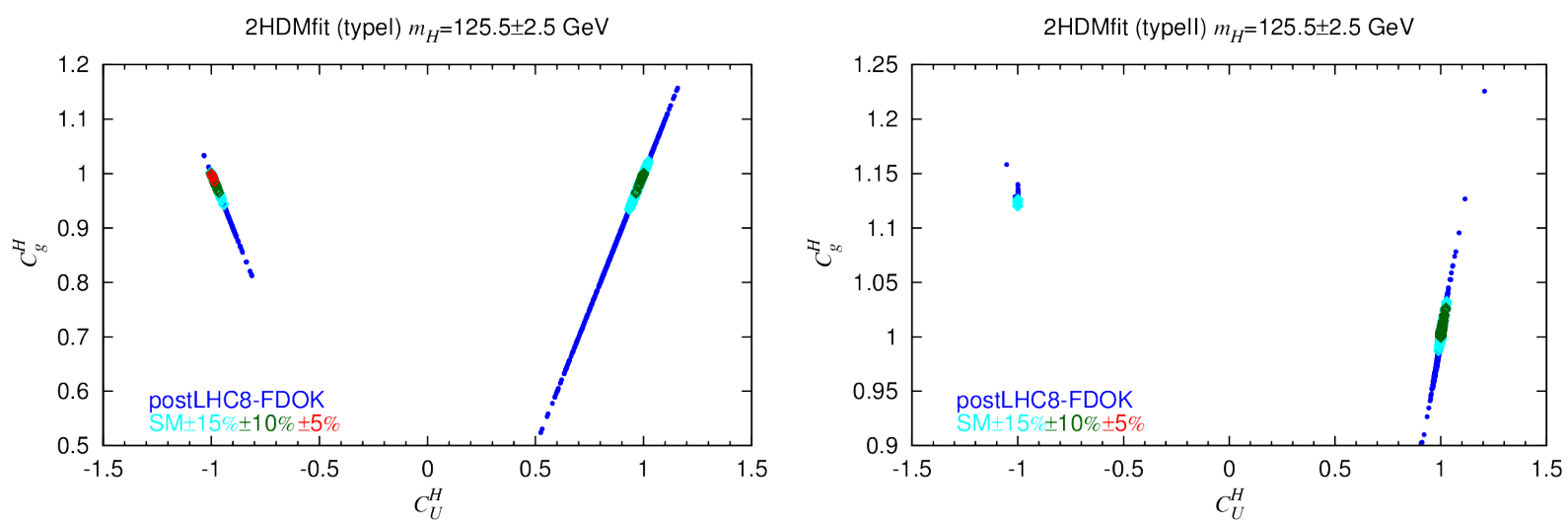

FIG. 32: We plot $C_{g}^{H}$ vs. $C_{U}^{H}$ for Type I (right) and Type II (left). We show FDOK points consistent at the postLHC8 level (blue) and after presumed LHC measurements showing SM consistency at the $\pm 15 \%$ (cyan) and $\pm 10 \%$ (dark green) levels. Points consistent with the SM (red points) at the $\pm 5 \%$ level are only present in the $C_{U}^{H}<0$ branch of the Type I model. 
[1] ATLAS Collaboration, G. Aad et al., Observation of a new particle in the search for the Standard Model Higgs boson with the ATLAS detector at the LHC, Phys.Lett. B716 (2012) 1-29, $\operatorname{arXiv:1207.7214.}$

[2] CMS Collaboration, S. Chatrchyan et al., Observation of a new boson at a mass of 125 GeV with the CMS experiment at the LHC, Phys.Lett. B716 (2012) 30-61, arXiv:1207.7235.

[3] ATLAS Collaboration, G. Aad et al., Updated coupling measurements of the Higgs boson with the ATLAS detector using up to 25/fb of proton-proton collision data, 2014. ATLAS-CONF-2014-009.

[4] CMS Collaboration, Combination of standard model Higgs boson searches and measurements of the properties of the new boson with a mass near 125 GeV, 2013. CMS PAS HIG-13-005.

[5] ATLAS Collaboration, G. Aad et al., Measurements of Higgs boson production and couplings in diboson final states with the ATLAS detector at the LHC, Phys.Lett. B726 (2013) 88-119, arXiv:1307.1427.

[6] J. F. Gunion, H. E. Haber, G. L. Kane, and S. Dawson, THE HIGGS HUNTER'S GUIDE, Front.Phys. 80 (2000) 1-448.

[7] J. F. Gunion and H. E. Haber, The CP conserving two Higgs doublet model: The Approach to the decoupling limit, Phys.Rev. D67 (2003) 075019, hep-ph/0207010.

[8] G. Branco, P. Ferreira, L. Lavoura, M. Rebelo, M. Sher, et al., Theory and phenomenology of two-Higgs-doublet models, Phys.Rept. 516 (2012) 1-102, arXiv:1106.0034.

[9] C.-W. Chiang and K. Yagyu, Implications of Higgs boson search data on the two-Higgs doublet models with a softly broken $Z_{2}$ symmetry, JHEP 1307 (2013) 160, arXiv:1303.0168.

[10] B. Grinstein and P. Uttayarat, Carving Out Parameter Space in Type-II Two Higgs Doublets Model, JHEP 1306 (2013) 094, arXiv:1304.0028.

[11] B. Coleppa, F. Kling, and S. Su, Constraining Type II 2HDM in Light of LHC Higgs Searches, JHEP 1401 (2014) 161, [arXiv:1305.0002].

[12] O. Eberhardt, U. Nierste, and M. Wiebusch, Status of the two-Higgs-doublet model of type II, JHEP 1307 (2013) 118, arXiv:1305.1649. 
[13] S. Chang, S. K. Kang, J.-P. Lee, K. Y. Lee, S. C. Park, et al., Two Higgs doublet models for the LHC Higgs boson data at $\sqrt{s}=7$ and 8 TeV, JHEP 1409 (2014) 101, arXiv:1310.3374.

[14] K. Cheung, J. S. Lee, and P.-Y. Tseng, Higgcision in the Two-Higgs Doublet Models, JHEP 1401 (2014) 085, arXiv:1310.3937.

[15] A. Celis, V. Ilisie, and A. Pich, Towards a general analysis of LHC data within two-Higgs-doublet models, JHEP 1312 (2013) 095, arXiv:1310.7941].

[16] L. Wang and X.-F. Han, Status of the aligned two-Higgs-doublet model confronted with the Higgs data, JHEP 1404 (2014) 128, arXiv:1312.4759.

[17] J. Baglio, O. Eberhardt, U. Nierste, and M. Wiebusch, Benchmarks for Higgs Pair Production and Heavy Higgs Searches in the Two-Higgs-Doublet Model of Type II, Phys.Rev. D90 (2014) 015008, arXiv:1403.1264.

[18] S. Inoue, M. J. Ramsey-Musolf, and Y. Zhang, CPV Phenomenology of Flavor Conserving Two Higgs Doublet Models, Phys.Rev. D89 (2014) 115023, arXiv:1403.4257.

[19] N. Craig, J. Galloway, and S. Thomas, Searching for Signs of the Second Higgs Doublet, arXiv: 1305.2424

[20] V. Barger, L. L. Everett, H. E. Logan, and G. Shaughnessy, Scrutinizing the 125GeV Higgs boson in two Higgs doublet models at the LHC, ILC, and Muon Collider, Phys.Rev. D88 (2013) 115003, arXiv:1308.0052.

[21] M. Carena, I. Low, N. R. Shah, and C. E. Wagner, Impersonating the Standard Model Higgs Boson: Alignment without Decoupling, JHEP 1404 (2014) 015, arXiv:1310.2248.

[22] C.-Y. Chen, S. Dawson, and M. Sher, Heavy Higgs Searches and Constraints on Two Higgs Doublet Models, Phys.Rev. D88 (2013) 015018, arXiv:1305.1624.

[23] S. Kanemura, H. Yokoya, and Y.-J. Zheng, Complementarity in direct searches for additional Higgs bosons at the LHC and the International Linear Collider, Nucl.Phys. B886 (2014) 524-553, arXiv:1404.5835.

[24] L. Wang and X.-F. Han, Study of the heavy CP-even Higgs with mass 125 GeV in two-Higgs-doublet models at the LHC and ILC, JHEP 1411 (2014) 085, arXiv:1404.7437.

[25] G. Dorsch, S. Huber, and J. No, A strong electroweak phase transition in the 2HDM after LHC8, JHEP 1310 (2013) 029, arXiv:1305.6610. 
[26] J. Shu and Y. Zhang, Impact of a CP Violating Higgs Sector: From LHC to Baryogenesis, Phys.Rev.Lett. 111 (2013) 091801, arXiv:1304.0773.

[27] M. Ahmadvand, Baryogenesis within the two-Higgs-doublet model in the Electroweak scale, Int.J.Mod.Phys. A29 (2014) 1450090, arXiv:1308.3767.

[28] G. Dorsch, S. Huber, K. Mimasu, and J. No, Echoes of the Electroweak Phase Transition: Discovering a second Higgs doublet through $A_{0} \rightarrow Z H_{0}$, Phys.Rev.Lett. 113 (2014), no. 21 211802, $\operatorname{arXiv:1405.5537.}$

[29] A. Drozd, B. Grzadkowski, J. F. Gunion, and Y. Jiang, Two-Higgs-Doublet Models and Enhanced Rates for a 125 GeV Higgs, JHEP 1305 (2013) 072, arXiv:1211.3580].

[30] G. Belanger, B. Dumont, U. Ellwanger, J. Gunion, and S. Kraml, Global fit to Higgs signal strengths and couplings and implications for extended Higgs sectors, Phys.Rev. D88 (2013) 075008, arXiv:1306.2941.

[31] A. Arhrib, P. Ferreira, and R. Santos, Are There Hidden Scalars in LHC Higgs Results?, JHEP 1403 (2014) 053, arXiv:1311.1520.

[32] CMS Collaboration, Search for extended Higgs sectors in the $H \rightarrow$ hh and $A \rightarrow Z h$ channels in $\sqrt{s}=8 \mathrm{TeV}$ pp collisions with multileptons and photons final states, CMS-PAS-HIG-13-025, .

[33] D. Eriksson, J. Rathsman, and O. Stal, 2HDMC: Two-Higgs-Doublet Model Calculator Physics and Manual, Comput.Phys.Commun. 181 (2010) 189-205.

[34] D. Eriksson, J. Rathsman, and O. Stal, 2HDMC: Two-Higgs-doublet model calculator, Comput.Phys.Commun. 181 (2010) 833-834.

[35] CMS Collaboration, Higgs to tau tau (MSSM), CMS-PAS-HIG-13-021, .

[36] ATLAS Collaboration, Measurements of the properties of the Higgs-like boson in the four lepton decay channel with the ATLAS detector using 25 fb1 of proton-proton collision data, ATLAS-CONF-2013-013, .

[37] Properties of the Higgs-like boson in the decay $H \rightarrow Z Z \rightarrow 4 \ell$ in pp collisions at $\sqrt{s}=7$ and 8 TeV, Tech. Rep. CMS-PAS-HIG-13-002, CERN, Geneva, 2013.

[38] https://twiki.cern.ch/twiki/bin/view/CMSPublic/Hig13002TWiki\#Limit_Plot.

[39] Search for a heavy Higgs boson in the $H \rightarrow Z Z \rightarrow 2 \ell 2 n u$ channel in pp collisions at $\sqrt{s}=7$ and $8 \mathrm{TeV}$, Tech. Rep. CMS-PAS-HIG-13-014, CERN, Geneva, 2013. 
[40] A. Korytov. private communication.

[41] P. Ferreira, J. F. Gunion, H. E. Haber, and R. Santos, Probing wrong-sign Yukawa couplings at the LHC and a future linear collider, Phys.Rev. D89 (2014) 115003, arXiv:1403.4736].

[42] A. Efrati and Y. Nir, What if $\lambda_{h h h} \neq 3 m_{h}^{2} / v$, arXiv:1401.0935.

[43] S. Dawson, A. Gritsan, H. Logan, J. Qian, C. Tully, et al., Higgs Working Group Report of the Snowmass 2013 Community Planning Study, arXiv:1310.8361

[44] B. Coleppa, F. Kling, and S. Su, Exotic Decays Of A Heavy Neutral Higgs Through HZ/AZ Channel, JHEP 1409 (2014) 161, arXiv:1404.1922. 